Homology, Homotopy and Applications, vol.12(2), 2010, pp.39-108

\title{
THE HOMOTOPY THEORY OF STRONG HOMOTOPY ALGEBRAS AND BIALGEBRAS
}

\author{
J. P. PRIDHAM
}

\author{
(communicated by J. F. Jardine)
}

\begin{abstract}
Lada introduced strong homotopy algebras to describe the structures on a deformation retract of an algebra in topological spaces. However, there is no satisfactory general definition of a morphism of strong homotopy (s.h.) algebras. Given a monad T on a simplicial category $\mathcal{C}$, we instead show how s.h. $T$-algebras over $\mathcal{C}$ naturally form a Segal space. Given a distributive monadcomonad pair $(T, \perp)$, the same is true for s.h. $(T, \perp)$-bialgebras over $\mathcal{C}$; in particular this yields the homotopy theory of s.h. sheaves of s.h. rings. There are similar statements for quasimonads and quasi-comonads. We also show how the structures arising are related to derived connections on bundles.
\end{abstract}

\section{Introduction}

Given a monad $T$ acting on the category of topological spaces, Lada introduced (in $[\mathbf{C L M}]$ ) the notion of a strong homotopy (s.h.) T-algebra. This characterises the structures arising on deformation retracts of $T$-algebras. Indeed, when $T$ is an operad, there is a bar construction which realises every s.h. T-algebra as such a deformation retract. The formulation of s.h. algebras does not use any special properties of topological spaces, so it adapts to any simplicial category and likewise adapts to describe s.h. coalgebras of a comonad $\perp$.

Structures such as Hopf algebras or sheaves of rings cannot be described as algebras of a monad $T$ or as coalgebras of a comonad $\perp$. However, in both cases, there are both a natural monad $T$ (governing the algebraic structure) and a comonad $\perp$ (governing the coalgebraic structure), satisfying a distributivity condition. This seems to have first been described by Van Osdol in [VO] in order to develop bicohomology theory. Independent rediscoveries have appeared in $[\mathbf{B J}]$, [PW, Appendix C] and [Pri2, §2]. This permits the characterisation of a compatibility condition for the algebraic and coalgebraic structures.

In [Pri5], it was observed that the equations defining a s.h. T-algebra can also be used to define s.h. $(T, \perp)$-bialgebras associated to a distributive monad-comonad

This work was supported by Trinity College, Cambridge and by the Engineering and Physical Sciences Research Council [grant number EP/F043570/1].

Received August 9, 2009, revised March 15, 2010, April 6, 2010; published on July 19, 2010.

2000 Mathematics Subject Classification: 18C15; 55U40, 18D20, 18 G30.

Key words and phrases: algebraic theories, simplicial categories, Segal spaces.

This article is available at http://intlpress.com/HHA/v12/n2/a3

Copyright (c) 2010, International Press. Permission to copy for private use granted. 
pair $(T, \perp)$. In particular, this gives rise to a notion of s.h. sheaves of s.h. algebras (on any site with enough points), yielding important applications in algebro-geometric deformation theory.

The first main result in this paper is Proposition 2.9, which provides a single unified framework for dealing with algebras, coalgebras and bialgebras. This then combines with Proposition 5.7 and Corollaries 5.19 and 5.25 to give three possible models for the $\infty$-category of s.h. algebras, coalgebras and bialgebras. These models are shown to be equivalent in Propositions 5.15 and 5.24. Finally, Theorem 6.23 shows how this $\infty$-category is related to the Maurer-Cartan functor featuring in [Man2] and [Hin].

These results have applications in derived deformation theory, which starts with a moduli functor from algebras to groupoids and seeks a derived moduli functor from simplicial algebras to $\infty$-groupoids. By describing deformation problems in terms of bialgebraic structures, $[$ Pri5] and [Pri4] apply the results of this paper to construct derived deformation functors, and these are being extended to (global) derived moduli functors in [Pri6]. Even where there are other possible approaches to defining derived moduli functors, strong homotopy bialgebras often provide a more concrete description, and have the crucial property that the functor is left exact, making it easier to verify Lurie's representability theorem ([Lur $]$ ). For some examples, such as Hopf algebras, strong homotopy bialgebras provide the only known means of constructing derived deformations.

A major failing of the theory of s.h. algebras is that there is no satisfactory general definition of morphisms. In $[\mathbf{C L M}]$, this difficulty was obviated by considering morphisms of the associated bar constructions, but this has several disadvantages. For applications in deformation theory, the main problem is that the bar construction does not respect finite limits, in general. For bialgebras, the difficulty is even more fundamental, since the bar and cobar constructions will, in general, be incompatible, so the s.h. structures cannot be rectified. This is essentially the phenomenon that a lax sheaf of lax simplicial rings cannot be replaced by a strict sheaf of strict simplicial rings.

Rather than seeking to define a simplicial category of s.h. algebras or bialgebras explicitly, we instead construct Segal spaces. This is a special type of simplicial space (i.e., bisimplicial set) introduced by Rezk in $[\mathbf{R e z}]$ as a model for homotopy theories, and Bergner showed, in [Ber2], that the associated model category is Quillenequivalent to the model category of simplicial categories, so any Segal space naturally gives rise to a simplicial category.

Our approach makes use of a generalisation of the theory of homotopy monoids expounded by Leinster in [Lei2]. We introduce a slight generalisation, the quasicomonoid, of a homotopy comonoid and associate a simplicial set $\mathrm{MC}(E)$, the MaurerCartan space, to any simplicial quasi-comonoid $E$. When $E^{0}$ is a group rather than a monoid, a more natural object is the Deligne space $\operatorname{Del}(E)$, which is the homotopy quotient of $\underline{\mathrm{MC}}(E)$ by $E^{0}$. We also develop the concept of a quasi-descent datum; put simply, a quasi-descent datum is to a quasi-comonoid as a category is to a monoid.

Proposition 2.12 then shows how a distributive monad-comonad pair on a simplicial category $\mathcal{C}$ naturally enriches it to a simplicial quasi-descent datum, with $\underline{\mathrm{MC}}$ evaluated at any object $x$ of $\mathcal{C}$ giving the space of s.h. bialgebras over $x$. Moreover, there is a natural quasi-comonoid associated to any diagram in $\mathcal{C}$, and we use this to 
define a simplicial space $\mathcal{M C}$ by mimicking the nerve construction (Definition 3.25). In Proposition 5.7 this is seen to be a Segal space.

Another source of quasi-comonoids is from cosimplicial groups, with the construction $\mathcal{E}$ given in Definition 6.6. In this case, the Maurer-Cartan space admits a simpler description (Proposition 6.8). If $X$ is a simplicial set, $G$ a simplicial group and $\mathrm{C}^{\bullet}(X, G)$ the cosimplicial simplicial group of $G$-cochains on $X$, then $\operatorname{Del}\left(\mathcal{E} \mathrm{C}^{\bullet}(X, G)\right)$ is equivalent to $\operatorname{Hom}(X, \bar{W} G)$, where $\bar{W} G$ is the classifying space of $G$ (Proposition 6.11 and Remark 6.10). If the cosimplicial group comes from denormalising and exponentiating a differential graded Lie algebra, then the Maurer-Cartan space is equivalent to the classical Maurer-Cartan space of derived connections, given by the equation

$$
d \omega+\frac{1}{2}[\omega, \omega]=0,
$$

for $\omega$ of total degree 1 (Corollary 6.27).

The structure of the paper is as follows.

In Section 1, we recall Leinster's comonoids up to homotopy, and introduce quasicomonoids and then the category $Q D$ Dat of quasi-descent data. There is an adjunction (Lemma 1.15):

$$
\text { Cat } \stackrel{\text { alg* }_{<}}{\stackrel{\perp}{\perp}} \text { QDat. }
$$

In Section 2, we introduce monads and comonads and show how a monad $T$ on a category $\mathcal{C}$ naturally enriches it to a quasi-descent datum $\mathcal{D}(\mathcal{C}, T)$. This has the properties that alg $\mathcal{D}(\mathcal{C}, \top)$ is the category $\mathcal{C}^{\top}$ of $\top$-algebras, and that alg ${ }^{*} \mathcal{C}=\mathcal{D}(\mathcal{C}$, id $)$. There is a similar result for $(\top, \perp)$-bialgebras.

Section 3 begins the work of extending these constructions to simplicial categories. Distributive monad-comonad pairs on simplicial categories give rise to simplicial quasidescent data, but the functor alg above destroys higher order information. We therefore begin (Definition 3.5) by defining the higher Maurer-Cartan functor $\underline{\mathrm{MC}}$ from simplicial quasi-comonoids $s Q M^{*}$ to simplicial sets $\mathbb{S}$. Given an object $x$ of a simplicial quasi-descent datum $\mathcal{D}$, there is a simplicial quasi-comonoid $\mathcal{D}(x, x)$, and the vertices of $\underline{\mathrm{MC}}(\mathcal{D}(x, x))$ correspond closely to Lada's set of strong homotopy T-algebras over $x$, when $\mathcal{D}=\mathcal{D}(\mathcal{C}, T)$ (Remark 3.13). We then extend this to a simplicial space (i.e. a bisimplicial set) $\mathcal{M C}(\mathcal{D})$ (Definition 3.25), which mimics the nerve construction by developing constructions loosely corresponding to strong homotopy diagrams of strong homotopy algebras. When the underlying category is a groupoid, $\mathcal{D} \mathcal{E} \mathcal{L}(\mathcal{D})$ is the homotopy quotient of $\mathcal{M C}(\mathcal{D})$ by morphisms in $\mathcal{D}$, and Del is similarly related to MC.

Section 4 is primarily concerned with the study of quasi-comonoids in $(\mathrm{Ab}, \times)$ (abelian groups with the monoidal structure $\times$ ). These are equivalent to cosimplicial abelian groups (Lemma 4.1), and we exploit this to develop cohomology of quasicomonoids, and relate it to homotopy groups of $\underline{\mathrm{MC}}$ and Del. We also study nerves $B \Gamma$ of quasi-comonoids in groupoids, in order to understand fundamental groupoids of simplicial quasi-comonoids. We then introduce linear quasi-comonoids (i.e. quasicomonoids in $(\mathrm{Ab}, \otimes))$. These allow us to relate cohomology of a simplicial quasicomonoid to homology of the underlying simplicial abelian group (Proposition 4.22). 
This gives a cohomological characterisation of when a cofibrant simply connected simplicial quasi-comonoid is contractible (Corollary 4.23). These results are then extended to quasi-bicomonoids, which play a crucial role in the construction of $\mathcal{M C}$.

Although the functor alg above is poorly suited to simplicial categories, its left adjoint alg* extends naturally and preserves weak equivalences. There is a model structure on $s Q D a t$, and the purpose of Section 5 is to relate, for $\mathcal{D}$ fibrant, the simplicial spaces $\mathcal{M C}(\mathcal{D})$ and $\mathcal{D} \mathcal{E} \mathcal{L}(\mathcal{D})$ with the simplicial space $\mathcal{N}(\mathcal{D})$ given by the derived function complexes

$$
\mathcal{N}(\mathcal{D})_{n}=\operatorname{Map}_{s Q \text { Dat }}^{h}\left(\operatorname{alg}^{*} \mathbf{n}, \mathcal{D}\right),
$$

where $\mathbf{n}$ is the category associated to the poset $[0, n]$. When $\mathcal{D}$ is constructed from a distributive monad-comonad pair on a simplicial category $\mathcal{B}$, we think of $\mathcal{M C}(\mathcal{D})$, $\mathcal{D E} \mathcal{L}(\mathcal{D})$ and $\mathcal{N}(\mathcal{D})$ as candidates for the simplicial space of s.h. bialgebras over $\mathcal{B}$.

It turns out that $\mathcal{M C}(\mathcal{D})$ is a Segal space, while $\mathcal{N}(\mathcal{D})$ and $\mathcal{D E} \mathcal{L}(\mathcal{D})$ are complete Segal spaces (Proposition 5.7, Corollaries 5.19 and 5.25). There are Dwyer-Kan equivalences between these Segal spaces (Propositions 5.15 and 5.24), so they are all weakly equivalent in the complete Segal space model structure from [Rez]. Moreover, Theorem 5.21 shows how $\mathcal{M C}$ and $\mathcal{N}$ can both be regarded as the derived right adjoint of alg*, even though alg* is not left Quillen.

Section 6 establishes a simpler Maurer-Cartan space construction for cosimplicial simplicial groups, yielding the equivalence $\operatorname{Del}\left(\mathcal{E} \mathrm{C}^{\bullet}(X, G)\right) \simeq \underline{\operatorname{Hom}}(X, \bar{W} G)$ described earlier, and simplifies this description further when $G$ is a formal Lie group, via Theorem 6.23 .

The appendices establish a framework for applying these results more widely. In Appendix A, quasi-monads and quasi-comonads are introduced, motivated by the need for an analogue of the homotopy operads of [ $\mathbf{v d L}$. We show what it would mean for a quasi-monad to distribute over a quasi-comonad, while ensuring that these weaker structures still enhance a category to form a quasi-descent datum. In Appendix B, we relate $A_{\infty}$-algebras to quasi-semigroups, and thus compare quasi-operads with homotopy operads.

\section{Acknowledgements}

I would like to thank Carlos Simpson for alerting me to the similarities between the SDCs of [Pri1] and Leinster's homotopy monoids ([Lei2]). I would also like to thank the anonymous referee heartily for diligently identifying many errors and omissions in the original manuscript.

\section{Contents}

1 Monoidal structures up to homotopy $\quad 43$

1.1 Quasi-comonoids .................... 44

1.2 Quasi-descent data ................... . . . . . . . . . . . . . . . . . . . . . . . . 45

1.3 Adjoint functors . . . . . . . . . . . . . . . . . . . . . . . . . . . . . . . . 47

1.4 Quasi-bicomonoids and diagonals . . . . . . . . . . . . . . . 48

1.5 Nerves . . . . . . . . . . . . . . . . . . . . 48 
2 Algebras, coalgebras and bialgebras $\quad \mathbf{5 2}$

2.1 Algebras and coalgebras . . . . . . . . . . . . . . . . 52

2.2 Quasi-descent data from monads . . . . . . . . . . . . . . . 53

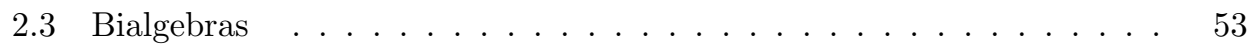

3 Simplicial structures $\quad 55$

3.1 Simplicial quasi-comonoids . . . . . . . . . . . . . . . . 55

3.2 Simplicial categories . . . . . . . . . . . . . . . . . . . 59

3.3 Simplicial quasi-descent data . . . . . . . . . . . . . . . . 59

3.4 Simplicial bicomonoids . . . . . . . . . . . . . . . . . . . 62

3.5 Nerves . . . . . . . . . . . . . . . . . . . . . . . . 62

4 Abelian groups and cohomology $\quad 66$

4.1 Cosimplicial abelian groups . . . . . . . . . . . . . . . . 66

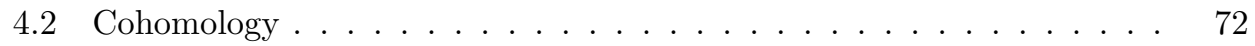

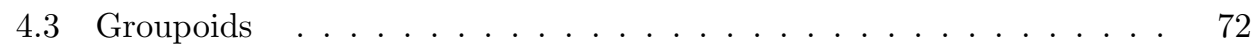

4.4 Linear quasi-comonoids . . . . . . . . . . . . . . . . . . 73

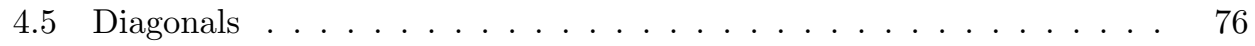

5 Mapping spaces $\quad 79$

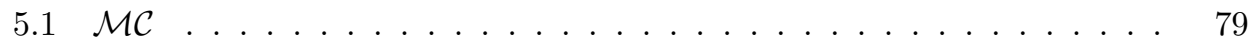

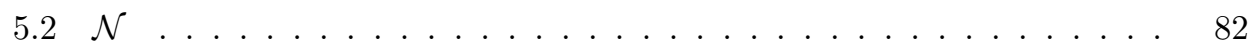

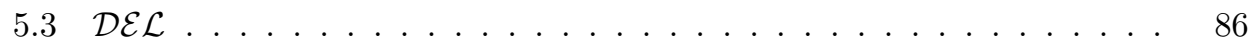

6 Maurer-Cartan and classifying spaces $\quad 88$

6.1 Groups. . . . . . . . . . . . . . . . . . . 88

6.2 Lie algebras . . . . . . . . . . . . . . . . . . . . . 93

A Quasi-monads, quasi-comonads and quasi-distributivity $\quad 98$

A.1 Quasi-monads and quasi-comonads . . . . . . . . . . . . . . . 98

A.2 Distributivity for quasi-monads and quasi-comonads . . . . . . . 100

B $A_{\infty}$-algebras and homotopy operads $\quad 101$

B.1 Homotopy semigroups and semicogroups . . . . . . . . . . . . . . . . 101

B.2 Homotopy semigroups in abelian categories . . . . . . . . . . . . 102

B.3 DG co-operads . . . . . . . . . . . . . . . . . . . . . . . . 105

\section{Monoidal structures up to homotopy}

In this section, we will introduce various structures which will provide the framework for the rest of the paper. The main concepts are those of a quasi-comonoids and quasidescent data, which will provide the intermediate step between monads and comonads on one hand, and strong homotopy structures on the other. 


\subsection{Quasi-comonoids}

Definition 1.1. Define $\Delta_{* *}$ to be the subcategory of the ordinal number category $\Delta$ containing only those non-decreasing (i.e. $f(i+1) \geqslant f(i)$ ) morphisms $f: \mathbf{m} \rightarrow \mathbf{n}$ with $f(0)=0, f(m)=n$. We define a monoidal structure on this category by setting $\mathbf{m} \otimes \mathbf{n}=\mathbf{m}+\mathbf{n}$, with

$$
(f \otimes g)(i)= \begin{cases}f(i) & i \leqslant m \\ g(i-m)+p & i \geqslant m\end{cases}
$$

for $f: \mathbf{m} \rightarrow \mathbf{p}, g: \mathbf{n} \rightarrow \mathbf{q}$.

Remark 1.2. There is an isomorphism $\Delta_{* *}^{\mathrm{opp}} \cong \Delta_{0}$, the category of finite sets (i.e. $\Delta \sqcup \emptyset)$ given by $\mathbf{n} \mapsto \mathbf{n}-\mathbf{1}$, where $-\mathbf{1}:=\emptyset$, with the coboundary morphisms $\partial^{i}$ mapping to $\sigma_{i-1}$ and the coface morphisms $\sigma^{i}$ mapping to $\partial_{i}$.

Remark 1.3. Given a category $\mathcal{C}$, a functor $X: \Delta_{* *} \rightarrow \mathcal{C}$ consists of objects $X^{n} \in \mathcal{C}$, with all of the operations $\partial^{i}, \sigma^{i}$ of a cosimplicial complex except $\partial^{0}, \partial^{n+1}: X^{n} \rightarrow X^{n+1}$.

Definition 1.4. Given a monoidal category $\mathcal{C}$, define a quasi-comonoid $X$ in $\mathcal{C}$ to be a lax monoidal functor $X: \Delta_{* *} \rightarrow \mathcal{C}$. This means that we have maps $\zeta^{m n}: X^{m} \otimes X^{n} \rightarrow$ $X^{m+n}, \zeta^{0}: 1 \rightarrow X^{0}$, satisfying naturality and coherence, where 1 is the unit in the category. If $\mathcal{C}$ is a model category, then we say that $X$ is a homotopy comonoid whenever the maps $\zeta^{m n}, \zeta^{0}$ are all weak equivalences. This is equivalent to the definition in [Lei2], via the comparison of Remark 1.2.

Define a quasi-monoid in $\mathcal{C}$ to be a quasi-comonoid in $\mathcal{C}^{\text {opp }}$. We let $Q M^{*}(\mathcal{C})$ denote the category of quasi-comonoids in $\mathcal{C}$.

Lemma 1.5. Giving a quasi-comonoid $X$ in $\mathcal{C}$ is equivalent to giving objects $X^{n} \in \mathcal{C}$ for $n \in \mathbb{N}_{0}$, together with morphisms

$$
\begin{array}{ll}
\partial^{i}: X^{n} \rightarrow X^{n+1} & 1 \leqslant i \leqslant n, \\
\sigma^{i}: X^{n} \rightarrow X^{n-1} & 0 \leqslant i<n,
\end{array}
$$

an associative product $\zeta^{m n}: X^{m} \otimes X^{n} \rightarrow X^{m+n}$, with identity $\zeta^{0}: 1 \rightarrow X^{0}$, where 1 is the unit in the category, satisfying:

1. $\partial^{j} \partial^{i}=\partial^{i} \partial^{j-1} \quad i<j$.

2. $\sigma^{j} \sigma^{i}=\sigma^{i} \sigma^{j+1} \quad i \leqslant j$.

3. $\sigma^{j} \partial^{i}= \begin{cases}\partial^{i} \sigma^{j-1} & i \leqslant j \\ \operatorname{id} & i=j, i=j+1 \\ \partial^{i-1} \sigma^{j} & i \geqslant j+1 .\end{cases}$

4. $\zeta^{m+1, n}\left(\partial^{i} \otimes \mathrm{id}\right)=\partial^{i} \zeta^{m n}$.

5. $\zeta^{m, n+1}\left(\mathrm{id} \otimes \partial^{i}\right)=\partial^{i+m} \zeta^{m n}$.

6. $\zeta^{m-1, n}\left(\sigma^{i} \otimes \mathrm{id}\right)=\sigma^{i} \zeta^{m n}$.

7. $\zeta^{m, n-1}\left(\mathrm{id} \otimes \sigma^{i}\right)=\sigma^{i+m} \zeta^{m n}$.

Proof. This is a straightforward consequence of Remark 1.3, together with an analysis of the interaction in $\Delta_{* *}$ of the monoidal structure and the morphisms $\sigma^{i}, \partial^{i}$. 
Remark 1.6. When the maps $\zeta^{m n}, \zeta^{0}$ are all isomorphisms, this becomes equivalent to the definition of a comonoid $C$. The correspondence is given by setting $X^{n}:=C^{\otimes n}$ and letting $\partial^{1}: C \rightarrow C \otimes C$ be the coproduct.

In order to simplify the notation, we will write $x * y$ instead of $\zeta^{m, n}(x, y)$ from now on.

\subsubsection{Maurer-Cartan}

Observe that the category of comonoids in $($ Set, $\times$ ) is equivalent to Set itself, since comultiplication $\Delta: X \rightarrow X \times X$ is necessarily the diagonal embedding. This gives a functor $\iota$ : Set $\rightarrow Q M^{*}($ Set) from sets to quasi-comonoids.

Definition 1.7. Define the functor MC: $Q M^{*}($ Set $) \rightarrow$ Set by

$$
\operatorname{MC}(E):=\operatorname{Hom}(\iota \bullet, E),
$$

where $\bullet$ is the one-point set. Explicitly,

$$
\operatorname{MC}(E)=\left\{\omega \in E^{1}: \sigma^{0} \omega=1, \partial^{1} \omega=\omega * \omega\right\} .
$$

The reason for this notation is that any cosimplicial unital ring $R$ has a quasicomonoid structure, with $*$ given by the Alexander-Whitney cup product $U$, and then

$$
\begin{aligned}
\operatorname{MC}(R) & =\left\{1_{1}+\alpha \in R^{1}: \sigma^{0} \alpha=0,1_{2}+\partial^{1} \alpha=1_{2}+1_{1} \cup \alpha+\alpha \cup 1_{1}+\alpha \cup \alpha\right\} \\
& =1+\left\{\alpha \in N^{1} R: \partial^{1} \alpha=\partial^{2} \alpha+\partial^{0} \alpha+\alpha \cup \alpha\right\} \\
& =1+\left\{\alpha \in N^{1} R: d \alpha+\alpha \cup \alpha=0\right\}
\end{aligned}
$$

where $N R$ is the cosimplicial normalisation of $R$, with differential $d$. Thus $\operatorname{MC}(R)$ is just the classical set of Maurer-Cartan forms of the differential graded algebra $N R$, which parametrises flat connections on a vector bundle.

\subsection{Quasi-descent data}

We will now introduce quasi-descent data, which will form the bridge between categories equipped with monads and/or comonads, and Segal spaces. Section 2 will show how monads and comonads give rise to quasi-descent data, while much of the remainder of the paper relates quasi-descent data to Segal spaces.

Definition 1.8. Given a monoidal category $\mathcal{C}$ and a set $\mathcal{O}$, let a $\mathcal{C}$-valued descent datum $D=(C, G)$ on objects $\mathcal{O}$ consist of:

1. objects $G(a, b) \in \mathcal{C}$ for each pair $a, b \in \mathcal{O}$,

2. compatible systems $G(a, b) \otimes G(b, c) \cong G(a, c)$ and $G(a, a) \cong 1$ of transition isomorphisms, for all $a, b, c \in \mathcal{O}$ (the cocycle condition),

3. comonoids $C(a) \in \mathcal{C}$ for each $a \in \mathcal{O}$,

4. isomorphisms $C(a) \otimes G(a, b) \cong G(a, b) \otimes C(b)$ for all $a, b \in \mathcal{O}$, compatible with the comonoidal structures and transition isomorphisms.

Note that these conditions imply that $G(b, a) \otimes C(a) \otimes G(a, b)$ is isomorphic as a comonoid to $C(b)$. 
The reason for this terminology is that for an open cover $\left\{U_{a}\right\}_{a \in \mathcal{O}}$ of topological spaces $X$, a descent datum consists of sheaves $C_{a}$ on $U_{a}$, with isomorphisms $g_{a b}:\left.\left.C_{b}\right|_{U_{a} \cap U_{b}} \rightarrow C_{a}\right|_{U_{a} \cap U_{b}}$, with $g_{a b} \circ g_{b c}=g_{a c}:\left.\left.C_{c}\right|_{U_{a} \cap U_{b} \cap U_{c}} \rightarrow C_{a}\right|_{U_{a} \cap U_{b} \cap U_{c}}$ (and $g_{a a}$ necessarily the identity).

Definition 1.9. Let $\operatorname{Dat}_{\mathcal{O}}(\mathcal{C})$ be the category of $\mathcal{C}$-valued descent data on objects $\mathcal{O}$, and let $\operatorname{Dat}(\mathcal{C})$ be the category of pairs $(\mathcal{O}, D)$, for $\mathcal{O}$ a set and $D=(C, G)$ a descent datum on objects $\mathcal{O}$. Write Dat $:=\operatorname{Dat}($ Set,$\times)$ and define $\operatorname{Dat}_{\mathcal{O}}$ similarly. Note that there is a functor $\operatorname{Dat}(\mathcal{C}) \rightarrow \operatorname{Cat}(\mathcal{C})$ to $\mathcal{C}$-enriched categories, sending $(\mathcal{O}, D)$ to the category with objects $\mathcal{O}$ and morphisms $G(x, y)$.

Definition 1.10. Given a monoidal category $\mathcal{C}$ and a set $\mathcal{O}$, let a $\mathcal{C}$-valued quasidescent datum on objects $\mathcal{O}$ consist of:

1. objects $X(a, b) \in \mathcal{C}^{\Delta_{* *}}$ for all $a, b \in \mathcal{O}$,

2. morphisms $X(a, b)^{m} \otimes X(b, c)^{n} \stackrel{*}{\rightarrow} X(a, c)^{m+n}$ making the following diagram commute for all $a, b, c \in \mathcal{O}$

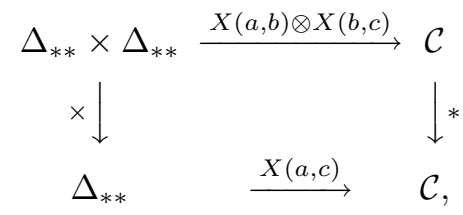

3. morphisms $1 \rightarrow X(a, a)^{0}$ for all $a \in \mathcal{O}$, acting as the identity for the multiplication $*$.

Remarks 1.11. A $\mathcal{C}$-valued (quasi-)descent datum on the set with one object is just a (quasi-)comonoid in $\mathcal{C}$. Given a $\mathcal{C}$-valued descent datum $(C, G)$ on objects $\mathcal{O}$, we may define a quasi-descent datum $X$ by $X(a, b)^{n}:=C(a)^{\otimes n} \otimes G(a, b) \cong G(a, b) \otimes C(b)^{\otimes n}$ via the transition maps, with the maps $\partial^{i}$ given by the coproducts on $C$.

If the category $\mathcal{C}$ contains all finite coproducts, then we may define a monoidal structure on $\mathcal{C}^{\Delta_{* *}}$ by setting

$$
(X \otimes Y)^{n}:=\coprod_{a+b=n} X^{a} \otimes Y^{b},
$$

with operations given by

$$
\begin{array}{r}
\partial^{i}(x \otimes y)= \begin{cases}\left(\partial^{i} x\right) \otimes y & i \leqslant a \\
x \otimes\left(\partial^{i-a} y\right) & i>a,\end{cases} \\
\sigma^{i}(x \otimes y)= \begin{cases}\left(\sigma^{i} x\right) \otimes y & i<a, \\
x \otimes\left(\sigma^{i-a} y\right) & i \geqslant a .\end{cases}
\end{array}
$$

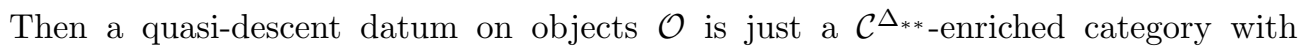
objects $\mathcal{O}$.

Definition 1.12. Let $Q \operatorname{Dat}(\mathcal{C})$ be the category of $\mathcal{C}$-valued quasi-descent data, i.e. of pairs $(\mathcal{O}, X)$ for $\mathcal{O}$ a set and $X$ a quasi-descent datum on objects $\mathcal{O}$. This admits a functor to the category of $\mathcal{C}$-enriched categories by taking the Hom-space underlying $X(a, b)$ to be $X(a, b)^{0}$. Let $Q$ Dat $:=Q \operatorname{Dat}($ Set, $\times)$ and $s Q \operatorname{Dat}:=Q \operatorname{Dat}(\mathbb{S}, \times)$. 
Let $Q D p d$ (resp. $s Q D p d$ ) be the full subcategory of $Q D a t$ (resp. $s Q D a t$ ) consisting of objects whose underlying categories (resp. simplicial categories) are groupoids (resp. simplicial groupoids). In other words, all elements of $X(a, b)^{0}$ must be invertible under $*$.

\subsection{Adjoint functors}

From now on, we will systematically make use of the identification in Remarks 1.11

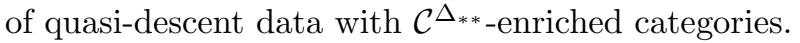

We have seen that there is a functor $(-)^{0}: Q$ Dat $\rightarrow$ Cat given by sending $\mathcal{D}$ to the category $\mathcal{D}^{0}$ with objects $\mathrm{Ob} \mathcal{D}^{0}:=\mathrm{Ob} \mathcal{D}$ and morphisms $\mathcal{D}^{0}(a, b):=\mathcal{D}(a, b)^{0}$.

Lemma 1.13. The functor $(-)^{0}$ has a right adjoint $\mathrm{alg}^{*}$, given by $\mathrm{Ob} \lg ^{*} \mathcal{C}=\mathrm{Ob} \mathcal{C}$, with $\left(\operatorname{alg}^{*} \mathcal{C}\right)(a, b)^{n}=\mathcal{C}(a, b)$ for all $n$, with multiplication as in $\mathcal{C}$, and all operations $\partial^{i}, \sigma^{i}$ acting trivially.

Proof. Given a functor $F: \mathcal{D}^{0} \rightarrow \mathcal{C}$, the associated morphism $\mathcal{D} \rightarrow \operatorname{alg}^{*} \mathcal{C}$ in $Q$ Dat is given by $F$ on objects, with morphisms

$$
F \circ\left(\sigma^{0}\right)^{n}: \mathcal{D}(a, b)^{n} \rightarrow \mathcal{C}(a, b)=\left(\operatorname{alg}^{*} \mathcal{C}\right)(a, b)^{n} .
$$

That all morphisms $\mathcal{D} \rightarrow \operatorname{alg}^{*} \mathcal{C}$ arise in this way follows because $\sigma^{0}$ acts trivially on $\left(\operatorname{alg}^{*} \mathcal{C}\right)(a, b)$.

Definition 1.14. Define alg: $Q$ Dat $\rightarrow$ Cat as follows: The objects of $\operatorname{alg}(\mathcal{D})$ are pairs $(D, \omega)$, for $D \in \mathrm{Ob} \mathcal{D}$ and $\omega \in \mathrm{MC}(\mathcal{D}(D, D))$, for $\mathrm{MC}$ as in Definition 1.7. Morphisms from $(D, \omega)$ to $\left(D^{\prime}, \omega^{\prime}\right)$ are given by $f \in \mathcal{D}\left(D, D^{\prime}\right)^{0}$ such that

$$
f * \omega=\omega^{\prime} * f \in \mathcal{D}\left(D, D^{\prime}\right)^{1} .
$$

Lemma 1.15. The functor $\operatorname{alg}^{*}$ is left adjoint to alg.

Proof. Given a functor $F: \mathcal{C} \rightarrow \operatorname{alg}(\mathcal{D})$, we construct the corresponding morphism $G:$ alg $^{*} \mathcal{C} \rightarrow \mathcal{D}$ as follows: For $a \in \mathrm{Ob} C$, set $G(a) \in \mathrm{Ob} D$ to be the object underlying $F(a)$, and then define $G: \operatorname{alg}^{*} \mathcal{C}(a, b)^{n} \rightarrow \mathcal{D}(G a, G b)^{n}$ by

$$
G(x):=\omega_{F(b)}^{n} * F(x)=F(x) * \omega_{F(a)}^{n},
$$

where $F(a)=\left(G(a), \omega_{F(a)}\right)$. Conversely, any morphism $G$ : $\operatorname{alg}^{*} \mathcal{C} \rightarrow \mathcal{D}$ is determined by $\operatorname{alg}^{*} \mathcal{C}^{0} \rightarrow \mathcal{D}^{0}$, together with the elements $G\left(\operatorname{id}_{a}\right) \in \mathcal{D}(a, a)^{1}$.

Remark 1.16. Note that the functor alg* is fully faithful, so $\left(\operatorname{alg}^{*} \mathcal{C}\right)^{0} \simeq \mathcal{C} \simeq \operatorname{alg}$ alg* $\mathcal{C}$.

Lemma 1.17. The functor $(-)^{0}:$ QDat $\rightarrow$ Cat has a left adjoint.

Proof. The left adjoint is the functor $(\mathrm{id} / \emptyset)$ given by $\mathrm{Ob}(\mathrm{id} / \emptyset)(\mathcal{C})=$ Ob $\mathcal{C}$, with morphisms

$$
(\mathrm{id} / \emptyset)(\mathcal{C})(a, b)^{n}= \begin{cases}\mathcal{C}(a, b) & n=0 \\ \emptyset & n>0\end{cases}
$$




\subsection{Quasi-bicomonoids and diagonals}

Definition 1.18. Given a monoidal category $\mathcal{C}$, let the category $Q M M^{*}(\mathcal{C})$ of quasibicomonoids consist of lax monoidal functors $X: \Delta_{* *} \times \Delta_{* *} \rightarrow \mathcal{C}$.

Definition 1.19. Define a functor diag: $Q M M^{*}(\mathcal{C}) \rightarrow Q M^{*}(\mathcal{C})$ via the diagonal functor $\Delta_{* *} \rightarrow \Delta_{* *} \times \Delta_{* *}$. Explicitly, $(\operatorname{diag} E)^{n}=E^{n n}$, with the same product and identity as $E$ and operations $\partial^{i}=\partial_{h}^{i} \partial_{v}^{i}$ and $\sigma^{i}=\sigma_{h}^{i} \sigma_{v}^{i}$.

The functor diag preserves all limits, so by the Special Adjoint Functor Theorem ([Mac, Theorem V.8.2]), it will have a left adjoint diag* for all the categories $\mathcal{C}$ which we will encounter, since they all satisfy the solution set condition.

Definition 1.20. The category of quasi-comonoids in Set $\times$ Set is isomorphic to Set $\times$ Set, with comultiplication necessarily given by the diagonal

$$
(X, Y) \rightarrow(X \times X, Y \times Y) .
$$

Inclusion of comonoids in quasi-comonoids then gives a functor

$$
\iota: \text { Set } \times \text { Set } \rightarrow Q M M^{*}(\text { Set }),
$$

with $\iota(X, Y)^{m, n}=X^{m} \times Y^{n}$.

Lemma 1.21. For $E^{\bullet \bullet \bullet} \in Q M M^{*}($ Set $)$,

$$
\operatorname{MC}(\operatorname{diag} E) \cong\left\{(\alpha, \beta) \in \operatorname{MC}\left(E^{\bullet, 0}\right) \times \operatorname{MC}\left(E^{0, \bullet}\right): \alpha * \beta=\beta * \alpha \in E^{11}\right\} .
$$

Proof. Given $\omega \in \mathrm{MC}(\operatorname{diag} E)$, set $\alpha:=\sigma_{v}^{0} \omega, \beta:=\sigma_{h}^{0} \omega$. Since $\sigma^{0} \omega=1$, we have $\sigma_{h}^{0} \alpha=$ $\sigma_{v}^{0} \beta=1$. Applying the operations $\left(\sigma_{v}^{0}\right)^{2}, \sigma_{h}^{1} \sigma_{v}^{0}, \sigma_{h}^{1} \sigma_{v}^{0},\left(\sigma_{h}^{0}\right)^{2}$ (respectively) to the equation $\omega * \omega=\partial^{1} \omega$ gives the equations

$$
\alpha * \alpha=\partial_{h}^{1} \alpha, \quad \alpha * \beta=\omega, \quad \beta * \alpha=\omega, \quad \beta * \beta=\partial_{v}^{1} \beta .
$$

This shows that the function $\omega \mapsto(\alpha, \beta)$ is well-defined on the sets above and has inverse $(\alpha, \beta) \mapsto \alpha * \beta$.

Corollary 1.22. diag $^{*} \iota \bullet=\iota(\bullet, \bullet)$.

Proof. Given a morphism $f: \iota(\bullet, \bullet) \rightarrow E$ in $Q M M^{*}$ (Set), let $\alpha, \beta$ be the images of the unique elements of $\iota(\bullet, \bullet)^{1,0}, \iota(\bullet, \bullet)^{0,1}$. These generate $\iota(\bullet, \bullet)$, subject to the conditions of Lemma 1.21.

\subsection{Nerves}

We will now see how to associate quasi-comonoids to small diagrams. In Remark 3.27 this will give rise to the notion of strong homotopy diagrams.

\subsubsection{Categories}

Given a set $\mathcal{O}$, we will write $\operatorname{Cat}_{\mathcal{O}}$ for the category of categories with object set $\mathcal{O}$. 
Definition 1.23. Given $K \in \mathbb{S}$, define $P_{K}:$ Cat $_{K_{0}} \rightarrow Q M^{*}($ Set $)$ by

$$
P_{K}(\mathcal{C})^{a}=\prod_{x \in K_{a}} \mathcal{C}\left(\left(\partial_{0}\right)^{a} x,\left(\partial_{1}\right)^{a} x\right)
$$

with operations

$$
\begin{aligned}
\partial^{i}(e)(x) & :=e\left(\partial_{i} x\right), \\
\sigma^{j}(e) & :=e\left(\sigma_{j} y\right), \\
(f * e)(z) & :=f\left(\left(\partial_{a+1}\right)^{b} z\right) \circ e\left(\left(\partial_{0}\right)^{a} z\right),
\end{aligned}
$$

for $f \in P_{K}(\mathcal{C})^{a}, e \in P_{K}(\mathcal{C})^{b}$.

Let $P_{n}:=P_{\Delta^{n}}$.

Let $\mathrm{Cat}_{n}$ be the category of categories on (the $\left.n+1\right)$ objects $[0, n]$.

Lemma 1.24. The functor $P_{n}:$ Cat $_{n} \rightarrow Q M^{*}($ Set $)$ has a left adjoint $P_{n}^{*}$, given by

$$
\left(P_{n}^{*} E\right)(i, j)= \begin{cases}E^{j-i} & j \geqslant i \\ \emptyset & j<i,\end{cases}
$$

with multiplication given by $*$, and identities $1 \in E^{0}$.

Proof. Define $\nabla^{n} \in \operatorname{Set}^{\Delta_{* *}^{\text {opp }}}$ by $\left(\nabla^{n}\right)_{r}:=\operatorname{Hom}_{\Delta_{* *}}(\mathbf{r}, \mathbf{n})$. If $U: \mathbb{S} \rightarrow \operatorname{Set}^{\Delta_{* *}^{\text {opp }}}$ is the forgetful functor, then define $b: U K \rightarrow K_{0} \times K_{0}$ by $x \mapsto\left(\left(\partial_{1}\right)^{n} x,\left(\partial_{0}\right)^{n} x\right)$, for $x \in K_{n}$. Then

$$
U \Delta^{n}=\coprod_{(i, j) \in \Delta_{0}^{n}} b^{-1}(i, j) \cong \coprod_{0 \leqslant i \leqslant j \leqslant n} \nabla^{j-i}
$$

For $\mathcal{C} \in \mathrm{Cat}_{n}, P_{n} \mathcal{C}$ decomposes in $\mathrm{Set}^{\Delta_{* *}}$ as

$$
P_{n} \mathcal{C}=\prod_{0 \leqslant i \leqslant j \leqslant n} \mathcal{C}(i, j)^{\nabla^{j-i}}
$$

where, for $K \in \operatorname{Set}^{\Delta_{* *}^{\text {opp }}}$ and a set $S$, we define $S^{K} \in \operatorname{Set}^{\Delta_{* *}}$ by $\left(S^{K}\right)_{r}=S^{K_{r}}$.

For $E \in Q M^{*}($ Set $)$ and $\mathcal{C} \in \mathrm{Cat}_{n}$, this implies that

$$
\begin{aligned}
\operatorname{Hom}_{\mathrm{Set}^{\Delta_{* *}}}\left(E, P_{n} \mathcal{C}\right) & =\prod_{0 \leqslant i \leqslant j \leqslant n} \operatorname{Hom}_{\mathrm{Set}^{\Delta_{* *}}}\left(E, \mathcal{C}(i, j)^{\nabla^{j-i}}\right) \\
& =\prod_{0 \leqslant i \leqslant j \leqslant n} \operatorname{Hom}_{\mathrm{Set}}\left(E^{j-i}, \mathcal{C}(i, j)\right) .
\end{aligned}
$$

Analysis of the product now gives the required result.

Definition 1.25. Given a category $\mathcal{C}$, a set $\mathcal{O}$ and a morphism $f: \mathcal{O} \rightarrow$ Ob $\mathcal{C}$, define $f^{-1} \mathcal{C}$ to be the category with objects $\mathcal{O}$ and morphisms $\left(f^{-1} \mathcal{C}\right)(a, b)=\mathcal{C}(f a, f b)$.

Lemma 1.26. For any category $\mathcal{C}$, there is a natural isomorphism

$$
(B \mathcal{C})_{n} \cong \coprod_{f \in(\mathrm{Ob} \mathcal{C})^{[0, n]}} \operatorname{MC}\left(P_{n} f^{-1} \mathcal{C}\right)
$$


Proof. Note that the right-hand side is just $\operatorname{Hom}_{\text {Cat }}\left(P_{n}^{*} \iota \bullet, \mathcal{C}\right)$. Since $(\iota \bullet)^{n}=\bullet$ for all $n$, we have $P_{n}^{*} \iota \bullet \cong \mathbf{n}$ by Lemma 1.24 , where we regard $\mathbf{n}$ as a category (with objects $[0, n]$, and a single morphism $i \rightarrow j$ whenever $i \leqslant j)$. This completes the proof, since the nerve is given by $(B \mathcal{C})_{n}=\operatorname{Hom}_{C a t}(\mathbf{n}, \mathcal{C})$.

We now generalise these results to more general categories.

Definition 1.27. Given $E \in \operatorname{Set}^{\Delta_{* *}}$ and $X \in \operatorname{Set}^{\Delta_{* *}^{\text {opp }}}$, define the set $X \times E$ to be the quotient of $\left\{(x, e) \in \coprod_{n} X_{n} \times E^{n}\right\}$ by the equivalence relation generated by

$$
\left(x, \partial^{i} e\right) \sim\left(\partial_{i} x, e\right),\left(x, \sigma^{i} e\right) \sim\left(\sigma_{i} x, e\right) .
$$

Definition 1.28. Given $K \in \mathbb{S}$ and $a, b \in K_{0}$, define $K(a, b) \in \operatorname{Set}^{\Delta_{* *}^{\text {opp }}}$ by setting

$$
K(a, b)_{n}:=\left\{x \in K_{n}:\left(\partial_{1}\right)^{n} x=a,\left(\partial_{0}\right)^{n} x=b\right\} .
$$

Lemma 1.29. Given a (small) category $\mathbb{I}$, the left adjoint $P_{B \mathbb{I}}^{*}$ to the functor

$$
P_{B \mathbb{I}}: \mathrm{Cat}_{\mathrm{ObI}} \rightarrow Q M^{*}(\mathrm{Set})
$$

is given by $\left(P_{B \mathbb{I}}^{*} E\right)(a, b)=(B \mathbb{I})(a, b) \times E$ for $a, b \in \mathrm{Ob} \mathbb{I}$, with the product given by $(x, e) \circ(y, f):=(x \star y, e * f)$, where $\star$ denotes concatenation of strings of morphisms in $\mathbb{I}$, and $*$ is the product on $E$.

Proof. For $K \in \mathbb{S}$, the category $P_{K}^{*} E$ has objects $K_{0}$. Morphisms are generated under composition by $K(a, b) \underset{\leftarrow}{E}$ in $\left(P_{K}^{*} E\right)(a, b)$ for $a, b \in K_{0}$, subject to the condition that for $e \in E^{m}, f \in E^{n}$ and $x \in K_{m+n}$,

$$
\left.(x, e * f) \sim\left(\left(\partial_{m+1}\right)^{n} x, e\right) \circ\left(\partial_{0}\right)^{m} x, f\right) .
$$

When $K=\mathbb{I}$, the map $\left(\left(\partial_{m+1}\right)^{n},\left(\partial_{0}\right)^{m}\right): K_{m+n} \rightarrow K_{m} \times\left(\partial_{0}\right)^{m}, K_{0},\left(\partial_{1}\right)^{n} K_{n}$ is an isomorphism, so any product of generators is a generator, giving the required result.

Lemma 1.30. For any category $\mathcal{C}$, there is a natural isomorphism

$$
\operatorname{Hom}_{\mathrm{Cat}}(\mathbb{I}, \mathcal{C}) \cong \coprod_{f \in(\mathrm{Ob \mathcal {C }})^{\mathrm{Ob} \mathbb{I}}} \operatorname{MC}\left(P_{B \mathbb{I}} f^{-1} \mathcal{C}\right)
$$

Proof. The proof of Lemma 1.26 carries over, noting that $P_{B \mathbb{I}}^{*} \iota \bumpeq \mathbb{I}$.

1.5.2. Quasi-descent data

Definition 1.31. Given $K \in \mathbb{S}$, define $P_{K}: Q \operatorname{Dat}_{K_{0}} \rightarrow Q M M^{*}($ Set $)$ by

$$
P_{K}(\mathcal{D})^{a, b}=\prod_{x \in K_{b}} \mathcal{D}\left(\left(\partial_{0}\right)^{b} x,\left(\partial_{1}\right)^{b} x\right)^{a},
$$

with operations

$$
\begin{aligned}
\partial_{v}^{i}(e)(x) & :=e\left(\partial_{i} x\right) \\
\sigma_{v}^{j}(e) & :=e\left(\sigma_{j} y\right) \\
(f * e)(z) & :=f\left(\left(\partial_{b+1}\right)^{b^{\prime}} z\right) * e\left(\left(\partial_{0}\right)^{b} z\right) .
\end{aligned}
$$

for $f \in P_{K}(\mathcal{C})^{a, b}, e \in P_{K}(\mathcal{C})^{a^{\prime}, b^{\prime}}$. The horizontal operations are $\partial_{h}^{i}=\partial_{\mathcal{D}}^{i}, \sigma_{h}^{i}=\sigma_{\mathcal{D}}^{i}$.

Note that $P_{n}=P_{\Delta^{n}}$. 
Let $Q$ Dat $_{n}$ be the category of quasi-descent data on the $n+1$ objects $[0, n]$.

Lemma 1.32. The functor $P_{n}:$ QDat $_{n} \rightarrow Q M M^{*}$ (Set) has a left adjoint $P_{n}^{*}$, given by

$$
\left(P_{n}^{*}\right)(i, j)^{a}= \begin{cases}E^{a, j-i} & j \geqslant i \\ \emptyset & j<i,\end{cases}
$$

with multiplication given by $*$, identities $1 \in E^{00}$, and operations $\partial_{h}^{i}, \sigma_{h}^{i}$.

Proof. The proof of Lemma 1.24 adapts to this generality.

Definition 1.33. Given a quasi-descent datum $\mathcal{D}$, a set $\mathcal{O}$ and a morphism $f: \mathcal{O} \rightarrow$ $\mathrm{Ob} \mathcal{D}$, define $f^{-1} \mathcal{D}$ to be the quasi-descent datum with objects $\mathcal{O}$ and morphisms $\left(f^{-1} \mathcal{D}\right)(a, b)^{i}=\mathcal{D}(f a, f b)^{i}$.

Lemma 1.34. For any quasi-descent datum $\mathcal{D}$, there is a natural isomorphism

$$
(B \operatorname{alg} \mathcal{D})_{n} \cong \coprod_{f \in(\mathrm{Ob} \mathcal{D})^{[0, n]}} \mathrm{MC}\left(\operatorname{diag} P_{n} f^{-1} \mathcal{D}\right)
$$

Proof. By Lemma 1.15, the left-hand side is $\operatorname{Hom}_{\text {Cat }}(\mathbf{n}, \operatorname{alg} \mathcal{D}) \cong \operatorname{Hom}_{Q D a t}\left(\operatorname{alg}^{*} \mathbf{n}, \mathcal{D}\right)$. Meanwhile, the right-hand side is $\operatorname{Hom}_{Q \text { Dat }}\left(P_{n}^{*} \operatorname{diag}^{*} \iota \bullet, \mathcal{D}\right)$, so it suffices to show that $\operatorname{alg}^{*} \mathbf{n} \cong P_{n}^{*} \operatorname{diag}^{*} \iota \bullet$. By Corollary $1.22, P_{n}^{*} \operatorname{diag}^{*} \iota \bullet=P_{n}^{*} \iota(\bullet, \bullet)$, and Lemma 1.32 shows that $\left(P_{n}^{*} \iota(\bullet, \bullet)\right)(i, j)^{a}$ is $\bullet$ for $j \geqslant i$ and $\emptyset$ for $j<i$, so $P_{n}^{*} \iota(\bullet, \bullet)=\operatorname{alg}^{*} \mathbf{n}$.

Lemma 1.35. Given a category $\mathbb{I}$, the left adjoint $P_{B \mathbb{I}}^{*}$ to the functor

$$
P_{B \mathbb{I}}: Q \text { Dat }_{\mathrm{Ob} \mathbb{I}} \rightarrow Q M M^{*}(\text { Set })
$$

is given by

$$
\left(P_{B \mathbb{I}}^{*} E\right)(a, b)^{n}=(B \mathbb{I})(a, b) \underset{\leftarrow}{\longleftarrow} E^{n}
$$

for $a, b \in \mathrm{Ob} \mathbb{I}$, with the product given by

$$
(x, e) \circ(y, f):=(x \star y, e * f),
$$

where $\star$ denotes concatenation of strings of morphisms in $\mathbb{I}$, and $*$ is the product on $E$.

Proof. The proof of Lemma 1.29 adapts to this generality.

Lemma 1.36. For any quasi-descent datum $\mathcal{D}$ and a category $\mathbb{I}$, there is a natural isomorphism

$$
\operatorname{Hom}_{\mathrm{Cat}}(\mathbb{I}, \operatorname{alg} \mathcal{D}) \cong \coprod_{f \in(\mathrm{Ob} \mathcal{D})^{\mathrm{Ob} \mathbb{I}}} \mathrm{MC}\left(\operatorname{diag} P_{B \mathbb{I}} f^{-1} \mathcal{D}\right)
$$

Proof. The proof of Lemma 1.34 carries over, noting that $P_{B \mathbb{I}}^{*} \iota(\bullet, \bullet) \cong \operatorname{alg*} \mathbb{I}$. 


\section{Algebras, coalgebras and bialgebras}

\subsection{Algebras and coalgebras}

Definition 2.1. A monad (or triple) on a category $\mathcal{B}$ is a monoid in the category of endofunctors of $\mathcal{B}$ (with the monoidal structure given by composition of functors). A comonad (or cotriple) is a comonoid in the category of endofunctors of $\mathcal{B}$.

Lemma 2.2. Take an adjunction

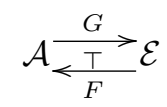

with unit $\eta:$ id $\rightarrow G F$ and co-unit $\varepsilon: F G \rightarrow$ id. Then $\top:=G F$ is a monad on $\mathcal{E}$ with unit $\eta$ and multiplication $\mu:=G \varepsilon F$, while $\perp:=F G$ is a comonad on $\mathcal{A}$, with co-unit $\varepsilon$ and comultiplication $\Delta:=F \eta G$.

Proof. For the monad $T$, this is [Mac, §VI.1], with the comonadic results following by duality.

Definition 2.3. Given a monad $(\top, \mu, \eta)$ on a category $\mathcal{E}$, define the category $\mathcal{E}^{\top}$ of T-algebras to have objects $\top E \stackrel{\theta}{\rightarrow} E$ (for $E \in \mathcal{E}$ ), such that $\theta \circ \eta_{E}=$ id and $\theta \circ \top \theta=$ $\theta \circ \mu_{E}$.

A morphism

$$
g:\left(\top E_{1} \stackrel{\theta}{\rightarrow} E_{1}\right) \rightarrow\left(\top E_{2} \stackrel{\phi}{\rightarrow} E_{2}\right)
$$

of $\top$-algebras is a morphism $g: E_{1} \rightarrow E_{2}$ in $\mathcal{E}$ such that $\phi \circ \top g=g \circ \theta$.

We define the comparison functor $K: \mathcal{A} \rightarrow \mathcal{E}^{\top}$ by

$$
B \mapsto\left(G F G B \stackrel{G \varepsilon_{B}}{\longrightarrow} G B\right)
$$

on objects, and $K(g)=G(g)$ on morphisms.

Definition 2.4. The adjunction

$$
\mathcal{A}_{\underset{F}{\stackrel{T}{\longrightarrow}} \mathcal{E}}^{\stackrel{G}{\longrightarrow}}
$$

is said to be monadic (or tripleable) if $K: \mathcal{A} \rightarrow \mathcal{E}^{\top}$ is an equivalence.

Examples 2.5. Intuitively, monadic adjunctions correspond to algebraic theories, such as the adjunction

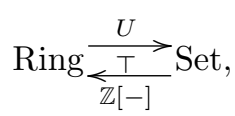

between rings and sets, $U$ being the forgetful functor. Other examples are $k$-algebras over $k$-vector spaces, or groups over sets.

Definition 2.6. Dually, given a comonad $(\perp, \Delta, \varepsilon)$ on a category $\mathcal{A}$, we define the category $\mathcal{A}_{\perp}$ of $\perp$-coalgebras by

$$
\left(\mathcal{A}_{\perp}\right)^{\mathrm{opp}}:=\left(\mathcal{A}^{\mathrm{opp}}\right)^{\perp},
$$

noting that $\perp$ is a monad on $\mathcal{A}^{\text {opp }}$. The adjunction of Lemma 2.2 is said to be comonadic (or cotripleable) if the adjunction on opposite categories is monadic. 
Examples 2.7. If $X$ is a topological space (or any site with enough points) and $X^{\prime}$ is the set of points of $X$, let $u: X^{\prime} \rightarrow X$ be the associated morphism. Then the adjunction

$$
\operatorname{Shf}\left(X^{\prime}\right) \underset{u^{-1}}{\stackrel{u_{*}}{\underset{\top}{\longrightarrow}}} \operatorname{Shf}(X),
$$

on the associated categories of sheaves is comonadic, so $\operatorname{Shf}(X)$ is equivalent to $u^{-1} u_{*^{-}}$ coalgebras in the category $\operatorname{Shf}\left(X^{\prime}\right)$ of sheaves (or equivalently presheaves) on $X^{\prime}$.

A more prosaic example is that for any $\operatorname{ring} A$, the category of $A$-coalgebras is comonadic over the category of $A$-modules.

\subsection{Quasi-descent data from monads}

Given a monad $(\top, \mu, \eta)$ on a category $\mathcal{B}$, and an object $B \in \mathcal{B}$, there is a quasicomonoid $E(B)$ given by

$$
E^{n}(B)=\operatorname{Hom}_{\mathcal{B}}\left(\top^{n} B, B\right)
$$

in (Set, $\times)$, with product $g * h=g \circ \top^{n} h$, and for $g \in E^{n}(B)$,

$$
\begin{aligned}
& \partial^{i}(g)=g \circ \top^{i-1} \mu_{\top n-i} B \\
& \sigma^{i}(g)=g \circ \top^{i} \eta_{\top n-i-1} B .
\end{aligned}
$$

If we replace $\mathcal{B}$ with a simplicial category, then $E(B)$ becomes a quasi-comonoid in $(\mathbb{S}, \times)$. Note that these constructions also all work for a comonad $(\perp, \Delta, \varepsilon)$, by contravariance.

Lemma 2.8. Given an object $B \in \mathcal{B}$, we see that the set of $\top$-algebra structures on $B$ is $\mathrm{MC}(E(B))$.

Proof. This follows immediately from the explicit description in Definition 1.7.

Proposition 2.9. Given a monad $(\top, \mu, \eta)$ (resp. a comonad $(\perp, \Delta, \varepsilon)$ ) on a category

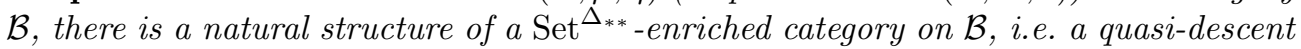
datum on objects $\mathrm{Ob} \mathcal{B}$.

Proof. Set

$$
\mathcal{H o m}\left(B, B^{\prime}\right)^{n}:=\operatorname{Hom}_{\mathcal{B}}\left(\top^{n} B, B^{\prime}\right) \quad\left(\operatorname{resp} . \operatorname{Hom}_{\mathcal{B}}\left(B, \perp^{n} B^{\prime}\right)\right),
$$

with product and operations as above.

Proposition 2.10. The category $\mathcal{B}^{\top}$ (resp. $\mathcal{B}_{\perp}$ ) of $\top$-algebras (resp. $\perp$-coalgebras) on $\mathcal{B}$ is isomorphic to the image under the functor alg: QDat $\rightarrow$ Cat (Definition 1.14) of the quasi-descent datum on $\mathcal{B}$ given in Proposition 2.9.

Proof. This follows immediately from the definitions.

\subsection{Bialgebras}

We now show how a bialgebraic structure on a category gives rise to a quasi-descent datum. 
As in [VO, $\S I V]$, take a category $\mathcal{B}$ equipped with both a monad $(\top, \mu, \eta)$ and a comonad $(\perp, \Delta, \varepsilon)$, together with a distributivity transformation $\lambda: \top \perp \Longrightarrow \perp \top$ for which the following diagrams commute:
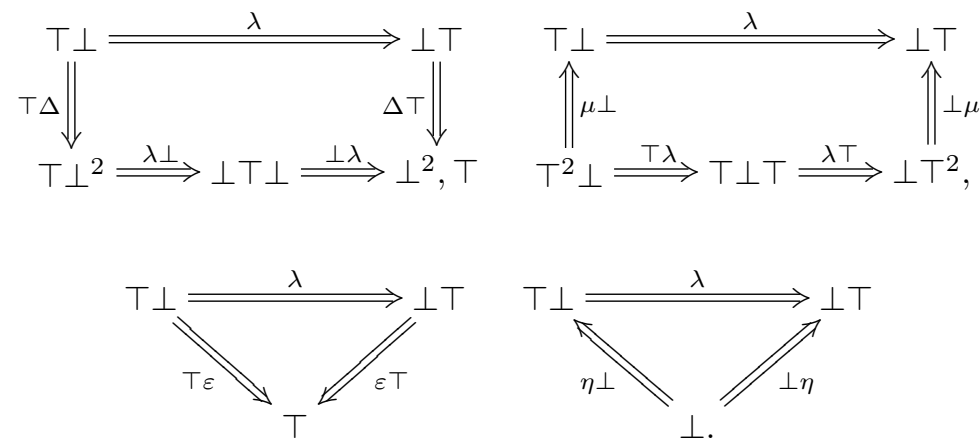

Definition 2.11. Given a distributive monad-comonad pair $(\top, \perp)$ on a category $\mathcal{B}$, define the category $\mathcal{B}_{\perp}^{\top}$ of bialgebras as follows. The objects of $\mathcal{B}_{\perp}^{\top}$ are triples $(\alpha, B, \beta)$ with $(\top B \stackrel{\alpha}{\rightarrow} B)$ an object of $\mathcal{B}^{\top}$ and $B \stackrel{\beta}{\rightarrow} \perp B$ an object of $\mathcal{B}_{\perp}$, such that the composition $(\beta \circ \alpha): \top B \rightarrow \perp B$ agrees with the composition

$$
\top B \stackrel{\top \beta}{\longrightarrow} \top \perp B \stackrel{\lambda}{\longrightarrow} \perp \top B \stackrel{\perp \alpha}{\longrightarrow} \perp B .
$$

A morphism $f:(\alpha, B, \beta) \rightarrow\left(\alpha^{\prime}, B^{\prime}, \beta^{\prime}\right)$ is a morphism $f: B \rightarrow B^{\prime}$ in $\mathcal{B}$ such that $\alpha^{\prime} \circ \top f=f \circ \alpha$ and $\beta^{\prime} \circ f=\perp f \circ \beta$.

Proposition 2.12. The data above give $\mathcal{B}$ the natural structure of a $\mathrm{Set}^{\Delta_{* *}}$-enriched category, with

$$
\mathcal{H o m}_{\mathcal{B}}\left(B, B^{\prime}\right)^{n}=\operatorname{Hom}_{\mathcal{B}}\left(\top^{n} B, \perp^{n} B^{\prime}\right)
$$

Proof. We follow [Pri2] in describing the operations. Since $\lambda$ is natural,

$$
(\lambda \perp \top) \circ(\top \perp \lambda)=(\perp \top \lambda) \circ(\lambda \top \perp) .
$$

Therefore any composition of $\lambda$ 's gives us the same canonical map

$$
\lambda_{m}^{n}: \top^{m} \perp^{n} \rightarrow \perp^{n} \top^{m},
$$

and we define the product on $\mathcal{H o m}\left(B^{\prime}, B^{\prime \prime}\right)^{m} \times \mathcal{H o m}\left(B, B^{\prime}\right)^{n} \rightarrow \mathcal{H} \operatorname{Hom}\left(B, B^{\prime \prime}\right)^{m+n}$ by

$$
g * h=\perp^{n}(g) \circ \lambda_{m}^{n} \circ \top^{m}(h) .
$$

The other operations are given by

$$
\begin{aligned}
& \partial^{i}(g)=\perp^{n-i} \Delta_{\perp^{i-1} B} \circ g \circ \top^{i-1} \mu_{\top_{\mathrm{h}}^{n-i} B}, \\
& \sigma^{i}(g)=\perp^{n-i-1} \epsilon_{\perp^{i} B} \circ g \circ \top^{i} \eta_{\top^{n-i-1} B} .
\end{aligned}
$$

To understand how the data $(\top, \perp, \eta, \mu, \varepsilon, \Delta, \lambda)$ above occur naturally, note that, 
by $[$ VO, $\S I V]$ or $[$ Pri2, $\S 2]$, these data are equivalent to a diagram

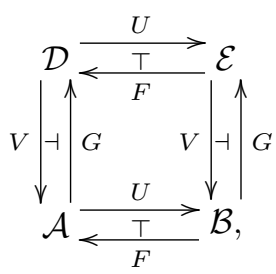

with $F \dashv U$ monadic, $G \vdash V$ comonadic and $U, V$ commuting with everything (although $G$ and $F$ need not commute). The associated monad on $\mathcal{B}$ is $\top=U F$, and the comonad $\perp=V G$. Distributivity ensures that $\mathcal{D} \simeq \mathcal{E}^{\top} \simeq\left(\mathcal{B}_{\perp}\right)^{\top}$ and $\mathcal{D} \simeq \mathcal{A}_{\perp} \simeq$ $\left(\mathcal{B}^{\top}\right)_{\perp}$. In other words, $\mathcal{D} \simeq \mathcal{B}_{\perp}^{\top}$. The functors $F$ are both free ${ }^{\top}$-algebra functors, while the functors $G$ are both cofree $\perp$-coalgebra functors.

Example 2.13. If $X$ is a topological space (or any site with enough points) and $X^{\prime}$ is the set of points of $X$, let $\mathcal{D}$ be the category of sheaves of rings on $X$. If $\mathcal{B}$ is the category of sheaves (or equivalently presheaves) of sets on $X^{\prime}$, then the description above characterises $\mathcal{D}$ as a category of bialgebras over $\mathcal{B}$, with the comonad being $u^{-1} u_{*}$ for $u: X^{\prime} \rightarrow X$ and the monad being the free polynomial functor.

Proposition 2.14. The category $\mathcal{B}_{\perp}^{\top}$ of $(\top, \perp)$-algebras on $\mathcal{B}$ is isomorphic to the image under the functor alg: QDat $\rightarrow$ Cat (Definition 1.14) of the quasi-descent datum $\tilde{\mathcal{B}}$ on $\mathcal{B}$ given in Proposition 2.12.

Proof. This is essentially [Pri2, Theorem 2.2]. Note that $\operatorname{alg}(\tilde{\mathcal{B}})$ arises naturally as

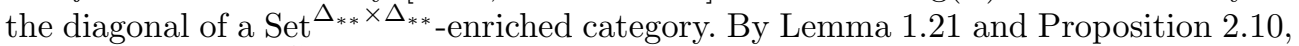
the objects of $\operatorname{alg}(\tilde{\mathcal{B}})$ over $B \in \mathcal{B}$ correspond to pairs $(\alpha, \beta)$, for $(\top B \stackrel{\alpha}{\longrightarrow} B) \in \mathcal{B}^{\top}$ and $(B \stackrel{\beta}{\rightarrow} \perp B) \in \mathcal{B}_{\perp}$ satisfying the conditions of Definition 2.11. The description of the morphisms follows similarly.

\section{Simplicial structures}

\subsection{Simplicial quasi-comonoids}

We now study the structure of the category of simplicial quasi-comonoids introduced in Definition 1.4.

Proposition 3.1. There is a cofibrantly generated Reedy simplicial model structure on $\mathbb{S}^{\Delta_{* *}}$ in which a map $f: X \rightarrow Y$ is

1. a weak equivalence if the maps $f^{n}: X^{n} \rightarrow Y^{n}$ are all weak equivalences,

2. a cofibration if the maps $f^{n}: X^{n} \rightarrow Y^{n}$ are all injective.

Proof. The category $\Delta_{* *}$ naturally has the structure of a Reedy category, with $\Delta_{* *,+}$ and $\Delta_{* *,-}$ the subcategories of injective and surjective maps, thus giving $\mathbb{S}^{\Delta_{* *}}$ a Reedy model structure. Since $\Delta_{* *,-}=\Delta_{-}$, the matching objects in $\mathcal{C}^{\Delta}$ and $\mathcal{C}^{\Delta_{* *}}$ are isomorphic, so it follows that this model structure on $\mathbb{S}^{\Delta_{* *}}$ is cofibrantly generated, from the corresponding result for $\mathbb{S}^{\Delta}$ (as in [GJ, §VII.4]). 
It only remains to describe the cofibrations. A morphism $f$ is a cofibration if the $\Delta_{* *}$-latching maps are cofibrations in $\mathbb{S}$. This is equivalent to saying that, for all $i$, the $\Delta_{* *}$ latching maps

$$
L^{n}\left(f_{i}\right): X_{i}^{n+1} \cup_{L^{n}\left(X_{i}\right)} L^{n}\left(Y_{i}\right) \rightarrow Y_{i}^{n+1}
$$

are injective.

Now, under the comparison $\Delta_{* *} \cong \Delta_{0}^{\text {opp }}, X_{i} \in$ Set $^{\Delta_{* *}}$ corresponds to an augmented simplicial set $\left(\breve{X}_{i}\right)$. Thus injectivity of the latching maps says that $\left(\breve{X}_{i}\right)_{\geqslant 0} \rightarrow\left(\breve{Y}_{i}\right)_{\geqslant 0}$ is a cofibration of simplicial sets and that $\left(\breve{X}_{i}\right)_{-1} \rightarrow\left(\breve{Y}_{i}\right)_{-1}$ is injective. Since cofibrations in $\mathbb{S}$ are precisely levelwise injective maps, this is equivalent to saying that the maps $f_{i}^{n}: X_{i}^{n} \rightarrow Y_{i}^{n}$ are all injective.

Lemma 3.2. There is a cofibrantly generated simplicial model structure on $Q M^{*}(\mathbb{S})$ for which a morphism $f$ is a fibration or weak equivalence whenever the underlying map in $\mathbb{S}^{\Delta_{* *}}$ is so.

Proof. Since the forgetful functor $Q M^{*}(\mathbb{S}) \rightarrow \mathbb{S}^{\Delta_{* *}}$ preserves filtered direct limits and has a left adjoint $F$, for any finite object $I \in \mathbb{S}^{\Delta_{* *}}$, the object $F I$ is finite in $Q M^{*}(\mathbb{S})$, so a fortiori it permits the small object argument. The model structure on $\mathbb{S}^{\Delta_{* *}}$ is cofibrantly generated by finite objects, so [Hir, Theorem 11.3.2] gives the required model structure on $Q M^{*}(\mathbb{S})$.

Remark 3.3. Observe that the category of comonoids in $(\mathbb{S}, \times)$ is just $\mathbb{S}$ itself, since the comultiplication $\Delta: X \rightarrow X \times X$ is necessarily given by the diagonal. Thus there is a functor $\iota: \mathbb{S} \rightarrow Q M^{*}(\mathbb{S})$, given by $\iota(X)^{m}=\overbrace{X \times X \times \cdots \times X}^{m}$, sending the comonoid $X$ to its associated quasi-comonoid.

The following follows immediately from Definition 1.7:

Lemma 3.4. If $E \in Q M^{*}(\mathbb{S})$, and $\bullet$ denotes the constant simplicial set on one element, then

$$
\operatorname{MC}\left(E_{0}\right) \cong \operatorname{Hom}_{Q M^{*}(\mathbb{S})}(\iota \bullet, E) .
$$

Definition 3.5. Define $\underline{\mathrm{MC}}: Q M^{*}(\mathbb{S}) \rightarrow \mathbb{S}$ by

$$
\underline{\mathrm{MC}}(E) \subset \prod_{n \geqslant 0}\left(E^{n+1}\right)^{I^{n}}
$$

(where $I=\Delta^{1} \in \mathbb{S}$ ), consisting of those $\underline{\omega}$ satisfying:

$$
\begin{aligned}
\omega_{m}\left(s_{1}, \ldots, s_{m}\right) * \omega_{n}\left(t_{1}, \ldots, t_{n}\right) & =\omega_{m+n+1}\left(s_{1}, \ldots, s_{m}, 0, t_{1}, \ldots, t_{n}\right), \\
\partial^{i} \omega_{n}\left(t_{1}, \ldots, t_{n}\right) & =\omega_{n+1}\left(t_{1}, \ldots, t_{i-1}, 1, t_{i}, \ldots, t_{n}\right), \\
\sigma^{i} \omega_{n}\left(t_{1}, \ldots, t_{n}\right) & =\omega_{n-1}\left(t_{1}, \ldots, t_{i-1}, \min \left\{t_{i}, t_{i+1}\right\}, t_{i+2}, \ldots, t_{n}\right), \\
\sigma^{0} \omega_{n}\left(t_{1}, \ldots, t_{n}\right) & =\omega_{n-1}\left(t_{2}, \ldots, t_{n}\right), \\
\sigma^{n} \omega_{n}\left(t_{1}, \ldots, t_{n}\right) & =\omega_{n-1}\left(t_{1}, \ldots, t_{n-1}\right), \\
\sigma^{0} \omega_{0} & =1 .
\end{aligned}
$$

We will refer to these as the higher Maurer-Cartan relations. 
Define $\mathrm{MC}: Q M^{*}(\mathbb{S}) \rightarrow$ Set by $\mathrm{MC}(E)=\underline{\mathrm{MC}}(E)_{0}$, noting that this agrees with Definition 1.7 when $E \in Q M^{*}$ (Set). Also note that we can recover $\underline{\mathrm{MC}}$ from $\mathrm{MC}$, since $\underline{\mathrm{MC}}(E)_{n}=\operatorname{MC}\left(E^{\Delta^{n}}\right)$.

Remark 3.6. Given a distributive monad-comonad pair $(\top, \perp)$ on a simplicial category $\mathcal{B}$, and an object $B$ of $\mathcal{B}$, Proposition 2.12 gives $\underline{\mathcal{H o m}}_{\mathcal{B}}(B, B) \in Q M^{*}(\mathbb{S})$, and we then regard $\underline{\mathrm{MC}}\left(\underline{\mathcal{H o m}}_{\mathcal{B}}(B, B)\right)$ as being the space of strong homotopy $(\top, \perp)$-bialgebras over $B$. If $\perp$ is trivial, then this is essentially the same as Lada's definition of the space of strong homotopy T-bialgebras from [CLM] (see Remark 3.13 for differences).

Definition 3.7. We now define matching objects for $E \in Q M^{*}(\mathbb{S})$ by $M^{0} E:=\bullet$, $M^{1} E:=E^{0}$, and for $n \geqslant 2$

$$
M^{n} E=\left\{\left(e_{0}, e_{1}, \ldots, e_{n-1}\right) \in\left(E^{n-1}\right)^{n} \mid \sigma^{i} e_{j}=\sigma^{j-1} e_{i} \forall i<j\right\} .
$$

These correspond to the matching objects $M^{n-1} E$ of [GJ, Lemma VII.4.9], but we have renumbered for consistency with the wider theory of Reedy categories.

The definition of the Reedy model structure on $\mathbb{S}^{\Delta_{* *}}$ implies the following.

Lemma 3.8. A morphism $f: E \rightarrow F$ in $Q M^{*}(\mathbb{S})$ is a fibration (resp. a trivial fibration) if and only if the relative matching maps

$$
E^{n} \rightarrow F^{n} \times_{M^{n} F} M^{n} E
$$

are fibrations (resp. trivial fibrations) in $\mathbb{S}$.

Lemma 3.9. For any trivial fibration $E \rightarrow F$ in $Q M^{*}(\mathbb{S})$, the map $\underline{\mathrm{MC}}(E) \rightarrow \underline{\mathrm{MC}}(F)$ is a trivial fibration.

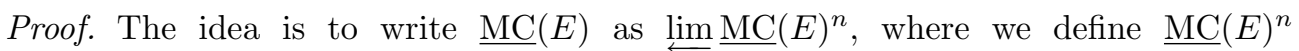
$\subset \prod_{0 \leqslant r \leqslant n}\left(E^{r+1}\right)^{I^{r}}$ satisfying the relations of Definition 3.5 above (to level $n$ ). We can summarise the Maurer-Cartan relations involving $\partial^{j}$ and $*$ as defining a function $f: \underline{\mathrm{MC}}(E)^{n-1} \rightarrow\left(E^{n+1}\right)^{\partial I^{n}}$, where $\partial I^{n}$ is the boundary of the simplicial set $I^{n}$. The relations involving $\sigma^{j}$ define a function $g: \underline{\mathrm{MC}}(E)^{n-1} \rightarrow\left(M^{n+1} E\right)^{I^{n}}$. If we set $\underline{\mathrm{MC}}(E)^{-1}=\bullet$, this allows us to write $\underline{\mathrm{MC}}(E)^{n}$ as the fibre product

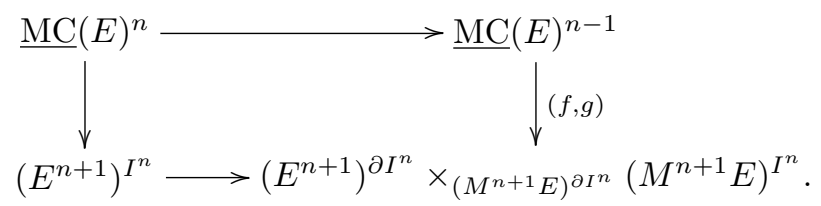

Since the pullback of a trivial fibration is a trivial fibration, it suffices to show that the map from $\left(E^{n+1}\right)^{I^{n}}$ to

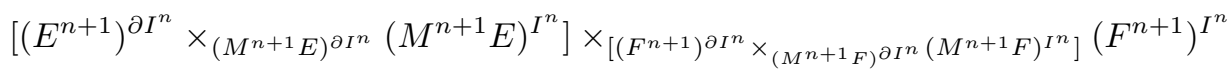

is a trivial fibration. By definition, the maps

$$
E^{n+1} \rightarrow M^{n+1} E \times_{M^{n+1} F} F^{n+1}
$$

are trivial fibrations. If $X \rightarrow Y$ is a trivial fibration, then $X^{I^{n}} \rightarrow X^{\partial I^{n}} \times_{Y^{\partial I^{n}}} Y^{I^{n}}$ is a trivial fibration, since $\partial I^{n} \rightarrow I^{n}$ is a cofibration in $\mathbb{S}$ (using the simplicial structure of $\mathbb{S})$. This gives the required result. 
Definition 3.10. Define an object $\Xi \in Q M^{*}(\mathbb{S})$ by $\Xi^{0}=\bullet$ and $\Xi^{n+1}=I^{n}$ for $n \geqslant 0$, where $I=\Delta^{1}$, with operations

$$
\begin{aligned}
\left(s_{1}, \ldots, s_{m}\right) *\left(t_{1}, \ldots, t_{n}\right) & =\left(s_{1}, \ldots, s_{m}, 0, t_{1}, \ldots, t_{n}\right) \\
\partial^{i}\left(t_{1}, \ldots, t_{n}\right) & =\left(t_{1}, \ldots, t_{i-1}, 1, t_{i}, \ldots, t_{n}\right), \\
\sigma^{i}\left(t_{1}, \ldots, t_{n}\right) & =\left(t_{1}, \ldots, t_{i-1}, \min \left\{t_{i}, t_{i+1}\right\}, t_{i+2}, \ldots, t_{n}\right), \\
\sigma^{0}\left(t_{1}, \ldots, t_{n}\right) & =\left(t_{2}, \ldots, t_{n}\right) \\
\sigma^{n}\left(t_{1}, \ldots, t_{n}\right) & =\left(t_{1}, \ldots, t_{n-1}\right) .
\end{aligned}
$$

Proposition 3.11. For $E \in Q M^{*}(\mathbb{S})$ fibrant, there is a natural weak equivalence

$$
\mathbf{R} \underline{\operatorname{Hom}}_{Q M^{*}(\mathbb{S})}(\iota \bullet, E) \simeq \underline{\mathrm{MC}}(E)
$$

in $\mathbb{S}$.

Proof. It follows from Definition 3.5 that $\underline{\mathrm{MC}}(E)=\underline{\operatorname{Hom}}_{Q M^{*}(\mathbb{S})}(\Xi, E)$.

Next, observe that the unique map $\Xi \rightarrow \iota \bullet$ is a weak equivalence, since the maps $I^{n} \rightarrow \bullet$ are weak equivalences. For any trivial fibration $E \rightarrow F$ in $Q M^{*}(\mathbb{S})$, Lemma 3.9 shows that

$$
\operatorname{Hom}_{Q M^{*}(\mathbb{S})}(\Xi, E) \rightarrow \operatorname{Hom}_{Q M^{*}(\mathbb{S})}(\Xi, F)
$$

is surjective, so $\Xi$ has the left lifting property with respect to trivial fibrations, making it a cofibrant replacement for $\iota \bullet$. Thus, for $E$ fibrant,

$$
\underline{\mathrm{MC}}(E) \simeq \mathbf{R}_{\operatorname{Hom}_{Q M}(\mathbb{S})}(\iota \bullet, E) .
$$

Corollary 3.12. The functor $\underline{\mathrm{MC}}: Q M^{*}(\mathbb{S}) \rightarrow \mathbb{S}$ is right Quillen.

Proof. Given a (trivial) fibration $E \rightarrow F$, the morphism $\underline{\mathrm{MC}}(E) \rightarrow \underline{\mathrm{MC}}(F)$ is

$$
\underline{\operatorname{Hom}}_{Q M^{*}(\mathbb{S})}(\Xi, E) \rightarrow \underline{\operatorname{Hom}}_{Q M^{*}(\mathbb{S})}(\Xi, F) .
$$

This is a (trivial) fibration, since $Q M^{*}(\mathbb{S})$ is a simplicial model category.

Remarks 3.13. Lada's definition of a strong homotopy algebra in $[\mathbf{C L M}]$ differs from Definition 3.5 in that it omits all of the degeneracy conditions except $\sigma^{0} \omega_{0}=0$. Proposition 3.11 would not be true if we omitted those degeneracy conditions.

Now, consider a $T$-algebra $A$ in topological spaces, and a retraction $r: A \rightarrow X$, with section $s$. Given a homotopy $h$ from $s r$ to $\mathrm{id}_{A}$, Lada constructs a system

$$
\left\{\omega_{n}: \top^{n+1} X \times|I|^{n} \rightarrow X\right\}
$$

by setting

$$
\omega_{n}\left(a_{1}, \ldots, a_{n}\right):=r \circ \varepsilon_{A} * h\left(a_{1}\right) * \varepsilon_{A} * h\left(a_{2}\right) * \cdots * h\left(a_{n}\right) * \varepsilon_{A} \circ s .
$$

If we impose the additional conditions that $r \circ h(a)=r, h(a) \circ s=s$ and $h(a)$ $\circ h(b)=h(\min (a, b))$, for all $a, b \in[0,1]$, then $\omega$ satisfies the higher Maurer-Cartan relations of Definition 3.5.

Moreover, a similar description holds for retracts $X$ of $(\top, \perp)$-bialgebras $A$, replacing $\varepsilon_{A}$ with $\eta_{A} \circ \varepsilon_{A}: \top A \rightarrow \perp A$.

Another way to look at this is that $|\Xi|$ is a cofibrant resolution of $\iota\left(\left|\Delta^{0}\right|\right)$ in $Q M^{*}$ (Top). An alternative (and possibly more natural) cofibrant replacement $\Phi$ of 
$\iota\left(\left|\Delta^{0}\right|\right)$ is given by $\Phi^{0}=\left|\Delta^{0}\right|$ and $\Phi^{n+1}=|I|^{n}$, with the same operations as $\Xi$, except that we replace the map min: $|I| \times|I| \rightarrow|I|$ with the map $(a, b) \mapsto a b$. Thus, for $E \in Q M^{*}(\mathrm{Top})$ and Sing: Top $\rightarrow \mathbb{S}$, the space $\mathrm{MC}(\operatorname{Sing} E)$ is equivalent to the subset of $\prod_{n \geqslant 0} \underline{\operatorname{Hom}}_{\text {Top }}\left(|I|^{n}, E^{n+1}\right)$ satisfying the conditions of Definition 3.5, except that we replace min with multiplication. The procedure above then allows us to construct a point $\omega$ of this space from a deformation retract, provided we modify the conditions above by requiring that the homotopy satisfies $h(a) \circ h(b)=h(a b)$.

Definition 3.14. For $E \in Q M^{*}(\mathbb{S})$ with $E^{0}$ a group (rather than just a monoid), there is an adjoint action of $E^{0}$ on $\underline{\mathrm{MC}}(E)$, given by $(g, \omega) \mapsto g^{-1} * \omega * g$. We then define $\operatorname{Del}(E)$ to be the homotopy quotient $\operatorname{Del}(E)=\left[\underline{\mathrm{MC}}(E) /{ }^{h} E^{0}\right]=\underline{\mathrm{MC}}(E) \times E^{0} W E^{0}$, for $W$ the universal cover of $B E^{0}=\bar{W} E^{0}$, as in [GJ, Ch. V.4].

\subsection{Simplicial categories}

Definition 3.15. Given a simplicial category $\mathcal{C}$, recall from [Ber1] that the category $\pi_{0} \mathcal{C}$ is defined to have the same objects as $\mathcal{C}$, with morphisms

$$
\operatorname{Hom}_{\pi_{0} \mathcal{C}}(x, y)=\pi_{0} \underline{\operatorname{Hom}}_{\mathcal{C}}(x, y) .
$$

A morphism in $\underline{\operatorname{Hom}}_{\mathcal{C}}(x, y)_{0}$ is said to be a homotopy equivalence if its image in $\pi_{0} \mathcal{C}$ is an isomorphism.

Lemma 3.16. There is a model structure on the category sCat of simplicial categories, in which a morphism $f: \mathcal{C} \rightarrow \mathcal{D}$ is

(W) a weak equivalence whenever

(W1) for any objects $a_{1}$ and $a_{2}$ in $\mathcal{C}$, the map $\underline{\operatorname{Hom}}_{\mathcal{C}}\left(a_{1}, a_{2}\right) \rightarrow \underline{\operatorname{Hom}}_{\mathcal{D}}\left(f a_{1}, f a_{2}\right)$ is a weak equivalence of simplicial sets;

(W2) the induced functor $\pi_{0} f: \pi_{0} \mathcal{C} \rightarrow \pi_{0} \mathcal{D}$ is an equivalence of categories.

$(F)$ a fibration whenever

(F1) for any objects $a_{1}$ and $a_{2}$ in $\mathcal{C}$, the map $\underline{\operatorname{Hom}}_{\mathcal{C}}\left(a_{1}, a_{2}\right) \rightarrow \underline{\operatorname{Hom}}_{\mathcal{D}}\left(f a_{1}, f a_{2}\right)$ is a fibration of simplicial sets;

(F2) for any objects $a_{1} \in \mathcal{C}, b \in \mathcal{D}$, and homotopy equivalence $e: f a_{1} \rightarrow b$ in $\mathcal{D}$, there is an object $a_{2} \in \mathcal{C}$ and a homotopy equivalence $d: a_{1} \rightarrow a_{2}$ in $\mathcal{C}$ such that $f d=e$.

Proof. See [Ber1, Theorem 1.1].

\subsection{Simplicial quasi-descent data}

Lemma 3.17. For a fixed set $\mathcal{O}$, there is a cofibrantly generated simplicial model category structure on $s Q D_{\text {Dat }} \mathcal{O}$ for which a morphism $f: \mathcal{D} \rightarrow \mathcal{D}^{\prime}$ is a fibration or a weak equivalence if and only if, for all $a, b \in \mathcal{O}$, the map

$$
f: \underline{\mathcal{H} o m}_{\mathcal{D}}(a, b) \rightarrow \underline{\mathcal{H o m}}_{\mathcal{D}^{\prime}}(a, b)
$$

is a Reedy fibration or a levelwise weak equivalence in $\mathbb{S}^{\Delta_{* *}}$.

Proof. Applying [Hir, Theorem 11.3.2] (as in the proof of Lemma 3.2) to the right adjoint functor $s Q$ Dat $_{\mathcal{O}} \rightarrow \mathbb{S}^{\Delta_{* *}}$ given by $\mathcal{D} \mapsto \prod_{a, b \in \mathcal{O}}{\underline{\mathcal{H} o m_{\mathcal{D}}}}_{\mathcal{D}}(a, b)$, we see that this is a model structure. 
The simplicial structure comes from the simplicial structure on $\mathbb{S}^{\Delta_{* *}}$, so

$$
\underline{\mathcal{H} o m}_{\mathcal{D}^{K}}(a, b):=\underline{\mathcal{H} o m}_{\mathcal{D}}(a, b)^{K} .
$$

We now consider the whole category $s Q D a t$, not just the subcategories on fixed objects.

Lemma 3.18. The functor $(-)^{0}: s Q$ Dat $\rightarrow s$ Cat is both a left and a right adjoint. The functor $\mathcal{D} \mapsto \prod_{a, b \in \mathrm{Ob} \mathcal{D}} \underline{\mathcal{H}}_{\mathcal{D}}(a, b)$ from $s Q D$ at to $\mathbb{S}^{\Delta_{* *}}$ is a right adjoint.

Proof. $(-)^{0}$ has left adjoint (id/ $\left.\emptyset\right)$ and right adjoint $\operatorname{alg}^{*}$, with the same formulae and reasoning as in Lemmas 1.13 and 1.17. The left adjoint to

$$
\mathcal{D} \mapsto \prod_{a, b \in \mathrm{Ob} \mathcal{D}} \underline{\mathcal{H}}_{\mathcal{E}}(a, b)
$$

is the functor $U: \mathbb{S}^{\Delta_{* *}} \rightarrow s Q$ Dat given by sending $X$ to the category with two objects $x, y$, and morphisms

$$
\underline{\mathcal{H o m}}(x, x)^{n}=\underline{\mathcal{H o m}}(y, y)^{n}:= \begin{cases}1 & n=0 \\ \emptyset & n>0,\end{cases}
$$

$\underline{\mathcal{H} o m}(x, y):=X$ and $\underline{\mathcal{H} o m}(y, x):=\emptyset$.

Proposition 3.19. There is a cofibrantly generated model structure on sQDat for which a morphism $f: \mathcal{D} \rightarrow \mathcal{E}$ is

(W) a weak equivalence if and only if

(W1) for all $a, b \in \mathrm{Ob} \mathcal{D}$, the map

$$
f: \underline{\mathcal{H o m}}_{\mathcal{D}}(a, b) \rightarrow \underline{\mathcal{H} o m}_{\mathcal{E}}(f a, f b)
$$

is a weak equivalence in $\mathbb{S}^{\Delta_{* *}}$, and

(W2) the morphism $\pi_{0}\left(f^{0}\right): \pi_{0}\left(\mathcal{D}^{0}\right) \rightarrow \pi_{0}\left(\mathcal{E}^{0}\right)$ is an equivalence of categories (for $\pi_{0} \mathcal{C}$ as in Definition 3.15);

(F) a fibration if and only if

(F1) for all $a, b \in \mathrm{Ob} \mathcal{D}$, the map

$$
f: \underline{\mathcal{H} o m}_{\mathcal{D}}(a, b) \rightarrow \underline{\mathcal{H} o m}_{\mathcal{E}}(f a, f b)
$$

is a Reedy fibration in $\mathbb{S}^{\Delta_{* *}}$, and

(F2) for any objects $a_{1} \in \mathcal{D}, b \in \mathcal{E}$ and a homotopy equivalence $e: f a_{1} \rightarrow b$ in $\mathcal{E}^{0}$, there exist an object $a_{2} \in \mathcal{E}$ and a homotopy equivalence $d: a_{1} \rightarrow a_{2}$ in $\mathcal{D}^{0}$ such that $f^{0} d=e$.

Proof. Note that these conditions are equivalent to saying that $f$ is a weak equivalence or fibration provided that both $f^{0}: \mathcal{D}^{0} \rightarrow \mathcal{E}^{0}$ and the maps $\underline{\mathcal{H}}_{\mathcal{D}}(a, b) \rightarrow$ $\underline{\mathcal{H o m}}_{\mathcal{E}}(f a, f b)$ are weak equivalences or fibrations. This follows because the functor $\mathbb{S}^{\Delta_{* *}} \rightarrow \mathbb{S}$ given by $X \mapsto X^{0}$ preserves both weak equivalences and fibrations (by definition of the Reedy model structure).

For $U$ as in the proof of Lemma 3.18, define $(I 1)$ to be the class consisting of the images under $U$ of the generating cofibrations from Proposition 3.1, and let (I2) be 
the single morphism $\emptyset \rightarrow \mathbf{0}$ from the category with no objects to the category with one object and no non-identity morphisms. Define $(I):=(I 1) \cup(I 2)$.

Define $(J 1)$ to be the class consisting of the images under $U$ of the generating trivial cofibrations from Proposition 3.1, and let $(J 2)$ be the image of the class $(A 2)$ from [Ber1] under the functor (id/ $\emptyset): s$ Cat $\rightarrow s Q$ Dat of Lemma 3.18. Define $(J):=(J 1) \cup(J 2)$.

For a class $C$ of morphisms, say that a morphism $f$ is $S$-injective if it has the left lifting property (LLP) with respect to S. From the adjoint property of the functor $U$, it follows that a morphism $f: \mathcal{D} \rightarrow \mathcal{E}$ is (J1)-injective, (resp. (I1)-injective) if and only if $f$ satisfies $(F 1)$ (resp. $(F 1)$ and $(W 1)$ ). The morphism $f$ is $(I 2)$-injective if and only if it is surjective on objects. By Lemma $3.18, f$ is $(J 2)$-injective if and only if $f^{0}: \mathcal{D}^{0} \rightarrow \mathcal{E}^{0}$ is $(A 2)$-injective.

Since cofibrations in $\mathbb{S}$, concentrated in degree 0 in $\mathbb{S}^{\Delta_{* *}}$, become cofibrations in the model structure of Proposition 3.1, the images under (id/ $\emptyset$ ) of the classes $(C 1)$, $(C 2),(A 1)$ and $(A 2)$ from [Ber1] lie in $(I 1),(I 2),(J 1)$ and $(J 2)$, respectively. It thus follows from [Ber1, Theorem 1.1] that for any $J$-injective (resp. $I$-injective) morphism $f: \mathcal{D} \rightarrow \mathcal{E}$, the morphism $f^{0}: \mathcal{D}^{0} \rightarrow \mathcal{E}^{0}$ is a fibration (resp. a trivial fibration) in $s$ Cat. Looking at the LLP in $\mathbb{S}^{\Delta_{* *}}$, we then deduce that $J$-injectives (resp. $I$-injectives) are precisely $(F)$ (resp. $(F) \cap(W))$ in $s Q$ Dat.

We now verify the conditions of [Hov, Theorem 2.1.19]. It is immediate that the class $(W)$ has the two-out-of-three property and is closed under retracts. The domains of $(I 2)$ and $(J 2)$ are small, and similarly to the proof of [Ber1, Theorem 1.1], the smallness of the generating (trivial) cofibrations in $\mathbb{S}^{\Delta_{* *}}$ means that the domains of $(I 1)$ and $(J 1)$ are small relative to $(I 1)$-cells and $(J 1)$-cells, respectively. Thus the domains of $(I)$ and $(J)$ are small relative to $(I)$-cells and $(J)$-cells, respectively. We have shown that the class of $(I)$-injectives is the intersection of $(W)$ with the class of $(J)$-injectives.

It remains only to show that all $(J)$-cells are in $(W)$ and are $(I)$-cofibrations. Since cofibrations in $\mathbb{S}$, concentrated in degree 0 in $\mathbb{S}^{\Delta_{* *}}$, become cofibrations in the model structure of Proposition 3.1, the functor (id/ $\emptyset$ ) maps cofibrations in $s$ Cat to $(I)$ cofibrations. Thus $(J 2)$-cells are $(I)$-cofibrations, since (id/ $\emptyset)$ preserves all colimits. Likewise, $U$ preserves all colimits and maps cofibrations to $(I)$-cofibrations, so $(J 1)$ cells are $(I)$-cofibrations, and therefore $(J)$-cells are $(I)$-cofibrations. Since $(J) \subset(W)$, it follows immediately from the definitions and the corresponding properties in $s$ Cat and $\mathbb{S}^{\Delta_{* *}}$ that all $(J)$-cells also lie in $(W)$.

Note that the functor $(-)^{0}: s Q D a t \rightarrow s$ Cat of Lemma 3.18 is then both left and right Quillen, while the functor $\mathcal{D} \mapsto \prod_{a, b \in \mathrm{Ob}} \mathcal{D} \underline{\mathcal{H o m}}_{\mathcal{D}}(a, b)$ from $s Q D a t \rightarrow \mathbb{S}^{\Delta_{* *}}$ is right Quillen.

Definition 3.20. We define model structures on $s Q D p d, s Q \operatorname{Dpd}_{\mathcal{O}}$ by requiring that a morphism $f$ is a weak equivalence or a fibration whenever the underlying map in $s Q$ Dat, $s Q$ Dat $_{\mathcal{O}}$ is so. We may apply [Hir, Theorem 11.3.2] to see that these are indeed model structures, since the forgetful functors preserve filtered colimits and have left adjoints (denoted by $\mathcal{D} \mapsto \mathcal{D}^{\text {Gpd }}$ ), given by formally inverting morphisms in $\mathcal{D}^{0}$. 


\subsection{Simplicial bicomonoids}

Lemma 3.21. There is a cofibrantly generated simplicial model structure on

$$
Q M M^{*}(\mathbb{S})
$$

for which a morphism $f$ is a fibration or weak equivalence whenever the underlying map in the Reedy model category $\mathbb{S}^{\Delta_{* *} \times \Delta_{* *}}$ is so.

Proof. The proof of Lemma 3.2 carries over.

Lemma 3.22. The diagonal functor $\operatorname{diag}: Q M M^{*}(\mathbb{S}) \rightarrow Q M^{*}(\mathbb{S})$ is right Quillen.

Proof. The Special Adjoint Functor Theorem ([Mac, Theorem V.8.1]) implies that diag: $Q M M^{*}(\mathbb{S}) \rightarrow Q M^{*}(\mathbb{S})$ has a left adjoint diag*. It therefore suffices to show that for any (trivial) fibration $f$ in $\mathbb{S}^{\Delta_{* *} \times \Delta_{* *}}$, the map $\operatorname{diag} f$ is a (trivial) fibration in $\mathbb{S}^{\Delta_{* *}}$.

Now, let $\Theta_{n}, \partial \Theta_{n} \in \operatorname{Set}^{\Delta_{* *}}$ be given by

$$
\operatorname{Hom}_{\operatorname{Set}^{\Delta_{* *}}}\left(\Theta_{n}, X\right)=X^{n} \text { and } \operatorname{Hom}_{\operatorname{Set}^{\Delta_{* *}}}\left(\partial \Theta_{n}, X\right)=M^{n} X \text {. }
$$

Similarly, let $\Theta_{i j}, \partial \Theta_{i j} \in \operatorname{Set}^{\Delta_{* *} \times \Delta_{* *}}$ be given by

$$
\operatorname{Hom}_{\operatorname{Set}^{\Delta_{* *} \times \Delta_{* *}}}\left(\Theta_{i j}, X\right)=X^{i, j} \text { and } \operatorname{Hom}_{\operatorname{Set}^{\Delta_{* *} \times \Delta_{* *}}}\left(\partial \Theta_{i j}, X\right)=M^{i, j} X \text {. }
$$

Latching object arguments (adapting [GJ] Proposition VII.1.7) show that the maps $\partial \Theta_{n} \subset \Theta_{n}$ generate all monomorphisms in Set $^{\Delta_{* *}}$, and likewise the maps $\partial \Theta_{i j} \subset \Theta_{i j}$ generate all monomorphisms in $\operatorname{Set}^{\Delta_{* *} \times \Delta_{* *}}$.

The diagonal functor diag: $\operatorname{Set}^{\Delta_{* *} \times \Delta_{* *}} \rightarrow$ Set $^{\Delta_{* *}}$ has a left adjoint diag*, and we just observe that this preserves monomorphisms (much like the case of bisimplicial sets considered in [GJ, Theorem IV.3.15]). Therefore the functor diag* $: \mathbb{S}^{\Delta_{* *}} \rightarrow \mathbb{S}^{\Delta_{* *} \times \Delta_{* *}}$ preserves Reedy (trivial) cofibrations, so is left Quillen, making diag right Quillen.

Note that we may regard $Q M^{*}(\mathbb{S})$ and $Q M M^{*}(\mathbb{S})$ as being simplicial diagrams in $Q M^{*}$ (Set) and $Q M M^{*}$ (Set), respectively. This will allow us to extend many of the constructions of Section 1 to the simplicial case.

\subsection{Nerves}

In Remark 3.6, we saw how $\underline{\mathrm{MC}}$ enables us to define the space of s.h. bialgebras over a fixed object. However, as was first noted in $[\mathbf{C L M}]$, there is no satisfactory general way to define morphisms of s.h. algebras. The bar construction of $[\mathbf{C L M}]$ gives a definition when the monad is an operad, but does not generalise to s.h. bialgebras. Instead, we will now introduce a space of s.h. I-diagrams of s.h. bialgebras for any small category $\mathbb{I}$, allowing us to mimic the nerve construction and thus to construct a simplicial space of s.h. bialgebras.

\subsection{1. $\mathcal{M C}$}

Definition 3.23. Given $K \in \mathbb{S}$, define the functor $P_{K}: s Q$ Dat $_{K_{0}} \rightarrow Q M M^{*}(\mathbb{S})$ by extending Definition 1.31 to simplicial sets. Let $P_{n}:=P_{\Delta^{n}}$; as in Lemma 1.32, $P_{n}$ has a left adjoint $P_{n}^{*}$.

Proposition 3.24. The functor $P_{K}: s Q$ Dat $_{K_{0}} \rightarrow Q M M^{*}(\mathbb{S})$ is right Quillen. 
Proof. Since $P_{K}$ is defined as a limit, it preserves arbitrary limits, so we just need to show that it preserves (trivial) fibrations. We may regard an object $X$ of $\mathbb{S}^{\Delta_{* *} \times \Delta_{* *}}$ as a $\Delta_{* *}$ diagram in $\mathbb{S}^{\Delta_{* *}}$, by $i \mapsto X^{i, \bullet}$. Denote the associated Reedy matching objects by $M_{\text {hor }}^{i} X \in \mathbb{S}^{\Delta_{* *}}$. Similarly there is a diagram $j \mapsto X^{\bullet, j}$, and we denote the associated matching objects by $M_{\text {ver }}^{j} X \in \mathbb{S}^{\Delta_{* *}}$. Note that the Reedy matching objects in $\mathbb{S}^{\Delta_{* *} \times \Delta_{* *}}$ are then given by

$$
M^{i j} X=\left(M_{\mathrm{hor}}^{i} X\right)^{j} \times_{M_{\mathrm{hor}}^{i}} M_{\mathrm{ver}}^{j} X\left(M_{\mathrm{ver}}^{j} X\right)^{i},
$$

as an immediate consequence of the characterisation of matching objects in [Hov, Definition 5.2.2]. by

Now, for $\mathcal{D} \in s Q$ Dat $_{K_{0}}$, the horizontal matching object $M_{\text {hor }}^{i} P_{K}(\mathcal{D})$ in $\mathbb{S}^{\Delta_{* *}}$ is given

$$
\left(M_{\mathrm{hor}}^{i} P_{K}(\mathcal{D})\right)^{j}=M^{i}\left(P_{K}(\mathcal{D})^{j}\right)=\prod_{x \in K_{j}} M^{i} \mathcal{D}\left(\left(\partial_{0}\right)^{j} x,\left(\partial_{1}\right)^{j} x\right) .
$$

Next, observe that for $X, K \in \mathbb{S}$, the object $S \in \mathbb{S}^{\Delta^{* *}}$ given by $S^{n}=X^{K_{n}}$ has matching object $M^{n} S$ given by

$$
M^{n} S=\left\{\left(f_{0}, f_{1}, \ldots, f_{n-1}\right) \in\left(X^{K_{n-1}}\right)^{n} \mid \sigma^{i} f_{j}=\sigma^{j-1} f_{i} \in X^{K_{n-2}} \forall i<j\right\} \cong X^{L_{n} K},
$$

where $L_{n} K$ is the $n$th simplicial latching object of $K$ (of [GJ, §VII.1]). Thus the vertical matching object $M_{\text {ver }}^{j} P_{K}(\mathcal{D})$ is given by

$$
\left(M_{\mathrm{ver}}^{j} P_{K}(\mathcal{D})\right)^{i}=\prod_{x \in L_{j} K} \mathcal{D}\left(\left(\partial_{0}\right)^{j} x,\left(\partial_{1}\right)^{j} x\right)^{i} .
$$

Therefore, since $L_{j} K \rightarrow K_{j}$ is always injective,

$$
M^{i j} P_{K}(\mathcal{D}) \cong\left(\prod_{x \in L_{j} K} \mathcal{D}\left(\left(\partial_{0}\right)^{j} x,\left(\partial_{1}\right)^{j} x\right)^{i}\right) \times\left(\prod_{x \in K_{j}-L_{j} K} M^{i} \mathcal{D}\left(\left(\partial_{0}\right)^{j} x,\left(\partial_{1}\right)^{j} x\right)\right),
$$

which yields the required result.

In fact, we may adapt this further to say that for any cofibration $i: J \hookrightarrow K$ in $\mathbb{S}$ and any (trivial) fibration $\mathcal{D} \rightarrow \mathcal{E}$ in $s Q$ Dat $_{K_{0}}$, the map

$$
P_{K}(\mathcal{D}) \rightarrow P_{K}(\mathcal{E}) \times_{P_{J}\left(i_{0}^{-1} \mathcal{E}\right)} P_{J}\left(i_{0}^{-1} \mathcal{D}\right)
$$

is a (trivial) fibration.

Definition 3.25. Define a functor $\mathcal{M C}: s Q$ Dat $\rightarrow s \mathbb{S}$ to bisimplicial sets by

$$
\mathcal{M C}(\mathcal{D})_{(n)}:=\coprod_{f:[0, n] \rightarrow \mathrm{Ob} \mathcal{D}} \underline{\mathrm{MC}}\left(\operatorname{diag} P_{n}\left(f^{-1} \mathcal{D}\right)\right) \in \mathbb{S} .
$$

Definition 3.26. Given a simplicial set $X$, we define $X \in s \mathbb{S}$ to be the constant space $X_{(n)}:=X$ for all $n$. By contrast, we define $X^{\text {hor }}$ by $X_{(n)}^{\text {hor }}:=X_{n}$.

Remark 3.27. Assume that $\mathcal{D}$ comes from a distributive monad-comonad pair $(\top, \perp)$ on a simplicial category $\mathcal{B}$, as in Proposition 2.12. Lemma 1.30 then allows us to think of $\mathcal{M C}(\mathcal{D})_{(n)}$ as being the space of s.h. n-diagrams of s.h. $(\top, \perp)$-bialgebras over $\mathcal{B}$, so $\mathcal{M C}$ is a kind of nerve construction. More generally, for any small category $\mathbb{I}$, we think of $\coprod_{f: \text { Ob } \mathbb{I} \rightarrow \mathrm{Ob} \mathcal{D}} \underline{\mathrm{MC}}\left(\operatorname{diag} P_{B \mathbb{I}}\left(f^{-1} \mathcal{D}\right)\right)$ as the space of s.h. $\mathbb{I}$-diagrams of s.h. $(\top, \perp)$-bialgebras over $\mathcal{B}$, noting that this is just $\underline{\operatorname{Hom}}_{s \mathbb{S}}\left((B \mathbb{I})^{\text {hor }}, \mathcal{M C}(\mathcal{D})\right)$. 
Lemma 3.28. Given a (trivial) fibration $f: \mathcal{D} \rightarrow \mathcal{E}$ in $s Q D a t$, the morphism

$$
\mathcal{M C}(\mathcal{D}) \rightarrow \mathcal{M C}(\mathcal{E}) \times{ }_{\operatorname{cosk}_{0}(\mathrm{Ob} \mathcal{E})^{\text {hor }} \operatorname{cosk}_{0}(\mathrm{Ob} \mathcal{D})^{\text {hor }}}
$$

is a (trivial) fibration in the Reedy category $s \mathbb{S}=\mathbb{S}^{\Delta^{\mathrm{opp}}}$, where $\operatorname{cosk}_{0}$ : Set $\rightarrow \mathbb{S}$ denotes the 0-coskeleton ([GJ, §IV.3]).

Proof. For any simplicial set $K, \operatorname{Hom}_{\mathbb{S}}\left(K, \operatorname{cosk}_{0} S\right)=S^{K_{0}} ;$ since $\left(\partial \Delta^{n}\right)_{0}=\left(\Delta^{n}\right)_{0}=$ $[0, n]$ (for $\partial \Delta^{n} \subset \Delta^{n}$ the boundary), the $n$th Reedy matching map of the morphism above is given by taking the coproduct over all $g:[0, n] \rightarrow \mathrm{Ob} \mathcal{D}$ of

$$
\begin{aligned}
& \underline{\mathrm{MC}}\left(\operatorname{diag} P_{n} g^{-1} \mathcal{D}\right) \rightarrow \\
& \quad \underline{\mathrm{MC}}\left(\operatorname{diag} P_{n} g^{-1}(\mathrm{Ob} f)^{-1} \mathcal{E}\right) \times_{\underline{\mathrm{MC}}\left(\operatorname{diag} P_{\partial \Delta^{n}} g^{-1}(\mathrm{Ob} f)^{-1} \mathcal{E}\right) \underline{\mathrm{MC}}\left(\operatorname{diag} P_{\partial \Delta^{n}} g^{-1} \mathcal{D}\right) .}
\end{aligned}
$$

Assume that $f$ is a (trivial) fibration. By the proof of Proposition 3.24,

$$
P_{n} g^{-1} \mathcal{D} \rightarrow\left(P_{n} g^{-1}(\mathrm{Ob} f)^{-1} \mathcal{E}\right) \times_{\left.P_{\partial \Delta^{n}} g^{-1}(\mathrm{Ob} f)^{-1} \mathcal{E}\right)}\left(P_{\partial \Delta^{n}} g^{-1} \mathcal{D}\right)
$$

is a (trivial) fibration in $Q M M^{*}(\mathbb{S})$, so Lemma 3.22 shows that

$$
\operatorname{diag} P_{n} g^{-1} \mathcal{D} \rightarrow\left(\operatorname{diag} P_{n} g^{-1}(\mathrm{Ob} f)^{-1} \mathcal{E}\right) \times_{\left.\operatorname{diag} P_{\partial \Delta^{n}} g^{-1}(\mathrm{Ob} f)^{-1} \mathcal{E}\right)}\left(\operatorname{diag} P_{\partial \Delta^{n}} g^{-1} \mathcal{D}\right)
$$

is a (trivial) fibration in $Q M^{*}(\mathbb{S})$, so Corollary 3.12 shows that the map above is a (trivial) fibration whenever $f$ is so.

Note that considering level 0 shows that $\mathcal{M C}: s Q D$ at $\rightarrow s \mathbb{S}$ is not right Quillen, since a fibration only maps to a fibration when it is surjective on objects, and a trivial fibration only maps to a trivial fibration when it is an isomorphism on objects.

\subsubsection{Del}

Definition 3.29. Let $s$ Gpd be the category of simplicial groupoids, i.e. the full subcategory of $(\mathrm{Gpd})^{\Delta^{\mathrm{opp}}}$ consisting of those $\Gamma$ for which the simplicial set $\mathrm{Ob} \Gamma$ of objects is constant. As in [GJ, $\S \mathrm{V} .7]$, this has a model structure in which a morphism is a weak equivalence or fibration whenever the corresponding morphism in $s$ Cat is so (for the model structure of Lemma 3.16), although the description simplifies considerably, since all morphisms in $\Gamma$ (and in particular homotopy equivalences) are isomorphisms.

Definition 3.30. Given $\Gamma \in s \mathrm{Gpd}$, define $\mathbb{S}(\Gamma)$ to be the category of simplicial $\Gamma$ representations. An object $X \in \mathbb{S}(\Gamma)$ consists of $X(a) \in \mathbb{S}$ for all objects $a$ of $\Gamma$ and distributive morphisms $\Gamma(a, b) \times X(b) \rightarrow X(a)$ in $\mathbb{S}$.

Lemma 3.31. For $\Gamma \in s Q D p d$, there is a natural $\Gamma^{0}$-representation in $\mathbb{S}$, given by mapping $a \in \mathrm{Ob} \Gamma$ to $\underline{\mathrm{MC}}(\Gamma(a, a))$. Denote this representation by $\underline{\mathrm{MC}}(\Gamma) \in \mathbb{S}\left(\Gamma^{0}\right)$.

Proof. We just need to define an associative action $\underline{\mathrm{MC}}(a) \times \Gamma^{0}(a, b) \rightarrow \underline{\mathrm{MC}}(b)$. As in Definition 3.14, the adjoint action $(\omega, g) \mapsto g^{-1} * \omega * g$ suffices.

Definition 3.32. Given a simplicial groupoid $\Gamma$, define the $\Gamma$-representation $W \Gamma$ by

$$
(W \Gamma)_{n}(a):=\coprod_{x_{0}, x_{1}, \ldots, x_{n}} \Gamma\left(a, x_{n}\right)_{n} \times \Gamma\left(x_{n}, x_{n-1}\right)_{n-1} \times \cdots \times \Gamma\left(x_{1}, x_{0}\right)_{0},
$$

for $a \in \mathrm{Ob} \Gamma$. As in [GJ, §V.4] (which considered only simplicial groups), this has 
operations:

$$
\begin{aligned}
& \partial_{i}\left(v_{n}, v_{n-1}, \ldots, v_{0}\right)= \begin{cases}\left(\partial_{i} v_{n}, \partial_{i-1} v_{n-1}, \ldots,\left(\partial_{0} v_{n-i}\right) v_{n-i-1}, v_{n-i-2}, \ldots, v_{0}\right) & i<n, \\
\left(\partial_{n} v_{n}, \partial_{n-1} v_{n-1}, \ldots, \partial_{1} v_{1}\right) & i=n,\end{cases} \\
& \sigma_{i}\left(v_{n}, v_{n-1}, \ldots, v_{0}\right)=\left(\sigma_{i} v_{n}, \sigma_{i-1} v_{n-1}, \ldots, \sigma_{0} v_{n-i}, 1, v_{n-i-1}, \ldots, v_{0}\right) .
\end{aligned}
$$

For $h \in \Gamma(a, b)$, the action is given by

$$
h\left(v_{n}, v_{n-1}, \ldots, v_{0}\right)=\left(h v_{n}, v_{n-1}, \ldots, v_{0}\right) .
$$

Definition 3.33. Given $\Gamma \in s \mathrm{Gpd}$, define

$$
\underset{\Gamma}{\operatorname{holim}}: \mathbb{S}(\Gamma) \rightarrow(\mathbb{S} \downarrow \bar{W} \Gamma)
$$

by $X \mapsto X \times^{\Gamma} W \Gamma$, where $\bar{W} \Gamma:=\bullet \times^{\Gamma} W \Gamma$ is a model for the classifying space of $\Gamma$ $([\mathbf{G J}, \S \mathrm{V} .7])$.

Lemma 3.34. The functor holim $: \mathbb{S}(\Gamma) \rightarrow(\mathbb{S} \downarrow \bar{W} \Gamma)$ is right Quillen, where fibrations and weak equivalences in $\mathbb{S}(\Gamma)$ are defined objectwise.

Proof. The proof of [GJ, Lemma VI.4.6], which takes the case when $\Gamma$ is a discrete groupoid, carries over to this generality. The left adjoint is given by

$$
X \mapsto X \times_{\bar{W} \Gamma} W \Gamma .
$$

Definition 3.35. Define a functor Del: $s Q D p d \rightarrow \mathbb{S}$ by

$$
\operatorname{Del}(\Gamma):=\underset{\Gamma^{0}}{\operatorname{holim}} \underset{\operatorname{MC}}{\operatorname{Mi}}(\Gamma),
$$

making use of the forgetful functor $\mathbb{S} \downarrow \bar{W} \Gamma \rightarrow \mathbb{S}$. Note that if $\Gamma$ has one object, then it may be regarded as an object of $Q M^{*}(\mathbb{S})$, and this definition is consistent with Definition 3.14 in this case.

Proposition 3.36. The functor $\operatorname{Del}: s Q D p d \rightarrow \mathbb{S}$ is right Quillen.

Proof. Since Del clearly preserves limits, we need only show that it preserves (trivial) fibrations. Given a (trivial) fibration $f: \mathcal{D} \rightarrow \mathcal{E}$ in $s Q D p d$, set $\mathcal{F}:=\mathcal{E} \times$ alg $^{*} \mathcal{E}^{0} \operatorname{alg}^{*} \mathcal{D}^{0}$, and observe that $f$ factors as the composition of the (trivial) fibrations $g: \mathcal{D} \rightarrow \mathcal{F}$, $h: \mathcal{F} \rightarrow \mathcal{E}$.

Now, $\underline{\operatorname{MC}}(\mathcal{D}) \rightarrow \underline{\operatorname{MC}}(\mathcal{F})$ is a morphism in $\mathbb{S}\left(\mathcal{D}^{0}\right)$ which is a (trivial) fibration by Corollary 3.12. Lemma 3.34 then implies that $\operatorname{Del}(\mathcal{D}) \rightarrow \operatorname{Del}(\mathcal{F})$ is a (trivial) fibration in $\mathbb{S}$, since $\operatorname{Ob}(\mathcal{D})=\operatorname{Ob}(\mathcal{F})$. The morphism $\operatorname{Del}(\mathcal{F}) \rightarrow \operatorname{Del}(\mathcal{E})$ is a pullback of $\operatorname{Del}\left(\operatorname{alg}^{*} \mathcal{D}^{0}\right) \rightarrow \operatorname{Del}\left(\operatorname{alg}^{*} \mathcal{E}^{0}\right)$, so it remains only to show that the latter is a (trivial) fibration.

Given $E \in Q M^{*}(\mathbb{S})$ with $E^{n}=E^{0}$ for all $n$, studying the degeneracy operations shows that $\underline{\mathrm{MC}}(E)=\{1\}$. Therefore $\underline{\mathrm{MC}}\left(\operatorname{alg}^{*} \mathcal{D}^{0}\right)$ is the constant $\mathcal{D}^{0}$-representation on the one-point set $\bullet$, so

$$
\operatorname{Del}\left(\operatorname{alg}^{*} \mathcal{D}^{0}\right)=\bullet \times^{\mathcal{D}^{0}} W \mathcal{D}^{0}=\bar{W} \mathcal{D}^{0}
$$

and similarly for $\operatorname{Del}\left(\operatorname{alg}^{*} \mathcal{E}^{0}\right)=\bar{W} \mathcal{E}^{0}$. The morphism $\mathcal{D}^{0} \rightarrow \mathcal{E}^{0}$ is a (trivial) fibration in $s \mathrm{Gpd}$, so $\bar{W} \mathcal{D}^{0} \rightarrow \bar{W} \mathcal{E}^{0}$ is a (trivial) fibration in $\mathbb{S}$, by [GJ, Theorem V.7.8]. 
Definition 3.37. Given $K \in \mathbb{S}$ and $\mathcal{D} \in s Q$ Dat, define $h(K, \mathcal{D}) \in s Q$ Dat by

$$
\operatorname{Ob}(h(K, \mathcal{D}))=\operatorname{Hom}_{\text {Set }}\left(K_{0}, \mathrm{Ob} \mathcal{D}\right)
$$

and

$$
h(K, \mathcal{D})(a, b)^{n}:=\prod_{x \in K_{n}} \mathcal{C}\left(a\left(\left(\partial_{0}\right)^{n} x\right) b\left(\left(\partial_{1}\right)^{n} x\right)\right),
$$

and note that $\mathcal{M C}(\mathcal{D})_{n}=\underline{\operatorname{MC}}\left(h\left(\Delta^{n}, \mathcal{D}\right)\right)$.

Definition 3.38. Define $\mathcal{D} \mathcal{E} \mathcal{L}: s Q \operatorname{Dpd} \rightarrow s \mathbb{S}$ by $\mathcal{D} \mathcal{E} \mathcal{L}(\Gamma)_{(n)}:=\operatorname{Del}\left(h\left(\Delta^{n}, \Gamma\right)\right)$.

Corollary 3.39. The functor $\mathcal{D E} \mathcal{L}: s Q \mathrm{Dpd} \rightarrow s \mathbb{S}$ is right Quillen.

Proof. This just combines Proposition 3.36 with the observation that for any (trivial) fibration $f: \mathcal{D} \rightarrow \mathcal{E}$ in $s Q D p d$, the morphism

$$
h\left(\Delta^{n}, \mathcal{D}\right) \rightarrow h\left(\Delta^{n}, \mathcal{E}\right) \times_{h\left(\partial \Delta^{n}, \mathcal{E}\right)} h\left(\partial \Delta^{n}, \mathcal{D}\right)
$$

is a (trivial) fibration in $s Q D$ at for all $n \geqslant 0$, which follows by combining Lemma 3.22 with the proof of Proposition 3.24.

\section{Abelian groups and cohomology}

In this section, we will investigate quasi-comonoids in abelian groups and in groupoids. The main motivation for this is that we can detect whether a simplicial set $X$ is contractible just by looking at $\pi_{f} X$ and $\mathrm{H}^{*}(X, \mathbb{Z})$, and we will now develop the corresponding notions for $Q M^{*}(\mathbb{S})$.

\subsection{Cosimplicial abelian groups}

Lemma 4.1. There is an equivalence between the category $Q M^{*}(\mathrm{Ab}, \times)$ of quasicomonoids in $(\mathrm{Ab}, \times)$ and the category $\mathrm{Ab}$ of cosimplicial complexes of abelian groups.

Proof. Take $A \in Q M^{*}(\mathrm{Ab}, \times)$. The operations $\partial^{i}$ and $\sigma^{i}$ on $A$ are necessarily $\mathbb{Z}$-linear. We enhance this to a cosimplicial structure by setting $\partial^{0} a=0_{1} * a, \partial^{m+1} a=a * 0_{1}$, for $a \in A^{m}$ and $0_{1}$ the group identity in $A^{1}$. To see that this satisfies the cosimplicial axioms, note that the properties of $*$ give that

$$
\begin{aligned}
\partial^{i+1} \partial^{0} a & =\partial^{0} \partial^{i} a & & \forall 1 \leqslant i \leqslant m, \\
\partial^{i} \partial^{m+1} a & =\partial^{m+2} \partial^{i} a & & \forall 1 \leqslant i \leqslant m, \\
\sigma^{i+1} \partial^{0} a & =\partial^{0} \sigma^{i} a & & \forall 0 \leqslant i<m, \\
\sigma^{i} \partial^{m+1} a & =\partial^{m} \sigma^{i} a & & \forall 0 \leqslant i<m .
\end{aligned}
$$

We also have $\partial^{0} \partial^{m+1} a=0_{1} * a * 0_{1}=\partial^{m+2} \partial^{0} a$, so it only remains to show that $\sigma^{0} \partial^{0}=$ id and $\sigma^{m} \partial^{m+1}=$ id and that $\partial^{0} \partial^{0}=\partial^{1} \partial^{0}$ and $\partial^{m+1} \partial^{m+1}=\partial^{m+2} \partial^{m+1}$. The first two conditions follow because $\sigma^{0} 0_{1}=0_{0}$, which is the identity for $*$, and the second two follow because $0_{1} * 0_{1}=0_{2}=\partial^{1} 0_{1}$. 
Since $A^{m} \times A^{n} \stackrel{*}{\rightarrow} A^{m+n}$ is linear, for $a, a^{\prime} \in A^{m}, b, b^{\prime} \in A^{n}$ we then have

$$
a * b^{\prime}+a^{\prime} * b=\left(a+a^{\prime}\right) *\left(b^{\prime}+b\right),
$$

so setting $a^{\prime}=0_{m}, b^{\prime}=0_{n}$ gives $a * b=a * 0_{n}+0_{m} * b$, and $0_{n}=0_{1}^{* n}$, so the product is necessarily the Alexander-Whitney cup product $a * b=\left(\partial^{m+1}\right)^{n} a+\left(\partial^{0}\right)^{m} b$, which is uniquely determined by the cosimplicial structure.

Definition 4.2. Let $s \mathrm{Ab}$ be the category of simplicial abelian groups and let $c s \mathrm{Ab}$ be the category of cosimplicial simplicial abelian groups.

Definition 4.3. Denote the left adjoint to the inclusion functor $c s A b \rightarrow Q M^{*}(\mathbb{S})$ by cot. This is left Quillen, and we denote the associated left-derived functor on homotopy categories by $\mathbf{L}$ cot.

Lemma 4.4. There is a cofibrantly generated simplicial model structure

$$
Q M^{*}(s \mathrm{Ab}, \times)
$$

in which a morphism is a fibration or a weak equivalence whenever the underlying map in $Q M^{*}(\mathbb{S})$ is so.

Proof. We may apply [Hir, Theorem 11.3.2] to the forgetful functor $Q M^{*}(s \mathrm{Ab}, \times) \rightarrow$ $Q M^{*}(\mathbb{S})$. This functor satisfies the Special Adjoint Functor Theorem ([Mac, Theorem V.8.2]), so it has a left adjoint (analogous to the free module generated by a set). It also preserves filtered direct limits, so admits the small object argument where necessary.

Lemma 4.5. There is an equivalence

$$
Q M^{*}(s \mathrm{Ab}, \times) \simeq c s \mathrm{Ab}
$$

of model categories, where csAb is given the Reedy model structure for cosimplicial objects in sAb (with its standard model structure).

Proof. Lemma 4.1 gives the equivalence of categories, by passing to simplicial diagrams. Now, $f$ is a weak equivalence in $Q M^{*}(s \mathrm{Ab}, \times)$ whenever each $f^{n}$ is a weak equivalence, and a fibration whenever $f$ is a Reedy fibration in $\mathbb{S}^{\Delta_{* *}}$. Since the matching objects for $\Delta$ and $\Delta_{* *}$ are the same, this means that the model structure of Lemma 4.4 is just the Reedy model structure on $(s \mathrm{Ab})^{\Delta}$, as they have the same weak equivalences and fibrations.

Definition 4.6. Let $N^{s}$ denote simplicial normalisation from simplicial abelian groups to non-negatively graded chain complexes, given by

$$
N^{s}(V)_{n}:=\bigcap_{i>0} \operatorname{ker}\left(\partial_{i}: V_{n} \rightarrow V_{n-1}\right),
$$

with differential $\partial_{0}$. Let $N_{c}$ denote cosimplicial conormalisation from cosimplicial abelian groups to non-negatively graded cochain complexes, given by

$$
N_{c}(V)^{n}:=\bigcap_{i \geqslant 0} \operatorname{ker}\left(\sigma^{i}: V^{n} \rightarrow V^{n-1}\right),
$$

with differential $\sum_{i}(-1)^{i} \partial^{i}$. By the Dold-Kan correspondence ([Wei, Theorem 8.4.1], passing to opposite categories and using [Wei, Lemma 8.3.7] in the cosimplicial case), 
these functors are both equivalences; let $D_{c}$ be the cosimplicial denormalisation functor, inverse to $N_{c}$.

Definition 4.7. Set $I=\Delta^{1}$, and for $n \geqslant 0$, let

$$
\Xi^{n}:=\left(\{1\} \times I^{n-1}\right) \cup \bigcup_{j>0}\left(I^{j} \times\{0,1\} \times I^{n-1-j}\right) \subset I^{n} \in \mathbb{S} ;
$$

for $n \geqslant 2$ this is given by removing the interior of $0 \times I^{n-1}$ from the boundary $\partial I^{n}$, while $\beth^{1}=\{1\}$ and $\beth^{0}=\emptyset$.

Let

$$
\mathbb{Z}\left(I^{n} / \beth^{n}\right):=\mathbb{Z}\left(I^{n}\right) / \mathbb{Z}\left(\beth^{n}\right) \in s \mathrm{Ab},
$$

where $\mathbb{Z}(S)$ is the free $\mathbb{Z}$-module generated by the set $S$, and let $\delta$ be the canonical map $\mathbb{Z}\left(I^{n-1} / \beth^{n-1}\right) \rightarrow \mathbb{Z}\left(I^{n} / \beth^{n}\right)$ arising from the map $I^{n-1} \rightarrow I^{n}$ given by $x \mapsto(0, x)$.

For any simplicial abelian group $W$, write

$$
W^{I^{n} / \beth^{n}}:=\operatorname{ker}\left(W^{I^{n}} \rightarrow W^{\beth^{n}}\right)=\underline{\operatorname{Hom}}_{s \mathrm{Ab}}\left(\mathbb{Z}\left(I^{n} / \beth^{n}\right), W\right)
$$

and let $\delta$ be the canonical map $W^{I^{n} / \beth^{n}} \rightarrow W^{I^{n-1} / \beth^{n-1}}$ dual to the map $\delta$ above.

Proposition 4.8. Given an abelian group object $E$ in $Q M^{*}(\mathbb{S})$, corresponding under Lemma 4.1 to the cosimplicial simplicial abelian group $\mathrm{C}(E)$, there is an isomorphism

$$
\underline{\mathrm{MC}}(E) \cong\left\{\eta \in \prod_{n=0}^{\infty} N_{c}^{n+1} \mathrm{C}(E)^{I^{n} / \beth^{n}}: d_{c} \eta_{n-1}=\delta \eta_{n}\right\} .
$$

Proof. As in Lemma 3.9, write $\underline{\mathrm{MC}}(E)=\varliminf_{n} \underline{\mathrm{MC}}(E)^{n}$, and assume that we are given an element

$$
\left(\omega_{0}, \ldots, \omega_{n-1}\right) \in \underline{\mathrm{MC}}(E)^{n-1} .
$$

The proof of Lemma 3.9 then gives rise to the data

$$
\beta_{n-1} \in M^{n+1} \mathrm{C}(E)^{I^{n}}, \quad \alpha_{n-1} \in \mathrm{C}^{n+1}(E)^{\partial I^{n}}
$$

(in the notation of Definition 3.7). By Lemma 3.9, the fibre of $\underline{\mathrm{MC}}(E)^{n} \rightarrow \underline{\mathrm{MC}}(E)^{n-1}$ over $\left(\omega_{0}, \ldots, \omega_{n-1}\right)$ is given by $\omega_{n} \in \mathrm{C}^{n+1}(E)^{I^{n}}$ compatibly lifting $\alpha_{n-1}, \overline{\beta_{n-1}}$ in the following diagram:

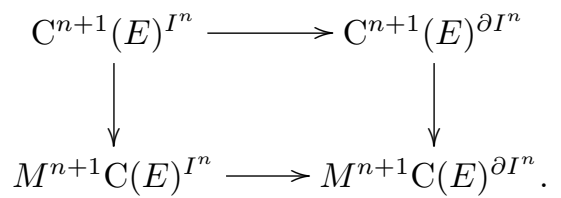

For any abelian cosimplicial abelian group $C^{\bullet}$, dualising [Wei, Lemma 8.3.7] gives a decomposition of the associated cochain complex as $C^{n}=N_{c}^{n}(C) \oplus\left(M^{\prime}\right)^{n}(C)$, where $N_{c}^{n}(C)=\cap_{i=0}^{n-1} \operatorname{ker} \sigma^{i}$, and $\left(M^{\prime}\right)^{n}(C)=\sum_{i=1}^{n} \partial^{i} C^{n-1}$, so the commutative diagram 
becomes

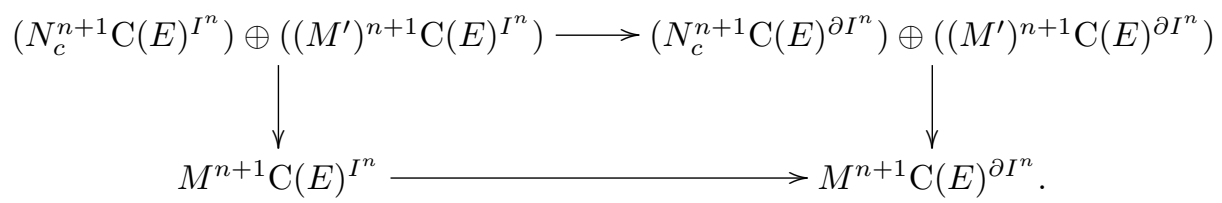

Moreover, $\underline{\sigma}:\left(M^{\prime}\right)^{n} C \rightarrow M^{n} C$ is an isomorphism, and we will denote the inverse by $a \mapsto \tilde{a}$. Thus the problem of constructing $\omega_{n}$ reduces to seeking an element $\eta_{n}=\omega_{n}-$ $\tilde{\beta}_{n-1} \in N_{c}^{n+1} \mathrm{C}(E)^{I^{n}}$ lifting $\operatorname{pr}_{N}\left(\alpha_{n-1}\right) \in N_{c}^{n+1} \mathrm{C}(E)^{\partial I^{n}}$, where

$$
\operatorname{pr}_{N}: C \rightarrow N_{c}(C)
$$

is the projection given by annihilating $M^{\prime}(C)$.

Now, $\alpha_{n-1}$ is defined by

$$
\begin{aligned}
\alpha_{n-1}\left(t_{1}, \ldots, t_{i-1}, 0, t_{i+1}, \ldots, t_{n}\right)= & \left(\partial^{i+1}\right)^{n-i+1} \omega_{i-1}\left(t_{1}, \ldots, t_{i-1}\right) \\
& +\left(\partial^{0}\right)^{i} \omega_{n-i}\left(t_{i+1}, \ldots, t_{n}\right) \\
\alpha_{n-1}\left(t_{1}, \ldots, t_{i-1}, 1, t_{i+1}, \ldots, t_{n}\right)= & \partial^{i} \omega_{n-1}\left(t_{1}, \ldots, t_{i-1}, t_{i+1}, \ldots, t_{n}\right) .
\end{aligned}
$$

Therefore,

$$
\begin{aligned}
\operatorname{pr}_{N} \alpha_{n-1}\left(0, t_{2}, \ldots, t_{n}\right) & =\operatorname{pr}_{N} \partial^{0} \eta_{n-1}\left(t_{2}, \ldots, t_{n}\right), \\
\operatorname{pr}_{N} \alpha_{n-1}\left(t_{1}, \ldots, t_{i-1}, 0, t_{i+1}, \ldots, t_{n}\right) & =0 \quad \text { for } i>1, \\
\operatorname{pr}_{N} \alpha_{n-1}\left(t_{1}, \ldots, t_{i-1}, 1, t_{i+1}, \ldots, t_{n}\right) & =0
\end{aligned}
$$

since all other terms lie in $\left(M^{\prime}\right)^{n+1}$ (the span of $\left\{\partial^{i}: i>0\right\}$ ).

Since $\operatorname{pr}_{N} \partial^{0}=\operatorname{pr}_{N} d_{c}$, it follows from [Wei, Lemma 8.3.7] that on $N_{c}^{n}$,

$$
\operatorname{pr}_{N} \partial^{0}=d_{c}=\sum_{i=0}^{n+1}(-1)^{i} \partial^{i} .
$$

This implies that

$$
\eta_{n} \in N_{c}^{n+1} \mathrm{C}(E)^{I^{n} / \beth^{n}}
$$

and that the condition for $\eta_{n}$ to lift $\operatorname{pr}_{N}\left(\alpha_{n-1}\right)$ is precisely that $\delta\left(\eta_{n}\right)=d_{c} \eta_{n-1}$.

Corollary 4.9. A representative for $\mathbf{L} \cot (\iota \bullet)$ is given by $\iota(\mathbb{Z}) \cong \mathcal{E} D_{c}\left(\mathbb{Z}^{[-1]}\right)$, for

$$
\mathcal{E}: c s \mathrm{Ab} \rightarrow Q M^{*}(s \mathrm{Ab}, \times)
$$

as in Lemma 4.1.

Proof. First observe that, for $\Xi$ from Definition 3.10,

$$
\mathbf{L} \cot (\iota \bullet) \simeq \mathbf{L} \cot (\Xi)
$$

since $\Xi \rightarrow \iota \bullet$ is a cofibrant replacement.

Since $\Xi$ represents $\underline{\mathrm{MC}}$, for any simplicial cochain complex $V$ (in non-negative cochain degrees) we have $\operatorname{Hom}\left(N_{c} \mathrm{C}(\cot \Xi), V\right) \cong \mathrm{MC}\left(\mathcal{E}\left(D_{c} V\right)\right)$, where Hom is taken in the category of simplicial cochain complexes. Since $\mathrm{C}$ is inverse to $\mathcal{E}$, and $N_{c}$ is 
inverse to $D_{c}$, Proposition 4.8 can then be rephrased to say that

$$
N_{c}^{n} \mathrm{C}(\cot (\Xi)) \cong \begin{cases}\mathbb{Z}\left(I^{n-1} / \beth^{n-1}\right) & n \geqslant 1 \\ 0 & n=0\end{cases}
$$

with differential $d_{c}=\delta$.

Thus the bicomplex $N^{s} N_{c} \mathrm{C}(\cot \Xi)$ is weakly equivalent (in the Reedy model category of cochain diagrams in chain complexes) to the bicomplex $\mathbb{Z}^{[-1]}$, consisting of $\mathbb{Z}$ concentrated in cochain degree 1 and chain degree 0 . This means that cot $\Xi$ is weakly equivalent to $\mathcal{E} D_{c}\left(\mathbb{Z}^{[-1]}\right)$ (with constant simplicial structure), but this is isomorphic to $\iota(\mathbb{Z})$ (having $n$ copies of $\mathbb{Z}$ in level $n$ ).

Definition 4.10. Given a cochain complex $V$, denote the brutal truncation in degrees $\geqslant n$ by $\sigma^{\geqslant n} V$, so

$$
\left(\sigma^{\geqslant n} V\right)^{i}= \begin{cases}V^{i} & i \geqslant n \\ 0 & i<n .\end{cases}
$$

Definition 4.11. Define the total complex functor Tot $^{\Pi}$ from chain cochain complexes (i.e. bicomplexes) to chain complexes by

$$
\left(\operatorname{Tot}^{\Pi} V\right)_{n}:=\prod_{a-b=n} V_{a}^{b}
$$

with differential $d:=d^{s}+(-1)^{a} d_{c}$ on $V_{a}^{b}$.

Proposition 4.12. For $A \in \operatorname{cs} \mathrm{Ab}, \pi_{n} \underline{\mathrm{MC}}(A) \cong \mathrm{H}_{n-1}\left(\operatorname{Tot}^{\Pi} \sigma^{\geqslant 1} N^{s} N_{c} A\right)$.

Proof. First, note that $\underline{\mathrm{MC}}(A)=\underline{\operatorname{Hom}}_{Q M^{*}(\mathbb{S})}(\Xi, A)$, so the Dold-Kan correspondences give

$$
\underline{\mathrm{MC}}(A)=\underline{\operatorname{Hom}}_{c s \mathrm{Ab}}(\cot \Xi, A) \simeq \underline{\operatorname{Hom}}_{D G \geqslant 0} d g_{\geqslant 0} \mathrm{Ab}\left(N^{s} N_{c} \mathrm{C}(\cot \Xi), N^{s} N_{c} A\right),
$$

where $D G^{\geqslant 0} d g_{\geqslant 0} \mathrm{Ab}$ is the Reedy category of non-negatively graded cochain complexes of non-negatively graded chain complexes.

Now, Corollary 4.9 implies that $N^{s} N_{c} \mathrm{C}(\cot \Xi)$ is a cofibrant replacement for $\mathbb{Z}^{[-1]}$ in $D G^{\geqslant 0} d g_{00} \mathrm{Ab}$, so

$$
\underline{\mathrm{MC}}(A) \simeq \underline{\mathbf{R o m}}_{D G \geqslant 0} d g_{\geqslant 0} \mathrm{Ab}\left(\mathbb{Z}^{[-1]}, N^{s} N_{c} A\right) .
$$

A simpler cofibrant replacement is the object $L$ given by

$$
L_{i}^{n}= \begin{cases}\mathbb{Z} & n=i+1, i+2 \\ 0 & \text { otherwise }\end{cases}
$$

with chain and cochain differentials the identity whenever possible. Thus

$$
\underline{\mathrm{MC}}(A) \simeq \underline{\operatorname{Hom}}_{D G \geqslant 0} d g_{\geqslant 0} \mathrm{Ab}\left(L, N^{s} N_{c} A\right) .
$$

Now, a map $L \rightarrow B$ is determined by the images $b_{n}$ of the elements $1 \in L_{n-1}^{n}$, subject to the conditions that $d_{c} b_{n}=d^{s} b_{n+1}$. Thus

$$
\operatorname{Hom}_{D G \geqslant 0} d g_{\geqslant 0} \mathrm{Ab}(L, B) \cong \mathrm{Z}_{-1}\left(\operatorname{Tot}^{\Pi} \sigma^{\geqslant 1} B\right),
$$

where $\mathrm{Z}_{i}(V)=\operatorname{ker}\left(d: V_{i} \rightarrow V_{i-1}\right)$, and the description of $\pi_{n} \underline{\mathrm{MC}}(A)$ follows. 
Proposition 4.13. For $A \in c s \mathrm{Ab}$,

$$
\pi_{n} \operatorname{Del}_{A} \cong \mathrm{H}_{n-1}\left(\operatorname{Tot}^{\Pi} N^{s} N_{c} A\right) .
$$

Proof. We need to understand the adjoint action of $A^{0}$ on $\underline{\mathrm{MC}}(A)$. For $g \in A^{0}$ and $\omega \in \underline{\mathrm{MC}}(A)$, we have $g^{-1} * \omega * g=g^{-1} * 0 * g+\omega$, so the problem reduces to understanding the morphism $D: A^{0} \rightarrow \underline{\mathrm{MC}}(A)$ given by $D(g)=g^{-1} * 0 * g$.

If we now consider the simplicial normalisation of $\left[X /{ }^{h} G\right]$, for $X$ and $G$ abelian and $\left[-/^{h}-\right]$ as in Definition 3.14, we see that

$$
\begin{aligned}
N_{n}^{s}\left[X /{ }^{h} G\right] & \cong N_{n}^{s} X \oplus N_{n-1}^{s} G \\
\left(x, g, d^{s} g, 0,0, \ldots, 0\right) & \leftrightarrow(x, g) .
\end{aligned}
$$

The corresponding differential is given by $d^{s}(x, g)=\left(d^{s} x+g \cdot 0, d g\right)$, so $N^{s}\left[X /{ }^{h} G\right]$ is isomorphic to the mapping cone of the morphism $N^{s} G \stackrel{\cdot 0}{\longrightarrow} N^{s} X$.

If $X=\underline{\mathrm{MC}}(A)$ and $G=A^{0}$, then the gauge action on 0 is given by $g \cdot 0=D g$, so $N^{s} \operatorname{Del}_{A}$ is isomorphic to the cone complex of the morphism

$$
N^{s} A^{0} \stackrel{N^{s} D}{\longrightarrow} N^{s} \underline{\mathrm{MC}}(A) .
$$

Now,

$$
\underline{\operatorname{Hom}}_{c s \mathrm{Ab}}(\iota(\mathbb{Z}), A) \cong \mathrm{Z}^{1} N_{c} A:=\operatorname{ker}\left(d_{c}: N_{c}^{1} A \rightarrow N_{c}^{2} A\right),
$$

with the map $\cot (\Xi) \rightarrow \iota(\mathbb{Z})$ corresponding to the inclusion $f: \mathrm{Z}^{1} N_{c} A \hookrightarrow \underline{\mathrm{MC}}(A)$ given by $f(a)_{n}=\left(\partial^{1}\right)^{n}(a) \in A^{n+1} \subset\left(A^{n+1}\right)^{I^{n}}$. The key observation is that this subset is closed under the adjoint action, so, in particular, $D: A^{0} \rightarrow \underline{\mathrm{MC}}(A)$ factors through $\mathrm{Z}^{1} N_{c} A$, via the map $d_{c}: A^{0} \rightarrow \mathrm{Z}^{1} N_{c} A$.

The map $L \rightarrow \mathbb{Z}^{[-1]}$ corresponds to the natural inclusion

$$
\left(\mathrm{Z}^{1} N_{c} A\right)_{n} \hookrightarrow \mathrm{Z}_{-1}\left(\operatorname{Tot}^{\Pi} \sigma^{\geqslant 1} N_{c} A^{\Delta^{n}}\right) .
$$

The proof of Proposition 4.12 gives an equivalence

$$
N^{s} \underline{\mathrm{MC}}(A) \simeq \tau_{\geqslant 0}\left(\operatorname{Tot}^{\Pi}\left(\sigma^{\geqslant 1} N_{c} A\right)[-1]\right)
$$

of chain complexes, where $\tau_{\geqslant 0}$ is good truncation in non-negative degrees; thus

$$
\tau_{\geqslant 0}(V[-1])_{n}= \begin{cases}V_{n-1} & n>0 \\ \mathrm{Z}_{-1} V & n=0 \\ 0 & n<0 .\end{cases}
$$

Since this equivalence preserves the image of $N^{s} \underline{\operatorname{Hom}}_{c s \mathrm{Ab}}(\iota(\mathbb{Z}), A)$, it also preserves the image of $A^{0}$, giving an equivalence between the cone complexes of

$$
N^{s} A^{0} \stackrel{N^{s} D}{\longrightarrow} N^{s} \underline{\mathrm{MC}}(A) \quad \text { and } \quad N^{s} A^{0} \stackrel{d_{c}}{\longrightarrow} \tau_{\geqslant 0}\left(\operatorname{Tot}^{\Pi}\left(\sigma^{\geqslant 1} N_{c} A\right)[-1]\right) .
$$

The latter cone complex is just

$$
\tau_{\geqslant 0}\left(\operatorname{Tot}^{\Pi}\left(N^{s} N_{c} A\right)[-1]\right)
$$

which has the required cohomology. 


\subsection{Cohomology}

Definition 4.14. Given $E \in Q M^{*}(\mathbb{S})$ and $A \in c \mathrm{Ab}$, define cohomology groups of $E$ with coefficients in $A$ by

$$
\mathrm{H}^{i}(E, A):=\operatorname{Hom}_{\mathrm{Ho}\left(Q M^{*}(\mathbb{S})\right)}\left(E, N_{s}^{-1} A_{[-i]}\right),
$$

where $N_{s}^{-1} A_{[-i]}$ is the simplicial abelian group whose simplicial normalisation has $A$ concentrated in chain degree $i$.

Proposition 4.15. For $A \in c \mathrm{Ab}$,

$$
\mathrm{H}^{i}(\iota \bullet, A) \cong \begin{cases}\mathrm{H}^{i+1}\left(N_{c} A\right) & i>0 \\ \mathrm{Z}^{1} N_{c} A & i=0 .\end{cases}
$$

Proof. By Proposition 3.11, $\mathrm{H}^{i}(\iota \bullet, A) \cong \pi_{0} \underline{\mathrm{MC}}\left(N_{s}^{-1} A_{[-i]}\right)$, which by Proposition 4.12 is isomorphic to $\mathrm{H}_{-1}\left(\operatorname{Tot}^{\Pi} \sigma^{\geqslant 1} N_{c} A_{[-i]}\right)$. Since $\left(\operatorname{Tot}^{\Pi} \sigma^{\geqslant 1} N_{c} A_{[-i]}\right)_{n}=\left(\sigma^{\geqslant 1} N_{c} A\right)^{i-n}$, this is just $\mathrm{H}^{i+1}\left(\sigma^{\geqslant 1} N_{c} A\right)$, as required.

\subsection{Groupoids}

Given $\Gamma \in Q M^{*}(\mathrm{Gpd}, \times)$ (or even in $Q M^{*}(\mathrm{Cat}, \times)$ ), we have $B \Gamma \in Q M^{*}(\mathbb{S})$, for $B: \operatorname{Gpd} \rightarrow \mathbb{S}$ the nerve functor, and we now seek to describe the set $\operatorname{MC}(B \Gamma)$ (and hence the space $\underline{\mathrm{MC}}(B \Gamma))$.

Proposition 4.16. For $\mathcal{C} \in Q M^{*}(\mathrm{Cat}, \times)$, the set $\mathrm{MC}(B \mathcal{C})$ is isomorphic to the set of pairs $(x, a)$, for $x \in \mathrm{Ob} \mathcal{C}^{1}, a \in \mathcal{C}^{2}\left(x * x, \partial^{1} x\right)$ satisfying the following conditions:

$$
\begin{aligned}
\sigma^{0} x & =1, \\
\sigma^{0} a=\sigma^{1} a & =\operatorname{id}_{x} \in \mathcal{C}^{1}(x, x), \\
\left(\partial^{2} a\right) \circ(x * a) & =\left(\partial^{1} a\right) \circ(a * x) \in \mathcal{C}^{3}\left(x * x * x, \partial^{2} \partial^{1} x\right),
\end{aligned}
$$

where $a * x:=a * \operatorname{id}_{x}$ and $x * a=\mathrm{id}_{x} * a$, for $\operatorname{id}_{x} \in \mathcal{C}^{1}(x, x)$ the identity morphism.

Proof. First, observe that

$$
\operatorname{MC}(B \mathcal{C})=\operatorname{Hom}_{Q M^{*}(\mathbb{S})}(\Xi, B \mathcal{C}) \cong \operatorname{Hom}_{Q M^{*}(\mathrm{Cat})}\left(\tau_{1} \Xi, \mathcal{C}\right),
$$

where $\tau_{1} \Xi$ is the fundamental category of $\Xi$ (in the sense of $[\mathbf{J T}, \S 1]$ ).

It is therefore equivalent to show that $\tau_{1} \Xi$ is the quasi-comonoid in categories generated by an object $\xi \in \Xi_{0}^{1}$ and a morphism $\alpha \in \tau_{1}\left(\Xi^{2}\right)\left(\xi * \xi, \partial^{1} \xi\right)$ satisfying the conditions for $(x, a)$ above.

Note that $\Xi_{0}$ is the free quasi-comonoid (in sets) generated by the unique element $\xi \in \Xi_{0}^{1}$, subject to the condition that $\sigma^{0} \xi=1$. Recall that $\Xi_{0}^{n}=[0,1]^{n-1}$ for $n \geqslant 1$. We may then describe $\tau_{1} \Xi^{n}$ as the category associated to the poset $[0,1]^{n-1}$. Thus $\tau_{1} \Xi^{2}$ has objects $\xi * \xi$ and $\partial^{1} \xi$ (corresponding to 0 and 1 in $[0,1]$ respectively), with a unique isomorphism $\alpha: \xi * \xi \rightarrow \partial^{1} \xi$. Since there is a unique morphism from $(0,0)$ to $(1,1)$ in $\tau_{1} \Xi^{3}$, the morphisms

$$
\left(\partial^{2} \alpha\right) \circ(\xi * \alpha),\left(\partial^{1} \alpha\right) \circ(\alpha * \xi) \in \mathcal{C}^{3}\left(\xi * \xi * \xi, \partial^{2} \partial^{1} \xi\right)
$$

must be equal.

It therefore only remains to show that $\tau_{1} \Xi$ is isomorphic to the quasi-comonoid $\mathcal{D}$ defined to have objects $\mathrm{Ob} \Xi$, with morphisms freely generated by $\alpha \in \mathcal{D}\left(\xi * \xi, \partial^{1} \xi\right)$, 
subject to the condition $\left(\partial^{2} \alpha\right) \circ(\xi * \alpha)=\left(\partial^{1} \alpha\right) \circ(\alpha * \xi)$. Since the condition is satisfied by $\tau_{1} \Xi$, there is a natural map $\mathcal{D} \rightarrow \tau_{1} \Xi$ in $Q M^{*}$ (Cat).

Now, for $u \in \mathcal{D}^{m}(x, y)$ and $v \in \mathcal{D}^{n}\left(x^{\prime}, y^{\prime}\right)$, the fact that $\mathcal{D}^{m} \times \mathcal{D}^{n} \stackrel{*}{\rightarrow} \mathcal{D}^{m+n}$ is a functor implies that

$$
\left(\operatorname{id}_{y} * v\right) \circ\left(u * \operatorname{id}_{x^{\prime}}\right)=u * v=\left(u * \operatorname{id}_{y^{\prime}}\right) \circ\left(\operatorname{id}_{x} * v\right) .
$$

Therefore every morphism in $\mathcal{D}$ can be generated from $\alpha$ by the operations $\partial^{i}, \xi *, * \xi$ and composition. Thus every morphism is a composition of morphisms of the form

$$
\partial^{i_{r}} \ldots \partial^{i_{1}}\left(\xi^{s} * \alpha * \xi^{t}\right)
$$

There are $2^{n-2}(n-1)$ such morphisms in $\mathcal{D}^{n}$, corresponding to edges in the $(n-1)$ cube $\mathrm{Ob} \mathcal{D}^{n}=[0,1]^{n-1}$, so we call these the edge morphisms. Since $\tau_{1} \Xi^{n}$ is generated by edge morphisms, this implies that the functor $\mathcal{D}^{n} \rightarrow \tau_{1} \Xi^{n}$ is full.

Finally, the condition $\left(\partial^{2} \alpha\right) \circ(\xi * \alpha)=\left(\partial^{1} \alpha\right) \circ(\alpha * \xi)$ implies that any square of edge morphisms in $\mathcal{D}^{n}$ commutes. Thus $\mathcal{D}^{n}$ is the category associated to the poset $[0,1]^{n-1}$, so $\mathcal{D} \rightarrow \tau_{1} \Xi$ is an isomorphism, as required.

Corollary 4.17. For $\Gamma \in Q M^{*}(\mathrm{Gpd})$,

$$
\operatorname{Hom}_{\mathrm{Ho}\left(Q M^{*}(\mathbb{S})\right)}(\iota \bullet, B \Gamma) \cong \mathrm{MC}(B \Gamma) / \sim,
$$

where for pairs $(x, a)$ as in Proposition 4.16, the equivalence relation $\sim$ is given by saying that $(x, a) \sim\left(x^{\prime}, a^{\prime}\right)$ if there exists $\lambda \in \Gamma^{1}\left(x, x^{\prime}\right)$ such that

$$
\left(\partial^{1} \lambda\right) \circ a=a^{\prime} \circ(\lambda * \lambda), \quad \sigma^{0} \lambda=\mathrm{id}_{1},
$$

where $\mathrm{id}_{1}$ is the identity morphism in $\Gamma^{0}(1,1)$.

Proof. Let $\operatorname{Ar}(\Gamma) \in Q M^{*}(\mathrm{Gpd})$ be the groupoid of arrows in $\Gamma$, defined levelwise, and observe that

$$
B \Gamma \stackrel{\mathrm{id}}{\longrightarrow} B \operatorname{Ar}(\Gamma) \rightarrow B(\Gamma \times \Gamma)
$$

is a path object for $B \Gamma$. Since $\Xi$ is a cofibrant replacement for $\iota \bullet$, this gives

$$
\operatorname{Hom}_{\operatorname{Ho}\left(Q M^{*}(\mathbb{S})\right)}(\iota \bullet, B \Gamma) \cong \operatorname{Hom}_{Q M^{*}(\mathbb{S})}(\Xi, B \Gamma) / \operatorname{Hom}_{Q M^{*}(\mathbb{S})}(\Xi, B \operatorname{Ar}(\Gamma)),
$$

which is just $\mathrm{MC}(B \Gamma) / \mathrm{MC}(B \operatorname{Ar}(\Gamma))$.

Applying Proposition 4.16 to $\operatorname{Ar}(\Gamma)$ shows that elements of $\operatorname{MC}(B \operatorname{Ar}(\Gamma))$ correspond to pairs

$$
\left(\begin{array}{cccc} 
& x * x \stackrel{\lambda * \lambda}{\longrightarrow} x^{\prime} * x^{\prime} \\
x \stackrel{\lambda}{\longrightarrow} x^{\prime}, \quad{ }^{\prime} \downarrow & & \downarrow^{\prime} \\
& \partial^{1} x \stackrel{\partial^{1} \lambda}{\longrightarrow} & \partial^{1} x^{\prime}
\end{array}\right),
$$

with $(x, a),\left(x^{\prime}, a^{\prime}\right) \in \operatorname{MC}(B \Gamma)$ and $\left(\partial^{1} \lambda\right) \circ a=a^{\prime} \circ(\lambda * \lambda), \sigma^{0} \lambda=\mathrm{id}_{1}$. This gives the required description.

\subsection{Linear quasi-comonoids}

Given $E \in Q M^{*}(\mathbb{S})$, we now wish to describe the homology groups $\mathrm{H}_{*}\left(E^{n}, \mathbb{Z}\right)$, for all $n$ - by definition, these are just homology groups of the simplicial abelian group 
$\mathbb{Z} \otimes E$ freely generated by $E$. Applying $\mathbb{Z} \otimes$ levelwise gives a functor

$$
\mathbb{Z} \otimes: Q M^{*}(\mathbb{S}) \rightarrow Q M^{*}(s \mathrm{Ab}, \otimes),
$$

where the latter category is not to be confused with the category $Q M^{*}(s \mathrm{Ab}, \times)$ considered earlier. There is a forgetful functor, right adjoint to $\mathbb{Z} \otimes$, and [Hir, Theorem 11.3.2] allows us to put a model structure on $Q M^{*}(s \mathrm{Ab}, \otimes)$ for which a morphism is a weak equivalence or a fibration if the underlying map in $Q M^{*}(\mathbb{S})$ is so.

This means that a morphism in $Q M^{*}(s \mathrm{Ab}, \otimes)$ is a weak equivalence or a fibration whenever the underlying map in the Reedy category $\mathbb{S}^{\Delta_{* *}}$ is so. Thus the forgetful functor $Q M^{*}(s \mathrm{Ab}, \otimes) \rightarrow(s \mathrm{Ab})^{\Delta_{* *}}$ is right Quillen, where the latter category has the Reedy model structure.

Alternatively, we can forget the operations $\partial^{i}, \sigma^{i}$ and retain the multiplication. This gives us a forgetful functor $U: Q M^{*}(\mathrm{Ab}, \otimes) \rightarrow G$ Ring to simplicial (not necessarily commutative) $\mathbb{N}_{0}$-graded rings with unit, given by $U(R)^{n}=R^{n}$.

Lemma 4.18. The forgetful functor $U: Q M^{*}(s \mathrm{Ab}, \otimes) \rightarrow s G \operatorname{Ring}$ is left Quillen.

Proof. We first note that the model structure which we will use for $s G$ Ring is defined by saying that $f: R \rightarrow S$ is a weak equivalence (resp. a fibration) whenever all the maps $f^{n}: R^{n} \rightarrow S^{n}$ are weak equivalences (resp. fibrations) in $\mathbb{S}$. That this defines a model structure follows from [Hir, Theorem 11.3.2].

We may explicitly describe the right adjoint $U_{*}$ by

$$
\left(U_{*} R\right)^{n}:=\prod_{\substack{m \in \mathbb{N}_{0} \\ f: \mathbf{n} \rightarrow \mathbf{m} \text { in } \Delta}} R^{m},
$$

where for any morphism $g: n \rightarrow n^{\prime}$ in $\Delta$, the map $g:\left(U_{*} R\right)^{n} \rightarrow\left(U_{*} R\right)^{n^{\prime}}$ is given by $g(r)_{f}=r_{g \circ f}$, for $f: \mathbf{n}^{\prime} \rightarrow \mathbf{m}$.

The matching maps of this all have canonical sections, so it follows immediately that $U_{*}$ preserves fibrations and trivial fibrations, hence is right Quillen.

Definition 4.19. Given a (not necessarily commutative) ring $R$, and an $R$-bimodule $M$, define the set $\operatorname{Der}(R, M)$ of derivations to be the set of ring homomorphisms $R \rightarrow R \oplus M \epsilon$ over $R$, where $\epsilon^{2}=0$ and $\epsilon$ commutes with everything. Equivalently, a derivation is a morphism $f: R \rightarrow M$ such that $f(a b)=a f(b)+f(a) b$.

Then (as in $[\mathbf{D V}]$ ) define the $R$-bimodule $\Omega(R)$ to be the kernel of the multiplication map $R \otimes R \rightarrow R$; this has the universal property that $\operatorname{Hom}_{R-R}(\Omega(R), M) \cong$ $\operatorname{Der}(R, M)$, with the universal derivation $R \rightarrow \Omega(R)$ given by $r \mapsto r \otimes 1-1 \otimes r$.

We now also denote the forgetful functor $Q M^{*}(\mathrm{Ab}, \times) \rightarrow G \mathrm{Ab}$ to $\mathbb{N}_{0}$-graded abelian groups by $U$.

Lemma 4.20. For $E \in Q M^{*}$ (Set), there is a natural isomorphism

$$
U \cot (E) \cong U(\mathbb{Z} \otimes \iota \bullet) \otimes_{U(\mathbb{Z} \otimes E)} \Omega(U(\mathbb{Z} \otimes E)) \otimes_{U(\mathbb{Z} \otimes E)} U(\mathbb{Z} \otimes \iota \bullet) .
$$

Proof. Given a graded $U(\mathbb{Z} \otimes \iota \bullet)$-bimodule $M$, we have

$$
\operatorname{Hom}_{U(\mathbb{Z} \otimes E)-U(\mathbb{Z} \otimes E)}(\Omega(U(\mathbb{Z} \otimes E)), M)=\operatorname{Der}(U(\mathbb{Z} \otimes E), M),
$$

making use of the augmentation $U(\mathbb{Z} \otimes E) \rightarrow U(\mathbb{Z} \otimes \iota \bullet)$ coming from the canonical map $E \rightarrow \iota \bullet$ to the final object. 
Now,

$$
\operatorname{Der}(U(\mathbb{Z} \otimes E), M)=\operatorname{Hom}_{G \operatorname{Ring} \downarrow U(\mathbb{Z} \otimes \iota \bullet)}(U(\mathbb{Z} \otimes E), U(\mathbb{Z} \otimes \iota \bullet) \oplus M \epsilon),
$$

for $\epsilon^{2}=0$, and this is isomorphic to $\operatorname{Hom}_{Q M^{*}(\operatorname{Set}) \downarrow U_{*} U(\mathbb{Z} \otimes \bullet \bullet)}\left(E, U_{*}(U(\mathbb{Z} \otimes \iota \bullet) \oplus M \epsilon)\right)$.

Now, observe that $c \mathrm{Ab}$ is equivalent to the category of $\mathbb{Z} \otimes \iota \bullet$-bimodules in $\mathrm{Ab}^{\Delta_{* *}}$, with operations $\partial^{0}$ and $\partial^{n+1}$ on level $n$ corresponding to left and right multiplication by the unique element of $(\iota \bullet)^{1}$. Given a $\mathbb{Z} \otimes \iota \bullet$-bimodule $N$ in $\mathrm{Ab}^{\Delta_{* *}}$, the equivalence $c \mathrm{Ab} \simeq Q M^{*}(\mathrm{Ab}, \times)$ then combines with the forgetful functor $U_{\mathrm{Ab}}: Q M^{*}(\mathrm{Ab}, \times) \rightarrow$ $Q M^{*}$ (Set) to give rise to an object of $Q M^{*}$ (Set). Explicitly, this is

$$
((\mathbb{Z} \otimes \iota \bullet) \oplus N \epsilon) \times(\mathbb{Z} \otimes \iota \bullet) \iota \bullet
$$

(noting that the underlying object in $\operatorname{Set}^{\Delta_{* *}}$ is just $N$ ).

Next, observe that the forgetful functor $\mathrm{Ab}^{\Delta_{* *}} \rightarrow G \mathrm{Ab}$ has right adjoint $U_{*}$, defined by the same formulae as the functor $U_{*}$ of Lemma 4.18. Thus we get $U_{*} M \in \mathrm{Ab}^{\Delta_{* *}}$, which has a natural $\mathbb{Z} \otimes \iota \bullet$-bimodule structure, allowing us to regard it as a cosimplicial complex. Moreover,

$$
\begin{aligned}
U_{\mathrm{Ab}} U_{*} M & =\left((\mathbb{Z} \otimes \iota \bullet) \oplus U_{*} M \epsilon\right) \times_{(\mathbb{Z} \otimes \iota \bullet)} \iota \bullet \\
& =\left(\left(U_{*} U(\mathbb{Z} \otimes \iota \bullet)\right) \oplus U_{*} M \epsilon\right) \times_{U_{*} U(\mathbb{Z} \otimes \iota \bullet} \iota \bullet \\
& =U_{*}(U(\mathbb{Z} \otimes \iota \bullet) \oplus M \epsilon) \times_{U_{*} U(\mathbb{Z} \otimes \iota \bullet)} \iota \bullet
\end{aligned}
$$

in $Q M^{*}$ (Set). Since cot is left adjoint to $U_{\mathrm{Ab}}$,

$$
\operatorname{Hom}_{c \mathrm{Ab}}\left(\cot E, U_{*} M\right) \cong \operatorname{Hom}_{Q M^{*}(\mathrm{Set})}\left(E, U_{*}(U(\mathbb{Z} \otimes \iota \bullet) \oplus M \epsilon) \times_{U_{*} U(\mathbb{Z} \otimes \bullet \bullet} \iota \bullet\right),
$$

which we have already seen is isomorphic to $\operatorname{Der}(U(\mathbb{Z} \otimes E), M)$.

Thus

$$
\begin{aligned}
\operatorname{Hom}_{U(\mathbb{Z} \otimes E)-U(\mathbb{Z} \otimes E)}(\Omega(U(\mathbb{Z} \otimes E)), M) & \cong \operatorname{Hom}_{c \mathrm{Ab}}\left(\cot E, U_{*} M\right) \\
& \cong \operatorname{Hom}_{U(\mathbb{Z} \otimes \bullet \bullet)-U(\mathbb{Z} \otimes \bullet \bullet)}(U \cot E, M),
\end{aligned}
$$

as required.

Corollary 4.21. The morphism $\mathbf{L} \cot \iota \bullet \simeq \cot (\iota \bullet)$ is a weak equivalence.

Proof. We could use our explicit cofibrant replacement $\Xi$ to calculate $\mathbf{L}$ cot, but instead we give an argument which will generalise more widely. Although $\iota \bullet$ is not cofibrant, the underlying graded ring $U(\mathbb{Z} \otimes \iota \bullet)$ is freely generated by the unique element of $(\iota \bullet)^{1}$, so it is cofibrant in $s G$ Ring. If we took a cofibrant replacement $E$ of $\iota \bullet$, we would then have a weak equivalence $U(\mathbb{Z} \otimes E) \rightarrow U(\mathbb{Z} \otimes \iota \bullet)$ of cofibrant objects. Therefore

$$
U(\mathbb{Z} \otimes \iota \bullet) \otimes_{U(\mathbb{Z} \otimes E)} \Omega(U(\mathbb{Z} \otimes E)) \otimes_{U(\mathbb{Z} \otimes E)} U(\mathbb{Z} \otimes \iota \bullet) \rightarrow \Omega(U(\mathbb{Z} \otimes \iota \bullet))
$$

would be a weak equivalence of simplicial $U(\mathbb{Z} \otimes \iota \bullet)$-modules, so Lemma 4.20 gives a weak equivalence $\cot (E) \rightarrow \cot (\iota \bullet)$, as required.

Proposition 4.22. Given a cofibrant object $R \in s G$ Ring, equipped with an augmentation $R \rightarrow \mathbb{Z}$, there is a spectral sequence

$$
F\left(\mathrm{H}_{*}\left(\mathbb{Z} \otimes_{R} \Omega(R) \otimes_{R} \mathbb{Z}\right)\right) \Longrightarrow \mathrm{H}_{*}(R),
$$

where $F$ is the free graded (non-commutative) $\mathbb{Z}$-algebra functor on a graded module. This converges whenever $R^{0}=\mathbb{Z}$. 
Proof. By [Qui2, §II.6], cofibrant simplicial rings are retracts of free simplicial rings, where a simplicial ring $R_{\bullet}$ is said to be free if there are free generators $C_{q} \subset R_{q}$ for all $q$, closed under the degeneracy operations of $R_{\bullet}$.

Since the proposition is unchanged by taking retractions, we may assume that $R$ is free. If $I=\operatorname{ker}(R \rightarrow \mathbb{Z})$ is the augmentation ideal, first observe that $R=\mathbb{Z} \oplus I$, and that freeness gives $\mathbb{Z} \otimes_{R} \Omega(R) \otimes_{R} \mathbb{Z}=I /(I \cdot I)$. There is a filtration on $R$ by powers of $I$, with associated graded algebra $\operatorname{gr}_{I} R=\bigoplus_{n \geqslant 0} I^{\cdot n} / I^{\cdot n+1}$. Since $R$ is free, the canonical map $F(I /(I \cdot I)) \rightarrow \operatorname{gr}_{I} R$ is an isomorphism. The spectral sequence of the proposition is then just the spectral sequence associated to this filtration.

Finally, if $R^{0}=\mathbb{Z}$, then $I^{0}=0$, so $I^{\cdot n+1} \cap R^{n}=0$. We may regard the spectral sequence as a direct sum of spectral sequences, using the graded decomposition. In degree $n$, this gives the spectral sequence associated to the filtration $I^{p p} \cap R^{n}=0$ of $R^{n}$. Since this filtration is bounded, the spectral sequence converges.

Corollary 4.23. If $f: E \rightarrow F$ in $Q M^{*}$ (Set) is a morphism with $E^{0}=F^{0}=1, \pi_{f} E \simeq$ $\pi_{f} F \simeq \bullet$ and $\mathbf{L} \cot (E) \simeq \mathbf{L} \cot (F)$, then $f$ is a weak equivalence. Moreover, if

$$
\mathbf{L} \cot (E) \simeq \cot (\iota \bullet)
$$

then $E \rightarrow \iota \bullet$ is a weak equivalence.

Proof. We may choose cofibrant replacements $\tilde{E}, \tilde{F}$ of $E, F$, with $\tilde{E}^{0}=\tilde{F}^{0}=1$. Since the objects $\tilde{E}^{n}, \tilde{F}^{n}$ are simply connected for all $n$, we only need to prove that the map $\mathrm{H}_{*}\left(\tilde{E}^{n}, \mathbb{Z}\right) \rightarrow \mathrm{H}_{*}\left(\tilde{F}^{n}, \mathbb{Z}\right)$ of homology groups is an isomorphism. Now, $\mathrm{H}_{*}\left(\tilde{E}^{n}, \mathbb{Z}\right)=$ $\mathrm{H}_{*}\left(\mathbb{Z} \otimes \tilde{E}^{n}\right)$, so we may apply Lemma 4.20 and Proposition 4.22 to give the required isomorphism.

For the final part, note that Corollary 4.21 gives

$$
\mathbf{L} \cot (\iota \bullet) \simeq \cot (\iota \bullet)
$$

\subsection{Diagonals}

In this section, we will study properties of the diagonal functor diag: $Q M M^{*}(\mathbb{S}) \rightarrow$ $Q M^{*}(\mathbb{S})$, with a view to characterising $\mathrm{MC}(\operatorname{diag} E)$, thereby extending Lemma 1.21 to the simplicial case.

\subsubsection{Groupoids}

Our first step is to establish a diagonal version of Proposition 4.16.

Definition 4.24. Given a $\Delta_{* *} \times \Delta_{* *}$-diagram $S^{\bullet}, \bullet$ of sets, and a distinguished point $1 \in S^{n-1, i}$, define $\left(N_{h}^{n} S\right)^{i}=S^{n, i} \cap \bigcap_{j} \operatorname{ker}\left(\sigma_{h}^{j}\right)$, where ker denotes the inverse image of 1. Similarly, given $1 \in S^{i, n-1}$, define $\left(N_{v}^{n} S\right)^{i}=S^{i, n} \cap \bigcap_{j} \operatorname{ker}\left(\sigma_{v}^{j}\right)$.

Lemma 4.25. Given $\Gamma \in Q M M^{*}(\mathrm{Gpd})$, the set $\mathrm{MC}(\operatorname{diag} B \Gamma)$ consists of the data $(x, s, t, a, b)$, where $x \in \mathrm{Ob} \Gamma^{11}$, and for $x_{h}:=\sigma_{v}^{0} x, x_{v}:=\sigma_{h}^{0} x$,

$$
s \in \Gamma^{20}\left(x_{h} * x_{h}, \partial_{h}^{1} x_{h}\right), \quad t \in \Gamma^{02}\left(x_{v} * x_{v}, \partial_{v}^{1} x_{v}\right)
$$

and

$$
a \in N_{h} N_{v} \Gamma^{11}\left(x_{h} * x_{v}, x\right), \quad b \in N_{h} N_{v} \Gamma^{11}\left(x_{v} * x_{h}, x\right) .
$$


These data satisfy the additional conditions that

$$
\left(x_{h}, s\right) \in \operatorname{MC}\left(B \Gamma^{\bullet 0}\right), \quad\left(x_{v}, t\right) \in \operatorname{MC}\left(B \Gamma^{0 \bullet}\right)
$$

(as in Lemma 4.16), and that if we set $\gamma=b^{-1} \circ a: x_{h} * x_{v} \rightarrow x_{v} * x_{h}$, then

$$
\begin{aligned}
\partial_{h}^{1} \gamma & =\left(x_{v} * s\right) \circ\left(\gamma * x_{h}\right) \circ\left(x_{h} * \gamma\right) \circ\left(s * x_{v}\right)^{-1} \\
\partial_{v}^{1} \gamma^{-1} & =\left(x_{h} * t\right) \circ\left(\gamma^{-1} * x_{v}\right) \circ\left(x_{v} * \gamma^{-1}\right) \circ\left(t * x_{h}\right)^{-1} .
\end{aligned}
$$

Proof (sketch). By Proposition 4.16, we know that $\mathrm{MC}(\operatorname{diag} B \Gamma)$ consists of pairs $(x, \alpha)$, with $x \in \mathrm{Ob} \Gamma^{11}, \alpha \in \Gamma^{22}\left(x * x, \partial_{h}^{1} \partial_{v}^{1} x\right)$, satisfying various conditions. If we look at $a:=\sigma_{v}^{0} \sigma_{h}^{1} \alpha, b:=\sigma_{h}^{0} \sigma_{v}^{1} \alpha$, then we have $a: \sigma_{v}^{0} x * \sigma_{h}^{0} x \rightarrow x$ and $b: \sigma_{h}^{0} x * \sigma_{v}^{0} x \rightarrow x$. We also set $s=\left(\sigma_{v}^{0}\right)^{2} \alpha \in \Gamma^{20}$ and $t=\left(\sigma_{h}^{0}\right)^{2} \alpha \in \Gamma^{02}$.

Note that

$$
\left(\sigma_{v}^{0} x, s\right) \in \operatorname{MC}\left(B \Gamma^{\bullet 0}\right), \quad\left(\sigma_{h}^{0} x, t\right) \in \operatorname{MC}\left(B \Gamma^{0 \bullet}\right), \quad a, b \in N_{h} N_{v} \Gamma^{11},
$$

by applying powers of $\sigma_{h}^{0}$ or $\sigma_{v}^{0}$ to the equations for $\alpha$.

Applying the operations $\sigma_{h}^{i} \sigma_{v}^{j} \sigma_{v}^{k}$ to the equation

$$
\left(\partial_{h}^{2} \partial_{v}^{2} \alpha\right) \circ(x * \alpha)=\left(\partial_{h}^{1} \partial_{v}^{1} \alpha\right) \circ(\alpha * x)
$$

for $\{i, j, k\}=\{1,2,3\}$ and $j<k$ gives us the following equations in $\sigma_{v}^{0} \alpha, \sigma_{v}^{1} \alpha$ :

$$
\begin{aligned}
\sigma_{v}^{1} \alpha & =\left(\partial_{h}^{1} b\right) \circ\left(b * x_{h}\right)^{-1} \\
\left(\sigma_{v}^{0} \alpha\right)^{-1} \circ\left(\sigma_{v}^{1} \alpha\right) & =\left(x_{h} * b\right) \circ\left(a * x_{h}\right)^{-1} \\
\sigma_{v}^{0} \alpha & =\left(\partial_{h}^{1} a\right) \circ\left(x_{h} * a\right)^{-1},
\end{aligned}
$$

which reduce to the first condition for $\gamma$. Interchanging horizontal and vertical structures does the same for $\sigma_{h}^{0} \alpha, \sigma_{h}^{1} \alpha$, giving the second condition (for $\gamma^{-1}$ ).

It remains to show that we can recover $\alpha$ from $s, t, a, b$. It is the composition

$$
\left(\partial_{h}^{1} \partial_{v}^{1} a\right) \circ(s * t) \circ\left(x_{h} * \gamma * x_{v}\right) \circ\left(a^{-1} * a^{-1}\right) .
$$

Proposition 4.26. For $\Gamma \in Q M M^{*}(\mathrm{Gpd})$, elements of $\operatorname{Hom}_{\operatorname{Ho}\left(Q M^{*}(\mathbb{S})\right)}(\iota \bullet, B \operatorname{diag} \Gamma)$ are represented by data of the form $\left(x_{h}, x_{v}, s, t, \gamma\right)$, where

$$
\left(x_{h}, s\right) \in \operatorname{MC}\left(B \Gamma^{\bullet, 0}\right), \quad\left(x_{v}, t\right) \in \operatorname{MC}\left(B \Gamma^{0, \bullet}\right),
$$

and $\gamma: x_{h} * x_{v} \rightarrow x_{v} * x_{h}$ satisfies the conditions of Lemma 4.25. Two systems

$$
\left(x_{h}, x_{v}, s, t, \gamma\right),\left(x_{h}^{\prime}, x_{v}^{\prime}, s^{\prime}, t^{\prime}, \gamma^{\prime}\right)
$$

represent the same element if and only if there exist

$$
\lambda_{h} \in \Gamma^{1,0}\left(x_{h}, x_{h}^{\prime}\right), \quad \lambda_{v} \in \Gamma^{0,1}\left(x_{v}, x_{v}^{\prime}\right)
$$

such that

$$
\begin{aligned}
\left(\partial_{h}^{1} \lambda_{h}\right) \circ s & =s^{\prime} \circ\left(\lambda_{h} * \lambda_{h}\right) \quad \sigma_{h}^{0} \lambda_{h}=1, \\
\left(\partial_{v}^{1} \lambda_{v}\right) \circ t & =t^{\prime} \circ\left(\lambda_{v} * \lambda_{v}\right) \quad \sigma_{v}^{0} \lambda_{v}=1, \\
\gamma^{\prime} \circ\left(\lambda_{h} * \lambda_{v}\right) & =\left(\lambda_{v} * \lambda_{h}\right) \circ \gamma .
\end{aligned}
$$


Proof (sketch). Take $(x, \alpha)$ as in the proof of Lemma 4.25. By Corollary 4.17, $(x, \alpha) \sim$ $\left(x^{\prime}, \alpha^{\prime}\right)$ whenever there exists $\lambda \in \Gamma^{11}\left(x, x^{\prime}\right)$ such that

$$
\left(\partial_{h}^{1} \partial_{v}^{1} \lambda\right) \circ \alpha=\alpha^{\prime} \circ(\lambda * \lambda) \quad \sigma_{h}^{0} \sigma_{v}^{0} \lambda=1 .
$$

Given $(x, \alpha)$, we may therefore set

$$
x^{\prime}=x_{h} * x_{v}, \quad \alpha^{\prime}=\left(\partial_{h}^{1} \partial_{v}^{1} a^{-1}\right) \circ \alpha \circ(a * a)
$$

and define a transformation $\lambda:(x, \alpha) \rightarrow\left(x^{\prime}, \alpha^{\prime}\right)$ by $\lambda=a^{-1}$.

Therefore, every element of $\operatorname{Hom}_{\operatorname{Ho}\left(Q M^{*}(\mathbb{S})\right)}(\iota \bullet, B \operatorname{diag} \Gamma)$ has a representative with $x=x_{h} * x_{v}$, and $a=1$, giving data $\left(x_{h}, x_{v}, s, t, \gamma\right)$ as above. Two such systems are equivalent if there exists a transformation $\lambda \in \Gamma^{11}\left(x_{h} * x_{v}, x_{h}^{\prime} * x_{v}^{\prime}\right)$ satisfying the conditions of Lemma 4.17. Since $a=1$, we recover that $\lambda=\lambda_{h} * \lambda_{v}$ for $\lambda_{h}:=\sigma_{v}^{0} \lambda$ and $\lambda_{v}:=\sigma_{h}^{0} \lambda$. The conditions for $\lambda$ then reduce to the conditions for $\lambda_{h}, \lambda_{v}$ above.

Corollary 4.27. The object $\operatorname{diag}^{*}(\Xi) \in Q M M^{*}(\mathbb{S})$ is simply connected in every level.

Proof. By Lemma 4.25, the fundamental groupoid $\Gamma:=\pi_{f}\left(\operatorname{diag}^{*} \Xi\right) \in Q M M^{*}(\mathrm{Gpd})$ is generated by an object $x \in \mathrm{Ob} \Gamma^{1,1}$, together with isomorphisms $(s, t, a, b)$ satisfying the conditions of that lemma. Consider $\Upsilon \in Q M M^{*}(\mathrm{Gpd})$ generated by objects

$$
x_{h} \in \mathrm{Ob} \Gamma^{1,0}, \quad x_{v} \in \mathrm{Ob} \Gamma^{0,1}
$$

and isomorphisms $(s, t, \gamma)$ satisfying the conditions of Lemma 4.26. That lemma implies that the canonical inclusion $\Upsilon \rightarrow \pi_{f} \operatorname{diag}^{*} \Xi$ is a levelwise equivalence.

Now, the objects of $\Upsilon$ are words in $\left(\partial_{h}^{1}\right)^{i} x_{h},\left(\partial_{v}^{1}\right)^{j} x_{v}$, and the conditions on $(s, t, a, b)$ ensure that there is a unique isomorphism between any two such words in the same level, so $\Upsilon^{m n}$ is simply connected. Thus the maps $\Upsilon \rightarrow \pi_{f} \operatorname{diag}^{*} \Xi \rightarrow \iota(\bullet, \bullet)$ are equivalences in every level, as required.

4.5.2. Abelian groups

Lemma 4.28. The category $Q M M^{*}(\mathrm{Ab}, \times)$ is equivalent to the category ccAb of bicosimplicial abelian groups.

Proof. The proof of Lemma 4.1 carries over to this context.

Definition 4.29. Define cot: $Q M M^{*}(\mathrm{Set}) \rightarrow Q M M^{*}(\mathrm{Ab}, \times) \simeq c c \mathrm{Ab}$ to be left adjoint to the forgetful functor $Q M M^{*}(\mathrm{Ab}, \times) \rightarrow Q M M^{*}$ (Set).

The following results have the same proofs as Corollary 4.21, Proposition 4.22 and Corollary 4.23, replacing the category GRing of graded rings with the category GGRing of bigraded rings.

Lemma 4.30. The morphism $\mathbf{L} \cot \iota(\bullet, \bullet) \rightarrow \cot \iota(\bullet, \bullet)$ is a weak equivalence.

Proposition 4.31. Given a cofibrant object $R \in s G G \mathrm{Ring}$, equipped with an augmentation $R \rightarrow \mathbb{Z}$, there is a spectral sequence

$$
F\left(\mathrm{H}_{*}\left(\mathbb{Z} \otimes_{R} \cot (R) \otimes_{R} \mathbb{Z}\right)\right) \Longrightarrow \mathrm{H}_{*}(R),
$$

where $F$ is the free bigraded (non-commutative) $\mathbb{Z}$-algebra functor on a bigraded module. This converges whenever $R^{00}=\mathbb{Z}$. 
Corollary 4.32. If $f: E \rightarrow F$ in $Q M M^{*}$ (Set) is a morphism with $E^{00}=F^{00}=1$, $\pi_{f} E \simeq \pi_{f} F \simeq \bullet$ and $\mathbf{L} \cot (E) \simeq \mathbf{L} \cot (F)$, then $f$ is a weak equivalence. Moreover, if $\mathbf{L} \cot (E) \simeq \cot \iota(\bullet, \bullet)$, then $E \rightarrow \iota(\bullet, \bullet)$ is a weak equivalence.

Proposition 4.33. The map $\mathbf{L} \operatorname{diag}^{*}(\iota \bullet) \rightarrow \iota(\bullet, \bullet)$ is a weak equivalence.

Proof. Proposition 4.26 shows that this gives an equivalence on fundamental groupoids, so by Corollary 4.32 it suffices to show that

$$
\mathbf{L} \cot \left(\mathbf{L} \operatorname{diag}^{*}(\iota \bullet)\right) \simeq \mathbf{L} \cot (\iota(\bullet, \bullet)) .
$$

Now the diagonal functor $\operatorname{diag}: Q M M^{*}(\mathrm{Ab}, \times) \rightarrow Q M^{*}(\mathrm{Ab}, \times)$ also has a left adjoint $\operatorname{diag}_{\mathrm{Ab}}^{*}: Q M^{*}(\mathrm{Ab}, \times) \rightarrow Q M M^{*}(\mathrm{Ab}, \times)$, which can be calculated in a similar way to the functor $d^{*}$ in $[\mathbf{G J}, \S$ IV.3.3], by studying the associated cosimplicial and bicosimplicial complexes. It follows from this description that $\operatorname{diag}_{\mathrm{Ab}}^{*}$ preserves weak equivalences. Moreover, the functors $\operatorname{diag}_{\mathrm{Ab}}^{*} \cot$ and $\cot \operatorname{diag}^{*}$ from $Q M^{*}$ (Set) to $Q M M^{*}(\mathrm{Ab}, \times)$ are naturally isomorphic, since their right adjoints are.

By Corollary $4.30, \mathbf{L} \cot \iota(\bullet, \bullet) \simeq \cot \iota(\bullet, \bullet)$, so it suffices to show that

$$
\operatorname{diag}_{\mathrm{Ab}}^{*} \cot \Xi \rightarrow \cot \iota(\bullet, \bullet)=\operatorname{diag}_{\mathrm{Ab}}^{*} \cot \iota \bullet
$$

is a weak equivalence. Since $\operatorname{diag}_{\mathrm{Ab}}^{*}$ preserves weak equivalences in $Q M^{*}(\mathrm{Ab}, \times)$, we need only observe that $\cot \Xi \rightarrow \cot \iota \bullet$ is a weak equivalence by Corollary 4.21 .

\section{Mapping spaces}

Lemma 5.1. For $\mathbf{n}$ as in Lemma 1.26, $P_{n}^{*} \operatorname{diag}^{*} \Xi$ is a cofibrant replacement for $\operatorname{alg}^{*} \mathbf{n}$

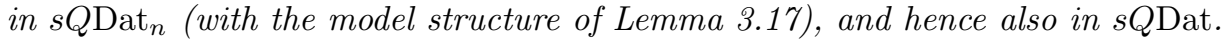

Proof. By Proposition 4.33, the morphism $\operatorname{diag}^{*} \Xi \rightarrow \iota(\bullet, \bullet)$ in $Q M M^{*}(\mathbb{S})$ is a weak equivalence. From the description of $P_{n}^{*}$ in Lemma 1.32, it follows that $P_{n}^{*}$ from Definition 3.23 preserves weak equivalences, so $P_{n}^{*} \operatorname{diag}^{*} \Xi \rightarrow P_{n}^{*} \iota(\bullet, \bullet)$ is also a weak equivalence. Since $P_{n}^{*}: Q M M^{*}(\mathbb{S}) \rightarrow s Q$ Dat $_{n}$ is left Quillen, $P_{n}^{*} \operatorname{diag}^{*} \Xi$ is cofibrant (and a realisation of $\left.P_{n}^{*} \mathbf{L} \operatorname{diag}^{*} \iota(\bullet)\right)$. Now we need only recall from the proof of Lemma 1.34 that $P_{n}^{*} \iota(\bullet, \bullet) \cong \operatorname{alg}^{*} \mathbf{n}$.

\section{1. $\mathcal{M C}$}

Proposition 5.2. For $\mathcal{D} \in s Q D$ at fibrant,

$$
\mathcal{M C}(\mathcal{D})_{n} \simeq \coprod_{f:[0, n] \rightarrow \mathrm{Ob} \mathcal{D}} \operatorname{Map}_{s Q \operatorname{Dat}_{n}}^{h}\left(\operatorname{alg}^{*} \mathbf{n}, f^{-1} \mathcal{D}\right)
$$

where $\mathrm{Map}^{h}$ denotes the derived function complex RMap of [Hov, §5].

Proof. For $\mathcal{E} \in s Q$ Dat $_{n}$,

$$
\underline{\mathrm{MC}}\left(\operatorname{diag} P_{n} \mathcal{E}\right)=\underline{\operatorname{Hom}}_{s Q \operatorname{Dat}_{n}}\left(P_{n}^{*} \operatorname{diag}^{*} \Xi, \mathcal{E}\right),
$$

recalling that (unlike $s Q$ Dat) the model category $s Q$ Dat $_{n}$ has a simplicial structure. Since $P_{n}^{*} \operatorname{diag}^{*} \Xi$ is cofibrant, this is equivalent to the derived function complex $\operatorname{Map}_{s Q \operatorname{Dat}_{n}}^{h}\left(P_{n}^{*} \operatorname{diag}^{*} \Xi, \mathcal{E}\right)$ whenever $\mathcal{E}$ is fibrant ([Hov, Theorem 5.4.9]). Since 
$P_{n}^{*} \operatorname{diag}^{*} \Xi$ is weakly equivalent to $\operatorname{alg}^{*} \mathbf{n}$, this function complex is also weakly equivalent to $\operatorname{Map}_{s Q \operatorname{Dat}_{n}}^{h}\left(\operatorname{alg}^{*} \mathbf{n}, \mathcal{E}\right)$. The description now follows immediately from Definition 3.25 .

Corollary 5.3. For $\mathcal{D} \in s Q D a t$ fibrant,

$$
\mathcal{M C}(\mathcal{D})_{n} \simeq \operatorname{Map}_{s Q \text { Dat }}^{h}\left(\operatorname{alg}^{*} \mathbf{n}, \mathcal{D}\right) \times_{\operatorname{Map}_{s \mathrm{Cat}}^{h}\left(\mathbf{0}, \mathcal{D}^{0}\right)^{[0, n]}}^{h}(\mathrm{Ob} \mathcal{D})^{[0, n]} .
$$

Proof. Comparing the model structures of Lemma 3.17 and Proposition 3.19, it follows immediately that the functor $\mathcal{O} \downarrow s Q$ Dat $\rightarrow s Q$ Dat $_{\mathcal{O}}$, given by mapping $f: \mathcal{O} \rightarrow \mathcal{D}$ to $f^{-1} \mathcal{D}$, preserves (trivial) fibrations. Its left adjoint is the inclusion functor $s Q$ Dat $_{\mathcal{O}} \rightarrow$ $\mathcal{O} \downarrow s Q D$ at, so these form a Quillen pair. Hence

$$
\begin{aligned}
\operatorname{Map}_{s Q \operatorname{Dat}_{n}}^{h}\left(\operatorname{alg}^{*} \mathbf{n}, f^{-1} \mathcal{D}\right) & \simeq \operatorname{Map}_{[0, n] \downarrow s Q \operatorname{Dat}^{h}}^{h}\left(\operatorname{alg}^{*} \mathbf{n},[0, n] \stackrel{f}{\rightarrow} \mathcal{D}\right) \\
& =\operatorname{Map}_{s Q \text { Dat }}^{h}\left(\operatorname{alg}^{*} \mathbf{n}, \mathcal{D}\right) \times_{\operatorname{Map}_{s Q \text { Dat }}^{h}([0, n], \mathcal{D})}^{h}\{f\} .
\end{aligned}
$$

Thus we have that $\mathcal{M C}(\mathcal{D})_{n}=\operatorname{Map}^{h}\left(\operatorname{alg}^{*} \mathbf{n}, \mathcal{D}\right) \times_{\operatorname{Map}^{h}([0, n], \mathcal{D})}^{h} \operatorname{Hom}([0, n], \mathcal{D})$, and

$$
\operatorname{Map}_{s Q \text { Dat }}^{h}([0, n], \mathcal{D})=\operatorname{Map}_{s \text { Cat }}^{h}\left([0 . n], \mathcal{D}^{0}\right)=\operatorname{Map}_{s \text { Cat }}^{h}\left(\mathbf{0}, \mathcal{D}^{0}\right)^{[0, n]} \text {. }
$$

Definition 5.4. For $n \in \mathbb{N}_{0}$, define $\Xi \times \operatorname{alg}^{*} \mathbf{n} \in s Q D$ at to have objects $[0, n]$ and morphisms $\left(\Xi \times \operatorname{alg}^{*} \mathbf{n}\right)(i, j)=\Xi$ for $i \leqslant j$ and $\emptyset$ otherwise. This can be characterised as the coproduct $\left(\Xi \times \operatorname{alg}^{*}(\mathrm{Ob} \mathbf{n})\right) \sqcup_{(\mathrm{id} / \emptyset)[0, n]}(\mathrm{id} / \emptyset)(\mathbf{n})$ in the category $s Q D a t$, or equivalently in the category $s Q \operatorname{Dat}_{n}$.

Lemma 5.5. The natural morphism $f: \Xi \times \operatorname{alg}^{*} \mathbf{n} \rightarrow P_{n}^{*} \operatorname{diag}^{*} \Xi$ is a trivial cofibration in $s Q$ Dat $_{n}$.

Proof. For $\mathcal{D} \in s Q$ Dat $_{n}$,

$$
\operatorname{Hom}_{s Q \operatorname{Dat}_{n}}\left(\Xi \times \operatorname{alg}^{*} \mathbf{n}, \mathcal{D}\right)=\operatorname{MC}\left(\left(P_{n} \mathcal{D}\right)^{\bullet, 0} \times\left(P_{n} \mathcal{D}\right)^{0,0}\left(P_{n} \mathcal{D}\right)^{0, \bullet}\right) .
$$

The morphism $f$ then corresponds to the map

$$
\left(\left(\sigma_{v}^{0}\right)^{\bullet},\left(\sigma_{h}^{0}\right)^{\bullet}\right): \operatorname{diag}\left(P_{n} \mathcal{D}\right) \rightarrow\left(P_{n} \mathcal{D}\right)^{\bullet, 0} \times{ }_{\left(P_{n} \mathcal{D}\right)^{0,0}}\left(P_{n} \mathcal{D}\right)^{0, \bullet}
$$

in $Q M^{*}(\mathbb{S})$.

Given a trivial fibration $\mathcal{D} \rightarrow \mathcal{E}$ in $s Q$ Dat $_{n}$, observe that the conditions (W1) and (F1) from Proposition 3.19 ensure that

$$
\operatorname{diag}\left(P_{n} \mathcal{D}\right) \rightarrow \operatorname{diag}\left(P_{n} \mathcal{E}\right) \times{ }_{\left(P_{n} \mathcal{E}\right)^{\bullet, 0}} \times_{\left(P_{n} \mathcal{E}\right)^{0,0}}\left(P_{n} \mathcal{E}\right)^{0, \bullet}\left(P_{n} \mathcal{D}\right)^{\bullet, 0} \times{ }_{\left(P_{n} \mathcal{D}\right)^{0,0}}\left(P_{n} \mathcal{D}\right)^{0, \bullet}
$$

is a trivial fibration in $Q M^{*}(\mathbb{S})$, so Lemma 3.9 implies that the functor MC applied to this map is surjective. Therefore $f$ is a cofibration.

Now, $\Xi \times \operatorname{alg}^{*} \mathbf{n} \simeq(\iota \bullet) \times \operatorname{alg}^{*} \mathbf{n}=\operatorname{alg}^{*} \mathbf{n}$ and $P_{n}^{*} \operatorname{diag}^{*} \Xi \simeq \operatorname{alg}^{*} \mathbf{n}$ by Lemma 5.1. Thus $f$ is a trivial cofibration in $s Q$ Dat $_{n}$.

Definition 5.6. Recall from [Rez, 4.1] that a Segal space is defined to be a bisimplicial set $W \in s \mathbb{S}$ which is Reedy fibrant, and for which the natural maps

$$
W_{k} \rightarrow \overbrace{W_{1} \times \partial_{0}, W_{0} \partial_{1} \cdots \times \partial_{0}, W_{0}, \partial_{1} W_{1}}^{k}
$$

are weak equivalences in $\mathbb{S}$ for all $k$. 
Proposition 5.7. For $\mathcal{D} \in s Q D$ at fibrant, $\mathcal{M C}(\mathcal{D})$ is a Segal space.

Proof. By applying Lemma 3.28 to the morphism $\mathcal{D} \rightarrow \operatorname{alg}^{*} \mathbf{0}$, we know that $\mathcal{M C}(\mathcal{D})$ is Reedy fibrant, since $\operatorname{cosk}_{0}(\mathrm{Ob} \mathcal{D})^{\mathrm{hor}}$ is Reedy fibrant, and $\mathcal{M C}\left(\operatorname{alg}^{*} \mathbf{0}\right)=\boldsymbol{\bullet}$.

Letting $W:=\mathcal{M C}(\mathcal{D})$, we have

$$
\begin{aligned}
\left(W_{n}\right)_{i} & =\coprod_{f:[0, n] \rightarrow \mathrm{Ob} \mathcal{D}} \underline{\operatorname{Hom}}_{s Q \operatorname{Dat}_{n}}\left(P_{n}^{*} \operatorname{diag}^{*} \Xi, f^{-1} \mathcal{D}\right)_{i} \\
& =\coprod_{f:[0, n] \rightarrow \mathrm{Ob} \mathcal{D}} \operatorname{Hom}_{s Q \operatorname{Dat}_{n}}\left(\left(P_{n}^{*} \operatorname{diag}^{*} \Xi\right),\left(f^{-1} \mathcal{D}\right)^{\Delta^{i}}\right) \\
& =\operatorname{Hom}_{s Q \operatorname{Dat}}\left(\left(P_{n}^{*} \operatorname{diag}^{*} \Xi\right), \mathcal{D}^{\Delta^{i}}\right),
\end{aligned}
$$

where $\mathcal{D}^{K}$ is defined by $\mathrm{Ob} \mathcal{D}^{K}=\mathrm{Ob} \mathcal{D}$ and $\underline{\mathcal{H} o m}_{\mathcal{D}^{K}}(a, b):=\underline{\mathcal{H} o m}_{\mathcal{D}}(a, b)^{K}$ (note that although this makes $s Q$ Dat into a simplicial category, it does not satisfy axiom (SM7) of a simplicial model category).

Now,

$$
\begin{aligned}
& \overbrace{\left(W_{1} \times \partial_{0}, W_{0}, \partial_{1} \cdots \times \partial_{0}, W_{0}, \partial_{1} W_{1}\right.}^{k})_{i} \\
& \cong \operatorname{Hom}_{s Q \operatorname{Dat}}\left(\left(P_{1}^{*} \operatorname{diag}^{*} \Xi\right) \cup_{P_{0}^{*} \Xi} \cdots \cup_{P_{0}^{*} \Xi}\left(P_{1}^{*} \operatorname{diag}^{*} \Xi\right), \mathcal{D}^{\Delta^{i}}\right) \\
& \cong \coprod_{f:[0, n] \rightarrow \mathrm{Ob} \mathcal{D}} \underline{\operatorname{Hom}}_{s Q \operatorname{Dat}_{n}}\left(\left(P_{1}^{*} \operatorname{diag}^{*} \Xi\right) \cup_{\Xi} \cdots \cup_{\Xi}\left(P_{1}^{*} \operatorname{diag}^{*} \Xi\right), f^{-1} \mathcal{D}\right)_{i},
\end{aligned}
$$

since $[0,1] \cup_{\{1\}}[1,2] \cup_{\{2\}} \cdots \cup_{\{n-1\}}[n-1, n]=[0, n]$.

Now, Lemma 5.5 implies that

$$
\left(\Xi \times \operatorname{alg}^{*} \mathbf{1}\right) \cup_{\Xi} \cdots \cup_{\Xi}\left(\Xi \times \operatorname{alg}^{*} \mathbf{1}\right) \rightarrow P_{1}^{*} \operatorname{diag}^{*} \Xi \cup_{\Xi} \cdots \cup_{\Xi} P_{1}^{*} \operatorname{diag}^{*} \Xi
$$

is a trivial cofibration (being a pushout of trivial cofibrations), and the left-hand side is just $\Xi \times \operatorname{alg}^{*}\left(\mathbf{1} \cup_{\mathbf{0}} \cdots \cup_{\mathbf{0}} \mathbf{1}\right)=\Xi \times \operatorname{alg}^{*} \mathbf{k}$. This is weakly equivalent to $\operatorname{alg}^{*} \mathbf{k}$, so

$$
\overbrace{W_{1} \times \partial_{\partial_{0}, W_{0}, \partial_{1}} \times \cdots \times{ }_{\partial_{0}, W_{0}, \partial_{1}} W_{1}}^{k} \simeq \coprod_{f:[0, n] \rightarrow \mathrm{Ob} \mathcal{D}} \operatorname{Map}_{s Q \operatorname{Dat}_{n}}^{h}\left(\operatorname{alg}^{*} \mathbf{k}, f^{-1} \mathcal{D}\right),
$$

which is equivalent to $W_{k}$ by Proposition 5.2.

\subsubsection{Morphism spaces}

Lemma 5.8. Given $\mathcal{D} \in Q \mathrm{Dat}$, and $x, y \in \mathrm{Ob} \operatorname{alg} \mathcal{D}$, the object $\mathcal{D}(\bar{x}, \bar{y}) \in \operatorname{Set}^{\Delta_{* *}}$ has the natural structure of a cosimplicial set, where $\bar{x}, \bar{y} \in \mathrm{Ob} \mathcal{D}$ are the objects underlying $x, y$.

Proof. The objects $x, y$ correspond to the elements

$$
\omega_{x} \in \operatorname{MC}(\mathcal{D}(x, x)), \quad \omega_{y} \in \operatorname{MC}(\mathcal{D}(y, y)) .
$$

In order to enhance the structure of $\mathcal{D}(\bar{x}, \bar{y})$, we define operations $\partial^{0}:=\omega_{x} *: \mathcal{D}(x, y)^{n}$ $\rightarrow \mathcal{D}(x, y)^{n+1}$, and $\partial^{n+1}:=* \omega_{y}: \mathcal{D}(x, y)^{n} \rightarrow \mathcal{D}(x, y)^{n+1}$. The Maurer-Cartan equations ensure that these operations satisfy the necessary conditions for a cosimplicial set. 
Definition 5.9. Recall from [GJ, $\S$ VIII.1] that the functor Tot: $c \mathbb{S} \rightarrow \mathbb{S}$ from cosimplicial simplicial sets to simplicial sets is given by

$$
\operatorname{Tot} X^{\bullet}=\left\{x \in \prod_{n}\left(X^{n}\right)^{\Delta^{n}}: \partial_{X}^{i} x_{n}=\left(\partial_{\Delta}^{i}\right)^{*} x_{n+1}, \sigma_{X}^{i} x_{n}=\left(\sigma_{\Delta}^{i}\right)^{*} x_{n-1}\right\} .
$$

When $X$ is Reedy fibrant, homotopy groups of the total space are related to homotopy groups of the spaces $X^{n}$ via a spectral sequence given in [GJ, Proposition VIII.1.15].

Proposition 5.10. Given $\mathcal{D} \in s Q D$ at fibrant, and $x, y \in \mathrm{Ob} \operatorname{alg}\left(\mathcal{D}_{0}\right)$, there is a natural weak equivalence

$$
\mathcal{M C}(\mathcal{D})_{1} \times_{\mathrm{MC}(\mathcal{D}) \times \operatorname{MC}(\mathcal{D})}\{(x, y)\} \simeq \operatorname{Tot} \mathcal{D}(\bar{x}, \bar{y}) .
$$

Proof. Define $f:[0,1] \rightarrow$ Ob $\mathcal{D}$ by $f(0)=\bar{x}, f(1)=\bar{y}$, and set $\mathcal{E}:=f^{-1} \mathcal{D} \in s Q$ Dat $_{1}$ (for $s Q \mathrm{Dat}_{n}$ as in Lemma 1.32). Then

$$
\begin{aligned}
& \mathcal{M C}(\mathcal{D})_{1} \times_{\mathrm{MC}(\mathcal{D}) \times \mathrm{MC}(\mathcal{D})}\{(x, y)\}= \underline{\operatorname{Hom}}_{(\Xi \sqcup \Xi) \downarrow s Q \operatorname{Dat}_{1}}\left(P_{1}^{*} \operatorname{diag}^{*} \Xi, \mathcal{E}\right) \\
&= \underline{\operatorname{Hom}}_{\operatorname{alg}^{*}(\mathbf{0} \sqcup \mathbf{0}) \downarrow s Q \operatorname{Dat}_{1}}\left(\left(P_{1}^{*} \operatorname{diag}^{*} \Xi\right)\right. \\
&\left.\cup_{(\Xi \sqcup \Xi)} \operatorname{alg}^{*}(\mathbf{0} \sqcup \mathbf{0}), \mathcal{E}\right) .
\end{aligned}
$$

By Lemma 5.5,

$$
\left(P_{1}^{*} \operatorname{diag}^{*} \Xi\right) \cup_{(\Xi \sqcup \Xi)} \operatorname{alg}^{*}(\mathbf{0} \sqcup \mathbf{0}) \simeq\left(\Xi \times \operatorname{alg}^{*} \mathbf{1}\right) \cup_{(\Xi \sqcup \Xi)} \operatorname{alg}^{*}(\mathbf{0} \sqcup \mathbf{0})=\operatorname{alg}^{*} \mathbf{1},
$$

so our expression reduces to $\mathbf{R} \underline{\operatorname{Hom}}_{\operatorname{alg}^{*}(\mathbf{0} \sqcup \mathbf{0}) \downarrow s Q \mathrm{Dat}_{1}}\left(\operatorname{alg}^{*} \mathbf{1}, \mathcal{E}\right)$.

Now, in the simplicial category $\operatorname{alg}^{*}(\mathbf{0} \sqcup \mathbf{0}) \downarrow s Q D_{1} t_{1}$, a cofibrant replacement for $\operatorname{alg}^{*} \mathbf{1}$ is given by the object $\mathcal{C}$ with $\mathcal{C}(0,0)=\mathcal{C}(1,1)=\iota \bullet, \mathcal{C}(1,0)=\emptyset$ and $\mathcal{C}(0,1)^{\bullet}=$ $\Delta^{\bullet}$. The multiplicative structure is determined by setting $\omega_{0} * a=\partial^{0} a$ and $a * \omega_{1}=$ $\partial^{n+1} a$, for $a \in \Delta^{n}$ and $\omega_{0}, \omega_{1}$ the unique elements of $\mathcal{C}(0,0)^{1}$ and $\mathcal{C}(1,1)^{1}$.

Now,

$$
\begin{aligned}
& \underline{\operatorname{Hom}}_{\text {alg* } \left.^{*}(\mathbf{0} \sqcup \mathbf{0})\right\rfloor s Q \text { Dat }_{1}}(\mathcal{C}, \mathcal{E})= \\
& \left\{e \in \prod_{n}\left(\mathcal{E}(0,1)^{n}\right)^{\Delta^{n}}: \partial_{\mathcal{E}}^{i} e_{n}=\left(\partial_{\Delta}^{i}\right)^{*} e_{n+1}, \sigma_{\mathcal{E}}^{i} e_{n}=\left(\sigma_{\Delta}^{i}\right)^{*} e_{n-1}\right\}
\end{aligned}
$$

(which preserves (trivial) fibrations, proving that $\mathcal{C}$ is cofibrant).

This expression is just Tot $\mathcal{E}(0,1)$, where $\mathcal{E}(0,1)$ is given the cosimplicial structure of Lemma 5.8 , but $\mathcal{E}(0,1)=\mathcal{D}(\bar{x}, \bar{y})$, giving the required description.

\section{2. $\mathcal{N}$}

Definition 5.11. Given $\mathcal{D} \in s Q$ Dat, define $\mathcal{N}(\mathcal{D}) \in s \mathbb{S}$ by

$$
\mathcal{N}(\mathcal{D})_{n}:=\operatorname{Map}_{s Q \text { Dat }}^{h}\left(\operatorname{alg}^{*} \mathbf{n}, \mathcal{D}\right) .
$$

Proposition 5.12. Any Reedy fibrant replacement for $\mathcal{N}(\mathcal{D})$ is a Segal space.

Proof. If $W:=\mathcal{N}(\mathcal{D})$, we need to show that the maps

$$
W_{k} \rightarrow \overbrace{W_{1} \times_{\partial_{0}, W_{0} \partial_{1}}^{h} \cdots \times_{\partial_{0}, W_{0}, \partial_{1}}^{h} W_{1}}^{k}
$$

are weak equivalences for all $k$. 
Now, the right-hand side is given by

$$
\operatorname{Map}_{s Q D a t}^{h}\left(\operatorname{alg}^{*} \mathbf{1}_{\text {alg*0 }}^{\mathbf{L}} \cdots \cup_{\text {alg*0 }}^{\mathbf{L}} \operatorname{alg}^{*} \mathbf{1}, \mathcal{D}\right),
$$

so we need only show that $\operatorname{alg}^{*} \mathbf{1} \cup \underset{\text { alg*0 }}{\mathbf{L}} \cdots \cup_{\text {alg* }^{*}}^{\mathbf{L}} \operatorname{alg}^{*} \mathbf{1} \simeq \operatorname{alg}^{*} \mathbf{k}$.

A cofibrant replacement of the diagram for this homotopy colimit is given by taking $\Xi$ instead of $\operatorname{alg}^{*} \mathbf{0}$, and $P_{1}^{*} \operatorname{diag}^{*} \Xi$ instead of $\operatorname{alg}^{*} \mathbf{1}$. Thus the calculation of Proposition 5.7 can be interpreted as saying that

$$
\overbrace{\operatorname{alg}^{*} \cup_{\text {alg*0 }}^{\mathrm{L}} \cdots \cup_{\text {alg*0 }}^{\mathrm{L}} \operatorname{alg}^{*} \mathbf{1}}^{k} \simeq \operatorname{alg}^{*} \mathbf{k},
$$

which gives the required result.

Definition 5.13. Given a Segal space $W$, define Ob $W:=\left(W_{0}\right)_{0}$. For $x, y \in \mathrm{Ob} W$, define $\operatorname{map}_{W}(x, y):=\{x\} \times_{W_{0}, \partial_{1}} W_{1} \times_{\partial_{0}}\{y\} \in \mathbb{S}$. There is a natural category $\operatorname{Ho}(W)$ with objects $\mathrm{Ob} W$ and morphisms $\pi_{0} \operatorname{map}_{W}(x, y)$.

Definition 5.14. Recall from [Rez, 7.4] that a morphism $f: U \rightarrow V$ of Segal spaces is said to be a Dwyer-Kan equivalence if

1. $\mathrm{Ho}(f): \mathrm{Ho}(U) \rightarrow \mathrm{Ho}(V)$ is an equivalence of categories, and

2. for all $x, y \in \mathrm{Ob} U$, the $\operatorname{map} \operatorname{map}_{U}(x, y) \rightarrow \operatorname{map}_{V}(f x, f y)$ is a weak equivalence in $\mathbb{S}$.

Proposition 5.15. Given a fibrant object $\mathcal{D} \in s Q$ Dat, the natural transformation

$$
\mathcal{M C}(\mathcal{D}) \rightarrow \mathcal{N}(\mathcal{D})^{f}
$$

is a Dwyer-Kan equivalence of Segal spaces, where $(-)^{f}$ denotes Reedy fibrant replacement.

Proof. Corollary 5.3 amounts to saying that $\mathcal{M C}(\mathcal{D})$ is weakly equivalent to the homotopy fibre product of

$$
\mathcal{N}(\mathcal{D}) \rightarrow \operatorname{cosk}_{0} \operatorname{Map}_{s Q \text { Dat }}^{h}\left(\mathbf{0}, \mathcal{D}^{0}\right)^{\text {hor }} \leftarrow \operatorname{cosk}_{0}(\mathrm{Ob} \mathcal{D})^{\text {hor }} .
$$

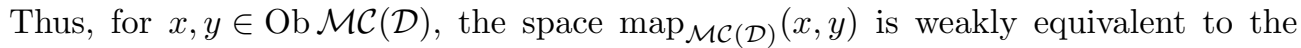
homotopy fibre product of

$$
\operatorname{map}_{\mathcal{N}(\mathcal{D})^{f}}(x, y) \rightarrow \operatorname{map}_{\operatorname{cosk}_{0} \operatorname{Map}_{s Q \mathrm{Dat}}^{h}\left(\mathbf{0}, \mathcal{D}^{0}\right)^{\text {hor }}}(x, y) \leftarrow \operatorname{map}_{\operatorname{cosk}_{0}(\mathrm{Ob} \mathcal{D})^{\text {hor }}}(x, y) .
$$

Now, for any $S \in \mathbb{S}$ and $x, y \in S_{0}, \operatorname{map}_{\operatorname{cosk}_{0}(S) \text { hor }}(x, y)=\{(x, y)\}$, so

$$
\operatorname{map}_{\mathcal{M C}(\mathcal{D})}(x, y) \simeq \operatorname{map}_{\mathcal{N}(\mathcal{D})^{f}}(x, y)
$$

It therefore remains only to show that the morphism $\operatorname{Ho}(\mathcal{M C}(\mathcal{D})) \rightarrow \operatorname{Ho}\left(\mathcal{N}(\mathcal{D})^{f}\right)$ of categories is essentially surjective. Since $\mathbf{0}$ is cofibrant, the map

$$
\mathrm{Ob} \mathcal{D} \rightarrow \operatorname{Map}_{s Q \text { Dat }}^{h}\left(\mathbf{0}, \mathcal{D}^{0}\right)
$$

is surjective on $\pi_{0}$. Any fibrant replacement $(\mathrm{Ob} \mathcal{D})^{f} \rightarrow \operatorname{Map}_{s Q \text { Dat }}^{h}\left(\mathbf{0}, \mathcal{D}^{0}\right)$ will also be surjective on $\pi_{0}$, and hence surjective on level 0 (by path-lifting). Therefore the map

$$
\mathrm{Ob} \mathcal{M C}(\mathcal{D}) \rightarrow \mathrm{Ob} \mathcal{N}(\mathcal{D})^{f} i
$$

is surjective on objects, so a fortiori essentially surjective. 
Definition 5.16. Given a Segal space $W$, let $W_{\text {hoequiv }} \subset W_{1}$ consist of components whose images in $\pi_{0} \operatorname{map}_{W}(x, y)$ are isomorphisms in $\mathrm{Ho}(W) . W$ is said to be a complete Segal space if the map $\sigma_{0}: W_{0} \rightarrow W_{\text {hoequiv }}$ is a weak equivalence.

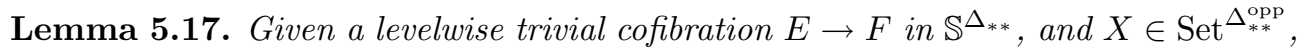
the map $X \underset{\leftarrow}{\times} \rightarrow X \underset{\leftarrow}{\leftarrow}$ is a trivial cofibration in $\mathbb{S}$, for $\underset{\leftarrow}{\leftarrow}$ as in Definition 1.2\%.

Proof. Since $\operatorname{Set}^{\Delta_{* *}}$ is equivalent to the category of augmented simplicial objects by Remark 1.2, a morphism $E \rightarrow F$ is a levelwise trivial cofibration precisely when $E^{0} \rightarrow$ $F^{0}$ is a trivial cofibration and $E^{\geqslant 1} \rightarrow F^{\geqslant 1}$ corresponds to a levelwise trivial cofibration of bisimplicial sets. By $[\mathbf{G J}]$ Theorem IV.3.9, this second condition is the same as being a Reedy trivial cofibration of bisimplicial sets.

Let $L^{n} E$ be the Reedy latching object of $E$ in $\operatorname{Set}^{\Delta_{* *}}$ (as in [Hov, Definition 5.2.2]). Explicitly, this is the quotient of $\coprod_{i=1}^{n-1} E^{n-1}$ by the relations $\left(\partial^{j} e\right)_{i} \sim\left(\partial^{i-1} e\right)_{j}$ for $e \in E^{n-2}$ and $i \leqslant j$. Note that $L^{0} E=L^{1} E=\emptyset$. For $E^{\geqslant 1} \rightarrow F^{\geqslant 1}$ to be a Reedy trivial cofibration says that the latching maps $E^{n} \cup_{L^{n} E} L^{n} F \rightarrow F^{n}$ are trivial cofibrations for all $n \geqslant 1$. Thus a morphism $E \rightarrow F$ is a levelwise trivial cofibration in $\mathbb{S}^{\Delta_{* *}}$ precisely when the latching maps are trivial cofibrations for all $n \geqslant 0$.

Let $(X \times E)^{(n)} \subset X \times E$ be the subspace generated by $X_{i} \times E^{i}$ for $i \leqslant n$, and let $N_{n} X=X_{n}-\left(\bigcup_{0 \leqslant r \leqslant n-1} \sigma_{r} X_{n-1}\right)$. Then

$$
(X \underset{\leftarrow}{\times} E)^{(n)}=(X \underset{\leftarrow}{\times})^{(n-1)} \cup_{\left(N_{n} X \times L^{n} E\right)}\left(N_{n} X \times E^{n}\right),
$$

so the map

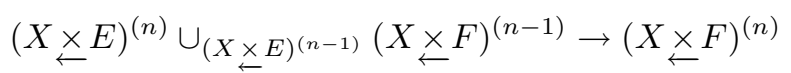

is a trivial cofibration.

We then proceed inductively to show that $(X \underset{\leftarrow}{\leftarrow} E)^{(n)} \rightarrow(X \underset{\leftarrow}{\leftarrow} F)^{(n)}$ is a trivial cofibration. The case $n=0$ is immediate, and by assuming the $n-1$ case we get a trivial cofibration

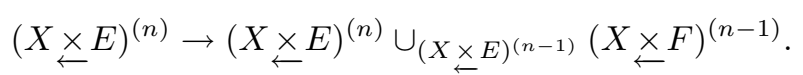

The result above then implies that $(X \underset{\leftarrow}{\leftarrow})^{(n)} \rightarrow(X \underset{\leftarrow}{\leftarrow} F)^{(n)}$ is a trivial cofibration, which completes the induction.

Thus $X \underset{\leftarrow}{\times} \rightarrow X \times F$ is a transfinite composition of trivial cofibrations, so must be a trivial cofibration, as required.

Proposition 5.18. If $\mathbb{I}$ is a small category and $\mathcal{D} \in s Q D a t$, then there are canonical equivalences

$$
\operatorname{Map}_{s \mathbb{S}}^{h}\left((B \mathbb{I})^{\text {hor }}, \mathcal{N}(\mathcal{D})\right) \simeq \operatorname{Map}_{s Q \operatorname{Dat}}^{h}\left(\operatorname{alg}^{*} \mathbb{I}, \mathcal{D}\right)
$$

Proof. Lemma 5.1 shows that $P_{n}^{*} \operatorname{diag}^{*} \Xi$ is a cofibrant replacement for alg* $\mathbf{n}$, so for $K \in \mathbb{S}$, 


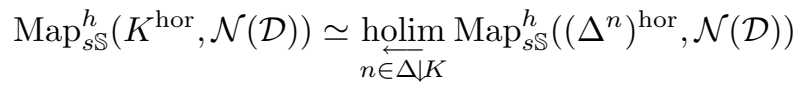

$$
\begin{aligned}
& \simeq \underset{n \in \operatorname{holim}_{n \in \Delta} K}{\operatorname{Map}_{s Q \operatorname{Dat}}^{h}}\left(P_{n}^{*} \operatorname{diag}^{*} \Xi, \mathcal{D}\right) \\
& \simeq \operatorname{Map}_{s Q \text { Dat }}^{h}\left(\underset{n \in(\overrightarrow{\Delta \mid K})^{\text {opp }}}{\operatorname{holim}} P_{n}^{*} \operatorname{diag}^{*} \Xi, \mathcal{D}\right) \\
& =\operatorname{Map}_{s Q \text { Dat }}^{h}\left(P_{K}^{*} \operatorname{diag}^{*} \Xi, \mathcal{D}\right) .
\end{aligned}
$$

Now, Lemma 1.35 gives a method for calculating the functor $P_{B \mathbb{I}}^{*}$. Since $\left(\operatorname{diag}^{*} \Xi\right)^{n, \bullet}$ $\in \mathbb{S}^{\Delta_{* *}}$ is levelwise contractible for all $n$ (by Proposition 4.33), a choice of point $x \in\left(\operatorname{diag}^{*} \Xi\right)^{n, 1}$ determines a levelwise trivial cofibration $\iota \bullet \rightarrow\left(\operatorname{diag}^{*} \Xi\right)^{n, \bullet}$ in $\mathbb{S}^{\Delta_{* *}}$. Therefore Lemma 5.17 implies that

$$
K(a, b) \underset{\longleftarrow}{\longleftarrow}(\iota \bullet) \rightarrow K(a, b) \underset{\longleftarrow}{\longleftarrow}\left(\operatorname{diag}^{*} \Xi\right)^{n, \bullet}
$$

is a trivial cofibration for all $a, b \in K_{0}$.

By Lemma 1.29, $\left(P_{B \mathbb{I}}^{*} \operatorname{diag}^{*} \Xi\right)(a, b)^{n}=(B \mathbb{I})(a, b) \times\left(\operatorname{diag}^{*} \Xi\right)^{n, \bullet}$, so the calculation above shows that the canonical map

$$
\left(P_{B \mathbb{I}}^{*} \operatorname{diag}^{*} \Xi\right)(a, b)^{n} \rightarrow(B \mathbb{I})(a, b) \underset{\longleftarrow}{\times}(\iota \bullet)
$$

is a weak equivalence; thus

$$
P_{B \mathbb{I}}^{*} \operatorname{diag}^{*} \Xi \rightarrow P_{B \mathbb{I}}^{*} \iota(\bullet, \bullet)
$$

is a weak equivalence, and $P_{B \mathbb{I}}^{*} \iota(\bullet, \bullet) \cong \operatorname{alg}^{*} \mathbb{I}$ as in Lemma 1.36.

Corollary 5.19. Any Reedy fibrant replacement of $\mathcal{N}(\mathcal{D})$ is a complete Segal space.

Proof. We know that $\mathcal{N}(\mathcal{D})^{f}$ is a Segal space by Proposition 5.12 . If we let $J$ be the simply connected groupoid on two objects, then [Rez, Proposition 6.4] implies that it suffices to show that the map

$$
\mathcal{N}(\mathcal{D})_{0}^{f} \rightarrow \operatorname{Map}_{s \mathbb{S}}^{h}\left((B J)^{\text {hor }}, \mathcal{N}(\mathcal{D})^{f}\right)
$$

is a weak equivalence.

By Proposition 5.18,

$$
\operatorname{Map}_{s \mathbb{S}}^{h}\left((B \mathbb{I})^{\text {hor }}, \mathcal{N}(\mathcal{D})\right) \simeq \operatorname{Map}_{s Q \text { Dat }}^{h}\left(\operatorname{alg}^{*} \mathbb{I}, \mathcal{D}\right),
$$

and alg* $J \simeq \operatorname{alg}^{*} \mathbf{0}$, since $J$ is simply connected. The result then follows by considering this equivalence in the cases $\mathbb{I}=J$ and $\mathbb{I}=\mathbf{0}$.

Definition 5.20. Given a simplicial category $\mathbb{I}$, let $B \mathbb{I} \in s \mathbb{S}$ be the nerve of $\mathbb{I}$, as considered in [Ber2, §8] (where it was denoted $R$ ). Explicitly, $(B \mathbb{I})_{n} \in \mathbb{S}$ is given by

$$
(B \mathbb{I})_{n}=\coprod_{x_{0}, \ldots, x_{n} \in \mathrm{Ob} \mathbb{I}} \underline{\operatorname{Hom}}_{\mathbb{I}}\left(x_{0}, x_{1}\right) \times \cdots \times \underline{\operatorname{Hom}}_{\mathbb{I}}\left(x_{n-1}, x_{n}\right) .
$$

In [Rez, Theorem 7.2], Rezk introduced the complete Segal space model structure $\mathcal{C S S}$ on the category $s \mathbb{S}$, whose fibrant objects are complete Segal spaces. In [Ber2], Bergner showed that there is a chain of Quillen equivalences between the model categories $\mathcal{C S S}$ and $s$ Cat. Therefore the following theorem can be interpreted as saying that $\mathcal{N}$ and $\mathcal{M C}$ are derived right adjoints to alg* $: s$ Cat $\rightarrow s Q$ Dat. 
Theorem 5.21. If $\mathbb{I}$ is a simplicial category and $\mathcal{D} \in s Q D$ Dat, then there are canonical equivalences

$$
\begin{aligned}
\operatorname{Map}_{s Q \mathrm{Dat}}^{h}\left(\operatorname{alg}^{*} \mathbb{I}, \mathcal{D}\right) & \simeq \operatorname{Map}_{s \mathbb{S}}^{h}(B \mathbb{I}, \mathcal{N}(\mathcal{D})) \\
& \simeq \operatorname{Map}_{\mathcal{C S \mathcal { S }}}^{h}(B \mathbb{I}, \mathcal{N}(\mathcal{D})) \\
& \simeq \operatorname{Map}_{\mathcal{C S \mathcal { S }}}^{h}\left(B \mathbb{I}, \mathcal{M C}\left(\mathcal{D}^{f}\right)\right),
\end{aligned}
$$

where $\mathcal{D}^{f}$ is a Reedy fibrant replacement of $\mathcal{D}$.

Proof. First note that we can regard $K \in s \mathbb{S}$ as a diagram $\Delta^{\text {opp }} \rightarrow \mathbb{S}$ and evaluate $K$ in the simplicial category $s \mathbb{S}$ as the coend $K=K \otimes_{\Delta^{\mathrm{opp}}}(\Delta)^{\mathrm{hor}}=\int^{n} K_{n}^{\mathrm{ver}} \times\left(\Delta^{n}\right)^{\mathrm{hor}}$ (in the notation of [Hir, Definition 18.3.2]). Since $K$ and $\Delta: \Delta^{\text {opp }} \rightarrow \mathbb{S}$ are both Reedy cofibrant, this means that $\operatorname{Map}_{s \mathbb{S}}^{h}(K, \mathcal{N}(\mathcal{D})$ ) is (assuming $\mathcal{N}(D)$ Reedy fibrant) the end $\int_{n} \operatorname{Map}_{s \mathbb{S}}^{h}\left(K_{n}^{\text {ver }} \times\left(\Delta^{n}\right)^{\text {hor }}, \mathcal{N}(\mathcal{D})\right)=\int_{n} \mathcal{N}(\mathcal{D})_{n}^{K_{n}}$. The proof of Proposition 5.18 now adapts to prove the first equivalence, replacing homotopy limits with ends.

For any $K \in s \mathbb{S}$, we may define $P_{K}^{*}: Q M M^{*}(\mathbb{S}) \rightarrow s Q \operatorname{Dat}(\mathbb{S})$, specialising to the functor $P_{K}^{*}$ of Lemma 1.35 when $K \in s$ Set. If $\mathbb{I}$ is a simplicial category, then for all $m$ the simplicial set $[n] \mapsto\left((B \mathbb{I})_{n}\right)_{m}$ is the nerve of the category $\mathbb{I}_{m}$, given by $\mathrm{Ob} \mathbb{I}_{m}=$ $\mathrm{ObI}$ and $\operatorname{Hom}_{\mathbb{I}_{m}}(x, y):=\underline{\operatorname{Hom}}_{\mathbb{I}}(x, y)_{i}$. Thus the description of $P_{B \mathbb{I}}^{*}$ in Lemma 1.35 generalises to simplicial categories $\mathbb{I}$, and, as in Proposition 5.18, the morphism

$$
P_{B \mathbb{I}}^{*} \operatorname{diag}^{*} \Xi \rightarrow P_{B \mathbb{I}}^{*} \iota(\bullet, \bullet)=\operatorname{alg}^{*} \mathbb{I}
$$

is a weak equivalence.

By Corollary $5.19, \mathcal{N}(\mathcal{D})^{f}$ is a complete Segal space, and since the identity functor $\mathcal{C S S} \rightarrow s \mathbb{S}$ is right Quillen for the Reedy model structure, the second equivalence follows. Proposition 5.15 gives the third equivalence, since [Rez, Theorem 7.7] shows that a morphism of Segal spaces becomes a weak equivalence in $\mathcal{C S S}$ if and only if it is a Dwyer-Kan equivalence.

\section{3. $\quad \mathcal{D E} \mathcal{L}$}

Definition 5.22. Let $\mathcal{D} \mapsto \mathcal{D}^{\mathbf{L G p d}}$ be the left-derived functor

$$
\mathrm{Ho}(s Q \mathrm{Dat}) \rightarrow \mathrm{Ho}(s Q \mathrm{Dpd})
$$

of the functor $\mathcal{D} \mapsto \mathcal{D}^{\text {Gpd }}$ from Definition 3.20.

Lemma 5.23. There is a natural equivalence $\left(\operatorname{alg}^{*} \mathbf{n}\right)^{\mathbf{L G p d}} \simeq \operatorname{alg}^{*}\left(\mathbf{n}^{\mathrm{Gpd}}\right)$.

Proof. By Lemma 1.36, $P_{n}^{*}$ diag* $\Xi$ is a cofibrant resolution of $\operatorname{alg}^{*} \mathbf{n}$, so we need to show that

$$
\left(P_{n}^{*} \operatorname{diag}^{*} \Xi\right)^{\mathrm{Gpd}} \simeq \operatorname{alg}^{*}\left(\mathbf{n}^{\mathrm{Gpd}}\right) .
$$

By Lemma 5.5, the natural morphism $f: \Xi \times \operatorname{alg}^{*} \mathbf{n} \rightarrow P_{n}^{*} \operatorname{diag}^{*} \Xi$ is a trivial cofibration in $s Q$ Dat $_{n}$ and hence also in $s Q$ Dat. Since $(-)^{\mathrm{Gpd}}: s Q$ Dat $\rightarrow s Q D$ pd is left Quillen, this implies that $f^{\text {Gpd }}$ is a trivial cofibration, so it suffices to show that $\left(\Xi \times \operatorname{alg}^{*} \mathbf{n}\right)^{\mathrm{Gpd}} \simeq \operatorname{alg}^{*}\left(\mathbf{n}^{\mathrm{Gpd}}\right)$. Now, $\left(\Xi \times \operatorname{alg}{ }^{*} \mathbf{n}\right)$ has objects $[0, n]$ and morphisms

$$
\left(\Xi \times \operatorname{alg}^{*} \mathbf{n}\right)^{\mathrm{Gpd}}(i, j)=\Xi
$$

for all $i, j$. Since $\Xi \simeq \iota \bullet$, this gives a weak equivalence $\left(\Xi \times \operatorname{alg}^{*} \mathbf{n}\right)^{\mathrm{Gpd}} \rightarrow \operatorname{alg}^{*}\left(\mathbf{n}^{\mathrm{Gpd}}\right)$, as required. 
Proposition 5.24. For $\mathcal{D} \in s Q D p d$ fibrant,

$$
\operatorname{Del}(\mathcal{D}) \simeq \operatorname{Map}_{s Q \operatorname{Dat}}^{h}\left(\operatorname{alg}^{*} \mathbf{n}, \mathcal{D}\right)
$$

for all $n \geqslant 0$.

Proof. Let Del $^{*}$ be the left adjoint to Del: $s Q D p d \rightarrow \mathbb{S}$, making it a left Quillen functor by Proposition 3.36. Since $\operatorname{Del}(\mathcal{D})_{0}=\coprod_{x \in \mathrm{Ob} \mathcal{D}} \operatorname{MC}(\mathcal{D}(x, x))$, it follows that $\operatorname{Del}^{*}\left(\Delta^{0}\right)=\Xi$, so $\operatorname{Del}^{*}\left(\Delta^{0}\right) \simeq \operatorname{alg}^{*} \mathbf{0}$. Since $\Delta^{\bullet}$ is a cofibrant cosimplicial resolution of $\Delta^{0}$ in $\mathbb{S}, \operatorname{Del}^{*}\left(\Delta^{\bullet}\right)$ is thus a cofibrant cosimplicial resolution of $\operatorname{alg}^{*} \mathbf{0}$, so

$$
\operatorname{Map}_{s Q D p d}^{h}\left(\operatorname{alg}^{*} \mathbf{0}, \mathcal{D}\right) \simeq\left([m] \mapsto \operatorname{Hom}_{s Q \operatorname{Dpd}}\left(\operatorname{Del}^{*}\left(\Delta^{m}\right), \mathcal{D}\right)\right)=\operatorname{Del}(\mathcal{D}),
$$

giving the case $n=0$.

For the general case, note that the inclusion functor $s Q D p d \rightarrow s Q D a t$ is right Quillen, with left adjoint given by groupoid completion, and so

$$
\operatorname{Map}_{s Q \operatorname{Dat}}^{h}\left(\operatorname{alg}^{*} \mathbf{n}, \mathcal{D}\right) \simeq \operatorname{Map}_{s Q \operatorname{Dpd}}^{h}\left(\left(\operatorname{alg}^{*} \mathbf{n}\right)^{\mathbf{L G p d}}, \mathcal{D}\right) \simeq \operatorname{Map}_{s Q \operatorname{Dpd}}^{h}\left(\operatorname{alg}^{*}\left(\mathbf{n}^{\mathrm{Gpd}}\right), \mathcal{D}\right),
$$

by Lemma 5.23. Since $\mathbf{n}^{\mathrm{Gpd}} \simeq \mathbf{0}$, this is just $\operatorname{Map}_{s Q D p d}^{h}\left(\operatorname{alg}^{*} \mathbf{0}, \mathcal{D}\right)$, which completes the proof.

Proposition 5.25. The functor $\mathcal{D E L}: s Q D p d \rightarrow s \mathbb{S}$ of Definition 3.38 is right Quillen for the complete Segal space model structure $\mathcal{C S S}$ of [Rez, Theorem 7.2]. In particular, for any $\mathcal{D} \in s Q D$ pd fibrant, the simplicial space $\mathcal{D E} \mathcal{L}(\mathcal{D})$ is a complete Segal space. In fact, $\mathcal{D E} \mathcal{L}(\mathcal{D})$ is then equivalent to the constant simplicial space $\operatorname{Del}(\mathcal{D})$.

Proof. By Corollary 3.39, we know that $\mathcal{D E} \mathcal{L}$ is right Quillen for the Reedy model structure. In order to prove the remaining statements, it suffices to show that for any fibration $f: \mathcal{D} \rightarrow \mathcal{E}$, the fibrations

$$
\left(f,\left(\partial_{i}\right)^{n}\right): \mathcal{D} \mathcal{E} \mathcal{L}(\mathcal{D})_{n} \rightarrow \mathcal{D E} \mathcal{L}(\mathcal{E})_{n} \times \operatorname{Del}(\mathcal{E}) \operatorname{Del}(\mathcal{D})
$$

are trivial fibrations for $i=0,1$ and for all $n$. This is equivalent to showing that the cofibrations

$$
\operatorname{Del}^{*}\left(\Delta^{m}\right) \cup_{\operatorname{Del}^{*}\left(\partial \Delta^{m}\right)}\left(\mathcal{D E} \mathcal{L}_{n}\right)^{*}\left(\partial \Delta^{m}\right) \rightarrow\left(\mathcal{D E} \mathcal{L}_{n}\right)^{*}\left(\Delta^{m}\right)
$$

are trivial cofibrations for all $m \geqslant 0$ (and both choices of map $\left(\partial_{i}\right)^{n *}: \operatorname{Del}^{*} \rightarrow \mathcal{D E} \mathcal{L}_{n}^{*}$ ), where $\left(\mathcal{D E} \mathcal{L}_{n}\right)^{*}: \mathbb{S} \rightarrow s Q$ Dpd is left adjoint to $\mathcal{D E} \mathcal{L}_{n}$.

Since $\mathcal{D E L}$ is right Quillen, the functor $\mathcal{D E} \mathcal{L}_{n}$ is right Quillen, so the proof of Proposition 5.24 adapts to show that the cosimplicial object $i \mapsto\left(\mathcal{D E} \mathcal{L}_{n}\right)^{*}\left(\Delta^{m}\right)$ is a cofibrant cosimplicial resolution of $\left(P_{n}^{*} \operatorname{diag}^{*} \Xi\right)^{\mathrm{Gpd}}$ in $s Q \mathrm{Dpd}$, which by Lemma 5.23 is equivalent to $\operatorname{alg}^{*}\left(\mathbf{n}^{\text {Gpd }}\right)$ and hence is equivalent to $\operatorname{alg}^{*} \mathbf{0}$. Therefore the cofibrations $\operatorname{Del}^{*}\left(\Delta^{m}\right) \rightarrow\left(\mathcal{D E} \mathcal{L}_{n}\right)^{*}\left(\Delta^{m}\right)$ are all weak equivalences. Moreover,

$$
\operatorname{Del}^{*}\left(\partial \Delta^{m}\right) \simeq \operatorname{holim}_{\Delta_{m-1,+}} \operatorname{alg}^{*} \mathbf{0}
$$

(for $\Delta_{m-1,+}$ the subcategory of $\Delta$ on objects $\leqslant m-1$ with only injective morphisms) 
and similarly for $\left(\mathcal{D E} \mathcal{L}_{n}\right)^{*}\left(\partial \Delta^{m}\right)$. Thus the cofibrations

$$
\operatorname{Del}^{*}\left(\partial \Delta^{m}\right) \rightarrow\left(\mathcal{D E} \mathcal{L}_{n}\right)^{*}\left(\partial \Delta^{m}\right)
$$

are also weak equivalences, giving a weak equivalence

$$
\operatorname{Del}^{*}\left(\Delta^{m}\right) \rightarrow \operatorname{Del}^{*}\left(\Delta^{m}\right) \cup_{\operatorname{Del}^{*}\left(\partial \Delta^{m}\right)}\left(\mathcal{D E} \mathcal{L}_{n}\right)^{*}\left(\partial \Delta^{m}\right) .
$$

Since $\operatorname{Del}^{*}\left(\Delta^{m}\right) \rightarrow\left(\mathcal{D E} \mathcal{L}_{n}\right)^{*}\left(\Delta^{m}\right)$ is a trivial cofibration (coming from the trivial cofibration $\left.\left(\partial^{i}\right)^{n}: \Delta^{0} \rightarrow \Delta^{n}\right)$, this gives the required result.

Corollary 5.26. For $\mathcal{D} \in s Q D p d$ fibrant, the morphism $\mathcal{M C}(\mathcal{D}) \rightarrow \mathcal{D E} \mathcal{L}(\mathcal{D})$ is a Dwyer-Kan equivalence, and $\mathcal{D} \mathcal{E} \mathcal{L}(\mathcal{D}) \simeq \mathcal{N}(\mathcal{D})$.

Proof. Proposition 5.24 implies that the constant simplicial space Del is a model for $\mathcal{N}$, so Proposition 5.25 shows that $\mathcal{D E L}$ must also be a model for $\mathcal{N}$. By Proposition 5.15, the morphism $\mathcal{M C}(\mathcal{D}) \rightarrow \mathcal{N}(\mathcal{D})^{f}$ is a Dwyer-Kan equivalence.

\section{Maurer-Cartan and classifying spaces}

In many cases, a simplicial quasi-descent datum $E$ has additional structure, and in this section we show how this simplifies the Segal spaces $\mathcal{M C}(E)$ and $\mathcal{D E} \mathcal{L}(E)$ (where appropriate). This will give descriptions which are not only simpler, but are related to more familiar formulae.

\subsection{Groups}

6.1.1. Cosimplicial simplicial groups

Definition 6.1. Let $c s$ Gp be the category of cosimplicial simplicial groups, equipped with its Reedy simplicial model structure over simplicial groups.

Example 6.2. Given a simplicial set $X$ and a simplicial group $G$, our main motivating example of a cosimplicial simplicial group is the complex $\mathrm{C}^{\bullet}(X, G)$ given by

$$
\mathrm{C}^{n}(X, G)_{m}:=G_{m}^{X_{n}},
$$

with cosimplicial operations $\partial^{i}:=G_{m}^{\partial_{i}}, \sigma^{i}:=G_{m}^{\sigma_{i}}$, and simplicial operations $\partial_{i}=\partial_{i}^{G}$, $\sigma_{i}=\sigma_{i}^{G}$.

Definition 6.3. Define MC: $c \mathrm{Gp} \rightarrow$ Set by

$$
\operatorname{MC}(G):=\mathrm{Z}^{1}(G)=\left\{\omega \in G^{1}: \sigma^{0} \omega=1 \partial^{1} \omega=\partial^{2} \omega \cdot \partial^{0} \omega\right\} .
$$

Definition 6.4. Define $\underline{\mathrm{MC}}: c s \mathrm{Gp} \rightarrow \mathbb{S}$ by $\underline{\mathrm{MC}}(G) \subset \prod_{n \geqslant 0}\left(G^{n+1}\right)^{\Delta^{n}}$, satisfying the conditions of [Pri3, Lemma 3.3]; i.e. the elements $\omega_{n} \in\left(G^{n+1}\right)^{\Delta^{n}}$ satisfy

$$
\begin{aligned}
\partial_{i} \omega_{n} & = \begin{cases}\partial^{i+1} \omega_{n-1} & i>0 \\
\left(\partial^{1} \omega_{n-1}\right) \cdot\left(\partial^{0} \omega_{n-1}\right)^{-1} & i=0,\end{cases} \\
\sigma_{i} \omega_{n} & =\sigma^{i+1} \omega_{n+1}, \\
\sigma^{0} \omega_{n} & =1 .
\end{aligned}
$$

Define $\mathrm{MC}: c s \mathrm{Gp} \rightarrow$ Set by $\mathrm{MC}(G)=\underline{\mathrm{MC}}(G)_{0}$, noting that this agrees with Definition 6.3 when $G \in c \mathrm{Gp}$. 
Remark 6.5. If $G \in c s \mathrm{Gp}$ is of the form $G=\mathrm{C}^{\bullet}(X, H)$, for $X \in \mathbb{S}$ and $H \in s \mathrm{Gp}$ as in Example 6.2, then [Pri3, Lemma 3.3] gives a canonical isomorphism $\mathrm{MC}(G) \cong$ $\operatorname{Hom}_{\mathbb{S}}(X, \bar{W} H)$, where $\bar{W}$ is the classifying space functor of [GJ, Ch V.4].

Definition 6.6. Define $\mathcal{E}: \operatorname{cs} \mathrm{Gp} \rightarrow Q M^{*}(\mathbb{S})$ by $\mathcal{E}(G)^{n}=G^{n}$, with identity $1 \in G^{0}$, operations $\partial_{\mathcal{E}(G)}^{i}=\partial_{G}^{i}, \sigma_{\mathcal{E}(G)}^{i}=\sigma_{G}^{i}$, and Alexander-Whitney product

$$
g * h=\left(\left(\partial_{G}^{m+1}\right)^{n} g\right) \cdot\left(\left(\partial_{G}^{0}\right)^{m} h\right)
$$

for $g \in G^{m}, h \in G^{n}$.

Observe that $\mathcal{E}$ is right Quillen and preserves weak equivalences. Denote its left adjoint by $\mathcal{E}^{*}$. Note that $\operatorname{MC}(G)=\operatorname{MC}(\mathcal{E}(G))$.

Note that the equivalence of Lemma 4.1 is just given by $\mathcal{E}: c \mathrm{Ab} \rightarrow Q M^{*}(\mathrm{Ab}, \times)$.

Proposition 6.7. For $G \in c s \mathrm{Gp}$ fibrant, there is a natural isomorphism

$$
\underline{\mathbf{R o m}}_{c s \mathrm{Gp}}\left(\mathcal{E}^{*}(\iota \bullet), G\right) \simeq \underline{\mathrm{MC}}(G)
$$

in $\operatorname{Ho}(\mathbb{S})$.

Proof. This is essentially the same as Proposition 3.11. First, note that $\mathcal{E}^{*}(\iota \bullet)^{n}$ is the free group on $n$ generators, with constant simplicial structure. If $s$ is the unique element of $(\iota \bullet)^{1}$, then the generators in level $n$ are given by $\partial^{i_{n-1}} \cdots \partial^{i_{2}} \partial^{i_{1}} s$, for $0<i_{1}<i_{2}<\cdots<i_{n-1} \leqslant n$. We then define $\Phi \in c s \mathrm{Gp}$ by

$$
\Phi^{n}:=\operatorname{Fr}\left(\coprod_{j<n} \Delta^{j}\right),
$$

where Fr denotes the free group functor. We give this the operations dual to those on $\bar{W} G$ in $\left[\mathbf{G J}\right.$, Ch. V]; i.e. for $x \in \Delta^{j} \subset \Phi^{n}$, we set

$$
\begin{gathered}
\partial_{\Phi}^{i}(x)= \begin{cases}\partial_{\Delta}^{i-n+j}(x) & i>n-j \\
\left(\partial_{\Delta}^{0} x\right) \cdot x & i=n-j \\
x & i<n-j,\end{cases} \\
\sigma_{\Phi}^{i}(x)= \begin{cases}\sigma_{\Delta}^{i-n+j}(x) & i \geqslant n-j \\
1 & i=n-j-1 \\
x & i<n-j-1 .\end{cases}
\end{gathered}
$$

Thus $\operatorname{Hom}_{s \mathrm{Gp}}\left(\Phi^{n}, G^{m}\right)=\left(\bar{W} G^{m}\right)_{n}$, with cosimplicial operations on $\Phi$ corresponding to simplicial operations on $\bar{W} G$, so we have

$$
\begin{aligned}
\operatorname{Hom}_{c s \mathrm{Gp}}(\Phi, G) & =\left\{f \in \prod_{n}\left(\bar{W} G^{n}\right)_{n}: \partial^{i} f_{n}=\partial_{i} f_{n+1} \sigma^{i} f_{n}=\sigma_{i} f_{n-1}\right\} \\
& =\operatorname{Hom}_{c \mathbb{S}}(\Delta, \bar{W} G)
\end{aligned}
$$

where $\Delta$ is the cosimplicial space given by $\Delta^{n}$ in level $n$.

The proof of [GJ, Lemma V.5.3] adapts to show that this is isomorphic to $\mathrm{MC}(G)$. Explicitly, $\underline{\omega} \in \mathrm{MC}(G)$ corresponds to the maps $\Delta^{n} \rightarrow \bar{W} G^{n}$ given by the element $\left(\omega_{n-1}, \partial^{0} \omega_{n-2}, \ldots,\left(\partial^{0}\right)^{n-1} \omega_{0}\right) \in\left(\bar{W} G^{n}\right)_{n}$. (This also implies that $\Phi$ is the group completion of the cosimplicial simplicial groupoid $G(\Delta)$, for $G$ the loop groupoid functor of $[\mathbf{G J}, \S \mathrm{V} .7]$.) Hence $\operatorname{Hom}_{c s \mathrm{Gp}}(\Phi, G) \cong \underline{\mathrm{MC}}(G)$. 
The inclusion $\mathrm{MC}\left(G_{0}\right) \hookrightarrow \underline{\mathrm{MC}}(G)$ corresponds to a map $\Phi \rightarrow \iota \bullet$. Since $\coprod_{i<n} \Delta^{i}$ is weakly equivalent to a disjoint union of $n-1$ points, this map must be a weak equivalence.

Now, we may show (similarly to Lemma 3.9, replacing $I^{n}$ by $\Delta^{n}$ ) that for all trivial fibrations $G \rightarrow H, \mathrm{MC}(G) \rightarrow \mathrm{MC}(H)$ is a surjection; hence $\Phi$ is cofibrant.

Thus, for $G$ fibrant,

$$
\mathbf{R} \underline{\operatorname{Hom}}\left(\mathcal{E}^{*}(\iota \bullet), G\right) \simeq \underline{\operatorname{Hom}}(\Phi, G) \simeq \underline{\operatorname{MC}}(G) .
$$

6.1.2. Equivalence of Maurer-Cartan spaces

Proposition 6.8. There are equivalences

$$
\underline{\operatorname{MC}}(\mathcal{E}(G)) \simeq \underline{\operatorname{MC}}(G)
$$

in $\mathbb{S}$, functorial in fibrant objects $G \in$ csGp. Here, the functors $\mathrm{MC}$ on the left and right are those from Definitions 3.5 and 6.4 respectively.

Proof. Since $G$ is fibrant, $\mathcal{E}(G)=\mathbf{R} \mathcal{E}(G)$. By Proposition 3.11,

$$
\underline{\mathrm{MC}}(\mathcal{E}(G)) \cong \mathbf{R H o m}_{Q M^{*}(\mathbb{S})}(\iota \bullet, \mathbf{R \mathcal { E }}(G)) \cong \underline{\operatorname{Hom}}_{c s \mathrm{Gp}}\left(\mathbf{L} \mathcal{E}^{*}(\iota \bullet), G\right) .
$$

Thus, by Proposition 6.7 , we need only show that $\mathbf{L} \mathcal{E}^{*}(\iota \bullet) \rightarrow \mathcal{E}^{*}(\iota \bullet)$ is a weak equivalence in $c s \mathrm{Gp}$. A model for $\mathbf{L} \mathcal{E}^{*}(\iota \bullet)$ is given by $\mathcal{E}^{*} \Xi$, for $\Xi$ from Definition 3.10 .

Since $\mathcal{E}^{*}$ is a left adjoint, it commutes with coequalisers, so $\pi_{0}\left(\mathcal{E}^{*} \Xi\right)^{n}=\mathcal{E}^{*}\left(\pi_{0} \Xi^{n}\right)=$ $\mathcal{E}^{*}\left((\iota \bullet)^{n}\right)$. Since $\mathcal{E}^{*}(\iota \bullet)^{n}$ is a discrete group, we need only show that the components of $\left(\mathcal{E}^{*} \Xi\right)^{n}$ are contractible for all $n$. This is equivalent to saying that the universal cover $U$ of $B \mathcal{E}^{*}(\iota \bullet)^{n}$ is contractible, which will follow from the Hurewicz theorem if $\mathrm{H}_{i}(U, \mathbb{Z})=0$ for all $i>1$. This is the same as saying that the homology groups $\mathrm{H}_{i}\left(\left(\mathcal{E}^{*} \Xi\right)^{n}, \mathbb{Z} \mathcal{E}^{*}(\iota \bullet)^{n}\right)$ are zero for all $i>1$.

Let $\operatorname{csRep}\left(\mathcal{E}^{*}(\iota \bullet)\right)$ be the category of abelian cosimplicial simplicial $\mathcal{E}^{*}(\iota \bullet)$-repbreak resentations and consider the functor $\operatorname{cs} \operatorname{Rep}\left(\mathcal{E}^{*}(\iota \bullet)\right) \rightarrow \operatorname{cs} \operatorname{Gp} \downarrow \mathcal{E}^{*}(\iota \bullet)$ given by $V \mapsto V \rtimes \mathcal{E}^{*}(\iota \bullet)$. This is a right adjoint, and it is right Quillen for the Reedy model structure on $c s \operatorname{Rep}\left(\mathcal{E}^{*}(\iota \bullet)\right)$. Denote the derived left adjoint by $\mathbf{L}$ cot and observe that for $V \in s \operatorname{Rep}\left(\mathcal{E}^{*}(\iota \bullet)^{n}\right)$,

$$
\begin{aligned}
\operatorname{Hom}_{\operatorname{Ho}\left(s \operatorname{Rep}\left(\mathcal{E}^{*}(\iota \bullet)^{n}\right)\right)}\left((\mathbf{L} \cot G)^{n}, V\right) & \cong \operatorname{Hom}_{\operatorname{Ho}\left(s \operatorname{Gp} \mid \mathcal{E}^{*}(\iota \bullet)^{n}\right)}\left(G^{n}, V[-i] \rtimes \mathcal{E}^{*}(\iota \bullet)^{n}\right) \\
& \cong \mathbb{H}^{1}\left(B\left(G^{n}\right), \bullet ; V\right),
\end{aligned}
$$

where the final group is hypercohomology (with coefficients in a complex of local systems). Thus

$$
\mathrm{H}_{i}(\mathbf{L} \cot G)^{n} \cong \mathrm{H}_{i+1}\left(B\left(G^{n}\right), \bullet ; \mathbb{Z} \mathcal{E}^{*}(\iota \bullet)^{n}\right) .
$$

It therefore suffices to show that $\mathrm{H}_{i}\left(\mathbf{L} \cot \mathcal{E}^{*} \Xi\right)=0$ for all $i \geqslant 1$.

Now, for $W \in \operatorname{cs} \operatorname{Rep}\left(\mathcal{E}^{*}(\iota \bullet)\right)$,

$$
\begin{aligned}
\underline{\operatorname{Hom}}_{c s \operatorname{Gp} \mathcal{E}^{*}(\iota \bullet)}\left(\mathcal{E}^{*} \Xi, W \rtimes \mathcal{E}^{*}(\iota \bullet)\right) & \cong \underline{\operatorname{Hom}}_{\left.Q M^{*}(\mathbb{S})\right) \mathcal{E} \mathcal{E}^{*}(\iota \bullet)}\left(\Xi, \mathcal{E}\left(W \rtimes \mathcal{E}^{*}(\iota \bullet)\right)\right) \\
& \cong \underline{\operatorname{Hom}}_{Q M^{*}(\mathbb{S})}\left(\Xi, \mathcal{E}\left(W \rtimes \mathcal{E}^{*}(\iota \bullet)\right) \times \mathcal{E \mathcal { E }}^{*}(\iota \bullet) \iota \bullet\right) \\
& \cong \underline{\operatorname{Hom}}_{Q M^{*}(\mathrm{Ab}, \times)}\left(\cot \Xi, \mathcal{E}\left(W \rtimes \mathcal{E}^{*}(\iota \bullet)\right) \times \mathcal{E}^{*}(\iota \bullet) \iota \bullet\right) .
\end{aligned}
$$

This leads us to consider the functor $\jmath: c s \operatorname{Rep}\left(\mathcal{E}^{*}(\iota \bullet)\right) \rightarrow c s \mathrm{Ab}$, given by

$$
W \mapsto \mathrm{C}\left(\mathcal{E}\left(W \rtimes \mathcal{E}^{*}(\iota \bullet)\right) \times_{\mathcal{E} \mathcal{E}^{*}(\iota \bullet)} \iota \bullet\right) .
$$


Explicitly, we see that this has objects $w \cdot \varpi_{m}$ in level $m$, for $\varpi_{m}$ the image of

$$
(\iota \bullet)^{m} \rightarrow \mathcal{E E}^{*}(\iota \bullet)^{m} .
$$

The operations are $\partial^{i}\left(w \cdot \varpi_{m}\right)=\partial^{i}(w) \cdot \varpi_{m+1}$ for $0<i \leqslant m, \sigma^{i}\left(w \cdot \varpi_{m}\right)=\sigma^{i}(w)$ $\cdot \varpi_{m-1}$,

$$
\begin{aligned}
\partial_{\jmath W}^{0}\left(w \cdot \varpi_{m}\right) & =\varpi_{1} *\left(w \cdot \varpi_{m}\right)=\left(\partial^{2}\right)^{m}\left(\varpi_{1}\right) \cdot \partial_{W}^{0}(w) \cdot \partial^{0}\left(\varpi_{m}\right) \\
& =\operatorname{ad}_{\left(\partial^{2}\right)^{m}\left(\varpi_{1}\right)}\left(\partial_{W}^{0} w\right) \cdot \varpi_{m+1}
\end{aligned}
$$

(as $\left.\varpi_{m+1}=\left(\partial^{2}\right)^{m}\left(\varpi_{1}\right) \cdot \partial^{0}\left(\varpi_{m}\right)\right)$, and

$$
\partial_{\jmath W}^{m+1}\left(w \cdot \varpi_{m}\right)=\left(w \cdot \varpi_{m}\right) * \varpi_{1}=\partial_{W}^{m+1} w \cdot \varpi_{m+1} .
$$

Thus $(\jmath W)^{n} \cong W^{n}$, with the same operations as $W$, except for

$$
\partial_{\jmath W}^{0}(w)=\left(\left(\partial^{2}\right)^{m} \varpi_{1}\right) \star \partial_{W}^{0}(w),
$$

where $\star$ denotes the group action of $\mathcal{E}^{*}(\iota \bullet)^{m+1}$ on $W^{m+1}$.

It therefore follows that the left adjoint $\jmath^{*}$ is given by $\left(\jmath^{*} U\right)^{n}=U^{n}\left[\mathcal{E}^{*}(\iota \bullet)^{n}\right]$, which is the free representation on generators $U^{n}$. This will have the same operations on generators as $U$, except that $\partial_{\jmath^{*} U}^{0}(u)=\left(\left(\partial^{2}\right)^{m} \varpi_{1}\right)^{-1} \star \partial_{U}^{0}(u)$.

For the natural Reedy model structure on $c s \operatorname{Rep}\left(\mathcal{E}^{*}(\iota \bullet)\right), \jmath^{*}$ is clearly left Quillen, so it follows that $\jmath$ is a right Quillen functor. Moreover, the descriptions above show that $\jmath$ and $\jmath^{*}$ both preserve weak equivalences. Thus

$$
\mathbf{L} \cot \mathcal{E}^{*} \Xi \simeq \jmath^{*} \mathbf{L} \cot (\iota \bullet),
$$

so $\mathrm{H}_{i}\left(\mathbf{L} \cot \mathcal{E}^{*} \Xi\right) \cong \mathrm{H}_{i} D_{c}(\mathbb{Z}[-1])$ by Lemma 4 .9. In particular, $\mathrm{H}_{i}\left(\mathbf{L} \cot \mathcal{E}^{*} \Xi\right)=0$ for all $i>0$, as required.

\subsubsection{Del}

Definition 6.9. For $G \in c s G p$, there is an adjoint action of $G^{0}$ on $\underline{\operatorname{MC}}(G)$, given by

$$
(g * \omega)_{n}=\left(\partial_{0}\left(\partial^{1}\right)^{n+1}\left(\sigma_{0}\right)^{n+1} g\right) \cdot \omega_{n} \cdot\left(\partial^{0}\left(\partial^{1}\right)^{n}\left(\sigma_{0}\right)^{n} g^{-1}\right),
$$

as in [Pri3, Definition 3.8].

We then define $\operatorname{Del}(G)$ to be the homotopy quotient $\operatorname{Del}(G)=\left[\underline{\operatorname{MC}}(G) /{ }^{h} G^{0}\right]:=$ $\underline{\operatorname{MC}}(G) \times G^{0} W G^{0} \in \mathbb{S}$.

Remark 6.10. If $G=\mathrm{C}^{\bullet}(X, H)$ for $X \in \mathbb{S}, H \in s \mathrm{Gp}$, then $\operatorname{Del}(G) \simeq \operatorname{Map}_{\mathbb{S}}(X, \bar{H})$. This is because

$$
\operatorname{Del}(G)_{n}=\operatorname{Hom}\left(X, \bar{W}\left(G^{\Delta^{n}}\right) \times\left(\operatorname{cosk}_{0} G_{0}\right)^{n}\right),
$$

and $[n] \mapsto \bar{W}\left(G^{\Delta^{n}}\right) \times\left(\operatorname{cosk}_{0} G_{0}\right)^{n}$ gives a fibrant simplicial resolution of $\bar{W} G$ in $\mathbb{S}$.

Proposition 6.11. The equivalence of Proposition 6.8 is $G^{0}$-equivariant, giving isomorphisms $\operatorname{Del}(\mathcal{E}(G)) \simeq \operatorname{Del}(G)$ in $\operatorname{Ho}(\mathbb{S})$, functorial in fibrant objects $G \in$ csGp.

Proof. If we let $\mathbb{Z} \in Q M^{*}(\mathbb{S})$ be the object given by $\mathbb{Z}$ in degree 0 and $\emptyset$ in higher degrees, then $E^{0} \cong \underline{\operatorname{Hom}}_{Q M^{*}(\mathbb{S})}(\mathbb{Z}, E)$, so the $E^{0}$-action on $\underline{\mathrm{MC}}(E)$ corresponds to a 
$\mathbb{Z}$-coaction on $\Xi$, i.e. a map

$$
\Xi \rightarrow \Xi \coprod \mathbb{Z}
$$

in $Q M^{*}(\mathbb{S})$, where $\coprod$ denotes coproduct in the category $Q M^{*}(\mathbb{S})$, satisfying a coassociativity condition.

Since $\mathcal{E}^{*}$ is a left adjoint, it preserves coproducts, so we get a coaction of $\mathcal{E}^{*}(\mathbb{Z})$ on $\mathcal{E}^{*}(\Xi)$, i.e. a coassociative map $\mathcal{E}^{*}(\Xi) \rightarrow \mathcal{E}^{*}(\Xi) \star \mathcal{E}^{*}(\mathbb{Z})$, where $\star$ denotes free product. Of course, $\underline{\operatorname{Hom}}_{c s \mathrm{Gp}}\left(\mathcal{E}^{*}(\mathbb{Z}), G\right) \cong \mathcal{E}(G)^{0}=G^{0}$.

Now, the morphisms

$$
\mathrm{MC}(\mathcal{E}(G)) \leftarrow \operatorname{MC}\left(\mathcal{E}(G)_{0}\right)=\operatorname{MC}\left(G_{0}\right) \rightarrow \operatorname{MC}(G)
$$

are equivariant with respect to the action of $G_{0}^{0}$. Since $\operatorname{Hom}_{c s G p}\left(\mathcal{E}^{*}(\mathbb{Z}), G\right) \cong G_{0}^{0}$, this amounts to saying that the weak equivalences

$$
\mathcal{E}^{*}(\Xi) \rightarrow \mathcal{E}^{*}(\iota \bullet) \leftarrow \Phi
$$

are $\mathcal{E}^{*}(\mathbb{Z})$-coequivariant, with the $\mathcal{E}^{*}(\mathbb{Z})$-coaction on $\Phi$ corresponding to the adjoint action of Definition 6.9.

We now make use of the fact that for $H \in s \mathrm{Gp}$ acting on $Y \in \mathbb{S}$, one model for the homotopy quotient $\left[Y /{ }^{h} H\right]$ is given by first forming the simplicial object $[Y / H]$ in the category of groupoids, then forming the nerve $B[Y / H]$ (which is a bisimplicial set), giving $\left[Y /{ }^{h} H\right] \simeq \operatorname{diag} B[Y / H]$.

Given $C \in c s \mathrm{Gp}$ equipped with a $\mathcal{E}^{*}(\mathbb{Z})$-coaction, we now define the cosimplicial diagram $\beta(C) \in(c s \mathrm{Gp})^{\Delta}$ by the property that

$$
\underline{\operatorname{Hom}}_{c s \mathrm{Gp}}(\beta(D), G)=B\left[\underline{\operatorname{Hom}}_{c s \mathrm{Gp}}(D, G) / G^{0}\right] \in(\mathbb{S})^{\Delta^{\mathrm{opp}}} .
$$

Explicitly, $\beta(D)^{n}=D \star \overbrace{\mathcal{E}^{*}(\mathbb{Z}) \star \mathcal{E}^{*}(\mathbb{Z}) \star \cdots \star \mathcal{E}^{*}(\mathbb{Z})}^{n}$.

Now $\mathcal{E}^{*}(\mathbb{Z})$ is just the cosimplicial group $\mathcal{E}^{*}(\mathbb{Z})^{n}=\overbrace{\mathbb{Z} \star \mathbb{Z} \star \cdots \star \mathbb{Z}}^{n+1}$, so $\mathcal{E}^{*}(\mathbb{Z})$ is levelwise cofibrant. Since $\mathcal{E}^{*}(\mathbb{Z}), \mathcal{E}^{*}(\Xi), \mathcal{E}^{*}(\iota \bullet)$ and $\Phi$ are all levelwise cofibrant, the morphisms $\beta\left(\mathcal{E}^{*}(\Xi)\right) \rightarrow \beta\left(\mathcal{E}^{*}(\iota \bullet)\right) \leftarrow \beta(\Phi)$ are (levelwise) weak equivalences.

In the Reedy model category $(c s \mathrm{Gp})^{\Delta}$, we now choose a cofibrant replacement $C$ for $\beta(\Phi)$, and a factorisation $\beta\left(\mathcal{E}^{*}(\Xi)\right) \rightarrow F \rightarrow \beta\left(\mathcal{E}^{*}(\iota \bullet)\right)$ with $\beta\left(\mathcal{E}^{*}(\Xi)\right) \rightarrow F$ a trivial cofibration and $F \rightarrow \beta\left(\mathcal{E}^{*}(\iota \bullet)\right)$ a trivial fibration. Since $C$ and $F$ are weakly equivalent in the Reedy model category $(\operatorname{cs} \mathrm{Gp})^{\Delta} \downarrow \beta\left(\mathcal{E}^{*}(\iota \bullet)\right)$, there is an explicit weak equivalence $f: C \rightarrow F$ in this category. Now, Reedy cofibrations are a fortiori levelwise cofibrations, so the objects $C^{n}, F^{n}$ are cofibrant in $c s \mathrm{Gp}$. We therefore have levelwise weak equivalences

$$
\underline{\operatorname{Hom}}_{c s \mathrm{Gp}}(\beta(\Phi), G) \rightarrow \underline{\operatorname{Hom}}_{c s \mathrm{Gp}}(C, G),
$$

and

$$
\underline{\operatorname{Hom}}_{c s \mathrm{Gp}}(F, G) \rightarrow \underline{\operatorname{Hom}}_{c s \mathrm{Gp}}\left(\beta\left(\mathcal{E}^{*}(\Xi)\right), G\right)
$$

in $(\mathbb{S})^{\Delta^{\text {opp }}}$, for all $G \in c s G$ p. Combining these with $f$ gives weak equivalences

$$
B\left[\underline{\mathrm{MC}}(G) / G^{0}\right] \rightarrow \underline{\operatorname{Hom}}_{c s \mathrm{Gp}}(C, G) \stackrel{f^{*}}{\longleftarrow} \underline{\operatorname{Hom}}_{c s \mathrm{Gp}}(F, G) \rightarrow B\left[\underline{\mathrm{MC}}(\mathcal{E}(G)) / G^{0}\right],
$$


in $(\mathbb{S})^{\Delta^{\mathrm{opp}}}$ and taking diagonals gives the required result that

$$
\operatorname{Def}(G) \simeq \operatorname{Def}(\mathcal{E}(G)) .
$$

\subsection{Lie algebras}

6.2.1. Nilpotent DGLAs

Definition 6.12. Let $D G_{\mathbb{Z}} \hat{N} L A$ denote the category of pro-nilpotent differential graded Lie algebras (DGLAs) over $k$.

Explicitly, a DGLA is a graded vector space $L=\bigoplus_{i \in \mathbb{Z}} L^{i}$ over $k$, equipped with operators [,] $L \times L \rightarrow L$ bilinear and $d: L \rightarrow L$ linear, satisfying:

1. $\left[L^{i}, L^{j}\right] \subset L^{i+j}$.

2. $[a, b]+(-1)^{\bar{a} \bar{b}}[b, a]=0$.

3. $(-1)^{\bar{c} \bar{a}}[a,[b, c]]+(-1)^{\bar{a} \bar{b}}[b,[c, a]]+(-1)^{\bar{b} \bar{c}}[c,[a, b]]=0$.

4. $d\left(L^{i}\right) \subset L^{i+1}$.

5. $d \circ d=0$.

6. $d[a, b]=[d a, b]+(-1)^{\bar{a}}[a, d b]$.

Here $\bar{a}$ denotes the degree of $a, \bmod 2$, for $a$ homogeneous.

A DGLA is said to be nilpotent if the lower central series $\Gamma_{n} L$ (given by $\Gamma_{1} L=L$, $\left.\Gamma_{n+1} L=\left[L, \Gamma_{n} L\right]\right)$ vanishes for $n \gg 0$.

Thus $D G_{\mathbb{Z}} \hat{N} L A$ is the category of pro-objects in the category of nilpotent DGLAs.

Definition 6.13. Given a pro-nilpotent Lie algebra $\mathfrak{g}$, define $\hat{\mathcal{U}}(\mathfrak{g})$ to be the prounipotent completion of the universal enveloping algebra of $\mathfrak{g}$, regarded as a pro-object in the category of algebras. As in [Qui1, Appendix A], this is a pro-Hopf algebra, and we define $\exp (\mathfrak{g}) \subset \hat{\mathcal{U}}(\mathfrak{g})$ to consist of elements $g$ with $\varepsilon(g)=1$ and $\Delta(g)=g \otimes g$, for $\varepsilon: \hat{\mathcal{U}}(\mathfrak{g}) \rightarrow k$ the augmentation (sending $\mathfrak{g}$ to 0 ), and $\Delta: \hat{\mathcal{U}}(\mathfrak{g}) \rightarrow \hat{\mathcal{U}}(\mathfrak{g}) \otimes \hat{\mathcal{U}}(\mathfrak{g})$ the comultiplication.

Since $k$ is assumed to have characteristic 0, exponentiation gives an isomorphism from $\mathfrak{g}$ to $\exp (\mathfrak{g})$, so we may regard $\exp (\mathfrak{g})$ as having the same elements as $\mathfrak{g}$, but with multiplication given by the Campbell-Baker-Hausdorff formula.

Definition 6.14. Given a $\mathbb{Z}$-graded pro-nilpotent DGLA $L^{\bullet}$, define the Maurer-Car$\tan$ set by

$$
\operatorname{MC}(L):=\left\{\omega \in L^{1} \mid d \omega+\frac{1}{2}[\omega, \omega]=0 \in L^{2}\right\} .
$$

Define the gauge group $\operatorname{Gg}(L)$ by $\operatorname{Gg}(L):=\exp \left(L^{0}\right)$, which acts on $\operatorname{MC}(L)$ by the gauge action $g(\omega)=g \cdot \omega \cdot g^{-1}-d g \cdot g^{-1}$, where $\cdot$ denotes multiplication in the universal enveloping algebra of $L$. That $g(\omega) \in \mathrm{MC}(L)$ is a standard calculation (see [Kon] or $[$ Man1]).

Let $\pi^{1}(L):=\operatorname{MC}(L) / \operatorname{Gg}(L)$ be the quotient set.

Lemma 6.15. If $e: L \rightarrow M$ has kernel $K$, with $[K, L]=0$, then there is an obstruction map $o_{e}: \pi^{1}(M) \rightarrow \mathrm{H}^{2}(K)$, with $o_{e}^{-1}(0)$ being the image of $\pi^{1}(L)$. Moreover, $\pi^{1}(L)$ is a principal $\mathrm{H}^{1}(K)$-bundle over $o_{e}^{-1}(0)$

Proof. This is well-known (see [Man1, §3], for instance). 
Definition 6.16. Let $O(\mathrm{MC})$ be the pro-nilpotent DGLA representing $\mathrm{MC}$, so

$$
\operatorname{Hom}(O(\mathrm{MC}), L) \cong \mathrm{MC}(L) \text {. }
$$

Explicitly, $O(\mathrm{MC})$ is the free pro-nilpotent graded Lie algebra on one generator $x$ in degree 1 , with differential determined by $d x=\frac{1}{2}[x, x]$.

Similarly, define $O(\mathrm{Gg})$ to represent $\mathrm{Gg}$; this is freely generated by $y$, $d y$, for $y \in O(\mathrm{Gg})^{0}$. Note that this has a cogroup structure in $D G_{\mathbb{Z}} \hat{N} L A$, coming from the group structure on $\mathrm{Gg}$.

Define $T$ and $O(T)$ by $T(L)=\operatorname{Hom}(O(T), L):=\exp \left(\mathrm{Z}^{0} L\right)$; this is freely generated by $z \in O(T)^{0}$, with $d z=0$. The embedding $T \hookrightarrow \mathrm{Gg}$ corresponds to the projection $y \mapsto z, d y \mapsto 0$, and $O(T)$ is a quotient cogroup of $O(\mathrm{Gg})$ in $D G_{\mathbb{Z}} \hat{N} L A$.

We may therefore regard $\mathrm{MC}, \mathrm{Gg}, T$ as being objects of the opposite category $\left(D G_{\mathbb{Z}} \hat{N} L A\right)^{\mathrm{opp}}$, which is a full subcategory of the category of presheaves on $D G_{\mathbb{Z}} \hat{N} L A$, via the Yoneda embedding.

Proposition 6.17. The map $q: \mathrm{Gg} \rightarrow \mathrm{MC}$ given by $g \mapsto g(0)$ gives an isomorphism between $\mathrm{MC}$ and the right quotient of $\mathrm{Gg}$ by $T$ in the category $\left(D G_{\mathbb{Z}} \hat{N} L A\right)^{\mathrm{opp}}$.

Proof. It is immediate that for $h \in T(L)$ we have $h(0)=0$, since $d h=0$. This gives a morphism $\operatorname{Gg}(L) / T(L) \rightarrow \mathrm{MC}(L)$, functorial in $L$, and we need to show that $\mathrm{MC}$ is the universal representable presheaf with this property, in other words that

$$
\mathrm{Gg} \times T \underset{\mu}{\stackrel{\mathrm{pr}_{1}}{\longrightarrow}} \mathrm{Gg} \stackrel{q}{\longrightarrow} \mathrm{MC}
$$

is a coequaliser in $\left(D G_{\mathbb{Z}} \hat{N} L A\right)^{\mathrm{opp}}$, where $\mu(g, t)=g \cdot t$.

The forgetful functor $U^{\text {opp }}$ from $D G_{\mathbb{Z}} \hat{N} L A$ to the category $G_{\mathbb{Z}} \hat{N} L A$ of pro-nilpotent $\mathbb{Z}$-graded Lie algebras preserves and reflects all equalisers, so $U:\left(D G_{\mathbb{Z}} \hat{N} L A\right)^{\text {opp }} \rightarrow$ $\left(G_{\mathbb{Z}} \hat{N} L A\right)^{\text {opp }}$ preserves and reflects all coequalisers. It therefore suffices to show that $U \mathrm{MC}=(U \mathrm{Gg}) /(U T)$.

Now, the forgetful functor $U^{\text {opp }}$ has a right adjoint $R$, given by $(R L)^{n}=L^{n} \times L^{n+1}$, with $\left[\left(a, a^{\prime}\right),\left(b, b^{\prime}\right)\right]=\left([a, b],\left[a^{\prime}, b\right]+(-1)^{\bar{a}}\left[a, b^{\prime}\right]\right)$ and $d\left(a, a^{\prime}\right)=\left(a^{\prime}, 0\right)$. This gives

$$
(U \mathrm{MC})(L) /(U \mathrm{Gg})(L)=\mathrm{MC}(R L) / \mathrm{Gg}(R L)=\pi^{1}(R L) .
$$

Applying $R$ to the tower $\cdots \rightarrow L /[L,[L, L]] \rightarrow L /[L, L]$ gives a tower of surjections satisfying the conditions of Lemma 6.15 . Since $\mathrm{H}^{*}(R M)=0$ for all $M$, this implies by induction that $\pi^{1}(R L)=0$ for all $L$. Thus $U$ Gg acts transitively on $U$ MC.

In particular, this means that the canonical element in $(U \mathrm{MC})\left(U^{\mathrm{opp}} O(\mathrm{MC})\right)$ is of the form $U q(s)$ for some $s \in(U \mathrm{Gg})\left(U^{\mathrm{opp}} O(\mathrm{MC})\right)$. Via the Yoneda embedding, $s$ is equivalent to the data of a section of $U q: U \mathrm{Gg} \rightarrow U \mathrm{MC}$. Since the fibres of $U q$ are precisely the $U T$-orbits, the map $U \mathrm{MC} \times U T \rightarrow U \mathrm{Gg}$ given by $(\omega, t) \mapsto s(\omega) \cdot t$ is an isomorphism. It follows immediately that the fork is a coequaliser, since

$$
(U \mathrm{MC} \times U T) / U T=U \mathrm{MC} .
$$


6.2.2. Cosimplicial simplicial groups

Definition 6.18. Given $G \in c s \mathrm{Gp}$, define the gauge group $\operatorname{Gg}(G)$ to be the subgroup of $\prod_{n} G_{n}^{n}$ consisting of those $\underline{g}$ satisfying

$$
\begin{array}{ll}
\partial_{i} g_{n}=\partial^{i} g_{n-1} & \forall i>0, \\
\sigma_{i} g_{n}=\sigma^{i} g_{n+1} \quad \forall i,
\end{array}
$$

similarly to [Pri3, Definition 3.5]. Note that $G_{0}^{0}$ can be regarded as a subgroup of $\operatorname{Gg}(G)$, setting $g_{n}=\left(\partial^{1}\right)^{n}\left(\sigma_{0}\right)^{n} g$, for $g \in G_{0}^{0}$. The group $T(G):=\operatorname{Tot}(G)_{0}$ is the subgroup of $\operatorname{Gg}(G)$ consisting of those $g$ for which $\partial_{0} g_{n}=\partial^{0} g_{n-1}$.

The action of Definition 6.9 extends to an action of $\operatorname{Gg}(G)$ on $\operatorname{MC}(G)$, given by $(g * \omega)_{n}=\left(\partial_{0} g_{n+1}\right) \cdot \omega_{n} \cdot\left(\partial^{0} g_{n}^{-1}\right)$, as in [Pri3, Definition 3.5].

Remark 6.19. If $G \in c s \mathrm{Gp}$ is of the form $G=\mathrm{C}^{\bullet}(X, H)$, for $X \in \mathbb{S}$ and $H \in s \mathrm{Gp}$, then [Pri3, Lemma 3.3 and Proposition 3.9] gives a canonical isomorphism $\operatorname{Gg}(G) \cong$ $\operatorname{Hom}_{\mathbb{S}}(X, W H)$ for $W H$ the canonical $H$-torsor on $\bar{W} H$, as in [GJ, Ch. V]. Moreover, $T(H) \cong \operatorname{Hom}_{\mathbb{S}}(X, H)$, and the map $q: \operatorname{Gg}(G) \rightarrow \mathrm{MC}(G)$ corresponding to the gauge action on 1 comes from the identification $\bar{W} G=G \backslash W G$ of [GJ, Ch. V].

Lemma 6.20. The functors $\mathrm{MC}, \mathrm{Gg}$ and $T$ are all representable in cs $\mathrm{Gp}$.

Proof. MC is represented by the object $\Phi$ defined in Proposition 6.7, which we will now denote by $O(\mathrm{MC})$, so $\operatorname{Hom}_{c s \mathrm{Gp}}(O(\mathrm{MC}), G) \cong \mathrm{MC}(G)$.

Define $O(T)$ by $O(T)^{n}=\operatorname{Fr}\left(\Delta^{n}\right)$ (where Fr denotes the free group functor), with cosimplicial operations coming from those on the cosimplicial space $\Delta^{\bullet}$; this gives $\operatorname{Hom}_{c s \mathrm{Gp}}(O(T), G) \cong T(G)$.

Similarly, define $O(\mathrm{Gg})$ to represent $\mathrm{Gg}$; this is given by $O(\mathrm{Gg})^{n}=\operatorname{Fr}\left(\coprod_{j \leqslant n} \Delta^{j}\right)$, with operations given on $x \in \Delta^{j} \subset O(\mathrm{Gg})^{n}$ by

$$
\begin{aligned}
& \partial_{O(\mathrm{Gg})}^{i}(x)= \begin{cases}\partial_{\Delta}^{i-n+j}(x) & i>n-j \\
x & i \leqslant n-j,\end{cases} \\
& \sigma_{O(\mathrm{Gg})}^{i}(x)= \begin{cases}\sigma_{\Delta}^{i-n+j}(x) & i>n-j \\
x & i \leqslant n-j .\end{cases}
\end{aligned}
$$

The isomorphism

$$
\operatorname{Gg}(G) \cong \operatorname{Hom}_{c s \mathrm{Gp}}(O(\mathrm{Gg}), G)
$$

is given by $g \in \operatorname{Gg}(G)$ mapping $\Delta^{j} \subset O(\mathrm{Gg})^{n}$ to $G^{n}$ via the element $\left(\partial_{0}\right)^{n-j} g_{n} \in G_{j}^{n}$.

Note that $O(\mathrm{Gg})$ has a cogroup structure in $c s \mathrm{Gp}$, coming from the group structure on Gg. Explicitly, this is the map $O(\mathrm{Gg}) \rightarrow O(\mathrm{Gg}) \star O(\mathrm{Gg})$ given by $x \mapsto x \star x$ for $x \in \Delta^{j} \subset O(\mathrm{Gg})^{n}$. The embedding $T \hookrightarrow \mathrm{Gg}$ corresponds to the quotient map $i: O(\mathrm{Gg}) \rightarrow O(T)$ given by mapping $\Delta^{j} \subset O(\mathrm{Gg})^{n}$ to $\Delta^{n} \subset O(T)^{n}$ via $\left(\partial_{\Delta}^{0}\right)^{n-j}$. Thus $O(T)$ is a quotient cogroup of $O(\mathrm{Gg})$ in $c s \mathrm{Gp}$.

We may therefore regard $\mathrm{MC}, \mathrm{Gg}$, and $T$ as being objects of the opposite category $(c s \mathrm{Gp})^{\text {opp }}$.

Proposition 6.21. The map $q: \mathrm{Gg} \rightarrow \mathrm{MC}$ given by $g \mapsto g * 1$ gives an isomorphism between $\mathrm{MC}$ and the right quotient of $\mathrm{Gg}$ by $T$ in the category $(c s \mathrm{Gp})^{\mathrm{opp}}$. 
Proof. Define $P \in c s G$ by

$$
P^{n}:=\operatorname{Fr}\left(\coprod_{j \leqslant n} \Delta^{j}\right)
$$

We give this the operations dual to those on $W G$ in $\left[\mathbf{G J}\right.$, Ch. V], i.e. for $x \in \Delta^{j} \subset P^{n}$, we set

$$
\begin{gathered}
\partial_{P}^{i}(x)= \begin{cases}\partial_{\Delta}^{i-n+j}(x) & i>n-j \\
\left(\partial_{\Delta}^{0} x\right) \cdot x & i=n-j \\
x & i<n-j\end{cases} \\
\sigma_{P}^{i}(x)= \begin{cases}\sigma_{\Delta}^{i-n+j}(x) & i \geqslant n-j \\
1 & i=n-j-1 \\
x & i<n-j-1 .\end{cases}
\end{gathered}
$$

In particular, $O(\mathrm{MC})^{n}$ is the simplicial subgroup of $P^{n}$ on generators $\coprod_{j<n} \Delta^{j}$, making $O(\mathrm{MC})$ a subobject of $P$.

Now, [Pri3, Proposition 3.9] adapts to give an isomorphism $\psi: P \rightarrow O(\mathrm{Gg})$, given by mapping $x \in \Delta^{j} \subset P^{n}$ to $\left(\partial_{\Delta}^{0} x\right) \cdot x^{-1}$ when $j<n$, and $x^{-1}$ when $j=n$.

The right action of $T$ on $\mathrm{Gg}$ corresponds to the coaction $\mu: O(\mathrm{Gg}) \rightarrow O(\mathrm{Gg}) \star O(T)$ given by mapping $x \in \Delta^{j} \subset O(\mathrm{Gg})^{n}$ to $x_{\mathrm{Gg}} \cdot\left(\left(\partial_{\Delta}^{0}\right)^{n-j} x\right)_{T}$, where $y_{\mathrm{Gg}}$ and $y_{T}$ denote the copies of $y$ in $O(\mathrm{Gg})^{n}$ and in $O(T)^{n}$, respectively. There is an obvious isomorphism $P^{n} \cong O(\mathrm{MC})^{n} \star O(T)^{n}$, since $O(T)^{n}=\operatorname{Fr}\left(\Delta^{n}\right)$, and this is equivariant for the $O(T)$ coaction (with trivial coaction on $O(\mathrm{MC})$ ), since $x \in \Delta^{j} \subset P^{n}$ has $\mu(\psi(x))=\psi(x)$ $\in O(\mathrm{Gg})$ for $j<n$, and $\mu(\psi(x))=\psi(x) \cdot i(x)$ when $j=n$.

Therefore

$$
\mathrm{Gg} \times T \underset{\mu}{\stackrel{\mathrm{pr}_{1}}{\longrightarrow}} \mathrm{Gg} \stackrel{q}{\longrightarrow} \mathrm{MC}
$$

is a coequaliser in $c s \mathrm{Gp}^{\text {opp }}$, as required.

6.2.3. Lie algebras to groups

Definition 6.22. Given an $\mathbb{N}_{0}$-graded DGLA $L$, let $D L$ be its cosimplicial denormalisation. Explicitly,

$$
D^{n} L:=\bigoplus_{\substack{m+s=n \\ 1 \leqslant j_{1}<\cdots<j_{s} \leqslant n}} \partial^{j_{s}} \cdots \partial^{j_{1}} L^{m}
$$

We then define operations $\partial^{j}$ and $\sigma^{i}$ using the cosimplicial identities, subject to the conditions that $\sigma^{i} L=0$ and $\partial^{0} v=d v-\sum_{i=1}^{n+1}(-1)^{i} \partial^{i} v$ for all $v \in L^{n}$.

We now have to define the Lie bracket $\llbracket$, from $D^{n} L \otimes D^{n} L$ to $D^{n} L$. Given a finite set $I$ of distinct strictly positive integers, write $\partial^{I}=\partial^{i_{s}} \cdots \partial^{i_{1}}$, for $I=\left\{i_{1}, \ldots, i_{s}\right\}$, with $i_{1}<\cdots<i_{s}$. The Lie bracket is then defined on the basis by

$$
\llbracket \partial^{I} v, \partial^{J} w \rrbracket:= \begin{cases}\partial^{I \cap J}(-1)^{(J \backslash I, I \backslash J)}[v, w] & v \in L^{|J \backslash I|}, w \in L^{|I \backslash J|}, \\ 0 & \text { otherwise, }\end{cases}
$$

where for disjoint sets $S, T$ of integers, $(-1)^{(S, T)}$ is the sign of the shuffle permutation of $S \sqcup T$ which sends the first $|S|$ elements to $S$ (in order), and the remaining $|T|$ elements 
to $T$ (in order). Beware that this formula cannot be used to calculate $\llbracket \partial^{I} v, \partial^{J} w \rrbracket$ when $0 \in I \cup J$ (for the obvious generalisation of $\partial^{I}$ to finite sets $I$ of distinct non-negative integers).

Theorem 6.23. Given a simplicial pro-nilpotent $\mathbb{N}_{0}$-graded DGLA $L:$, there are canonical isomorphisms

$$
\operatorname{Gg}(\exp (D L)) \cong \operatorname{Gg}\left(\operatorname{Tot}^{\Pi} N^{s} L\right), \quad \mathrm{MC}(\exp (D L)) \cong \operatorname{MC}\left(\operatorname{Tot}^{\Pi} N^{s} L\right),
$$

which are compatible with the respective gauge actions. Here, $D$ is cosimplicial denormalisation and $N^{s}$ is simplicial normalisation (as in Definition 4.6). This isomorphism acts as the identity on the subgroups

$$
\exp \left(L_{0}^{0}\right) \leqslant \operatorname{Gg}(\exp (D L)) \quad \text { and } \quad \exp \left(L_{0}^{0}\right) \leqslant \mathrm{Gg}\left(\operatorname{Tot}^{\Pi} N^{s} L\right)
$$

Proof. In order to keep track of the various gradings in these categories, we will write $D G_{\mathbb{Z}}$ for $\mathbb{Z}$-graded cochain complexes, $D G$ for cochain complexes in non-negative degrees, and $d g$ for chain complexes in non-negative degrees.

On the category $s D G \hat{N} L A$ of simplicial pro-nilpotent $\mathbb{N}_{0}$-graded DGLA, these functors are all representable, since $\exp , D$, $\operatorname{Tot}^{\Pi}$ and $N^{s}$ are all right adjoints, so

$$
\begin{aligned}
& \operatorname{Hom}_{s D G \hat{N} L A}\left(D^{*} \exp ^{*} O(\mathrm{Gg}), L\right) \cong \mathrm{Gg}(\exp (D L)), \\
& \operatorname{Hom}_{s D G \hat{N} L A}\left(D^{*} \exp ^{*} O(\mathrm{MC}), L\right) \cong \mathrm{MC}(\exp (D L)), \\
& \operatorname{Hom}_{s D G \hat{N} L A}\left(\left(N^{s}\right)^{*}\left(\operatorname{Tot}^{\Pi}\right)^{*} O(\mathrm{Gg}), L\right) \cong \mathrm{Gg}\left(\operatorname{Tot}^{\Pi} N^{s} L\right), \\
& \operatorname{Hom}_{s D G \hat{N} L A}\left(\left(N^{s}\right)^{*}\left(\operatorname{Tot}^{\Pi}\right)^{*} O(\mathrm{MC}), L\right) \cong \operatorname{MC}\left(\operatorname{Tot}^{\Pi} N^{s} L\right),
\end{aligned}
$$

for $\exp ^{*}: c s \mathrm{Gp} \rightarrow \operatorname{cs} \hat{N} L A$,

$$
D^{*}: c s \hat{N} L A \rightarrow s D G \hat{N} L A,\left(\operatorname{Tot}^{\Pi}\right)^{*}: D G_{\mathbb{Z}} \hat{N} L A \rightarrow d g D G \hat{N} L A
$$

and $\left(N^{s}\right)^{*}: d g D G \hat{N} L A \rightarrow s D G \hat{N} L A$ the corresponding left adjoints.

We may therefore regard the functors $D^{*} \exp ^{*} \mathrm{Gg}, D^{*} \exp ^{*} \mathrm{MC},\left(N^{s}\right)^{*}\left(\operatorname{Tot}^{\Pi}\right)^{*} \mathrm{Gg}$ and $\left(N^{s}\right)^{*}\left(\operatorname{Tot}^{\Pi}\right)^{*} \mathrm{MC}$ as objects of $(s D G \hat{N} L A)^{\text {opp }}$ and likewise for $D^{*} \exp ^{*} T$ and $\left(N^{s}\right)^{*}\left(\operatorname{Tot}^{\Pi}\right)^{*} T$.

Now, the isomorphism $\operatorname{Gg}(\exp (D L)) \cong \operatorname{Gg}\left(\operatorname{Tot}^{\Pi} N^{s} L\right)$ simply follows from the proof of the Dold-Kan correspondence, which generalises to give an equivalence of categories between $\mathbb{N}_{0}$-graded complexes and "simplicial abelian groups without $\partial_{0}$ ". This isomorphism maps the subgroup $T(\exp (D L))$ to $T\left(\operatorname{Tot}^{\Pi} N^{s} L\right)$ isomorphically, by the usual Dold-Kan correspondence.

The isomorphism $D^{*} \exp ^{*} \mathrm{MC} \cong\left(N^{s}\right)^{*}\left(\operatorname{Tot}^{\Pi}\right)^{*} \mathrm{MC}$ follows by taking the right quotients

$$
\left(D^{*} \exp ^{*} \mathrm{Gg}\right) /\left(D^{*} \exp ^{*} T\right) \cong\left(\left(N^{s}\right)^{*}\left(\operatorname{Tot}^{\Pi}\right)^{*} \mathrm{Gg}\right) /\left(\left(N^{s}\right)^{*}\left(\operatorname{Tot}^{\Pi}\right)^{*} T\right)
$$

in $(s D G \hat{N} L A)^{\mathrm{opp}}$, by Propositions 6.21 and 6.17 .

Remark 6.24. Note that this gives a shorter and more conceptual proof of [Pri3, Theorems 4.39 and 4.44], and in that context we may shorten the arguments, replacing Proposition 6.21 with [Pri3, Proposition 3.9]. 
Definition 6.25. Define $\operatorname{MCTot}^{\Pi} N^{s}: s D G \hat{N} L A \rightarrow \mathbb{S}$ by

$$
\underline{\operatorname{MCTot}}{ }^{\Pi} N^{s}(L)_{n}:=\operatorname{MC}\left(\operatorname{Tot}^{\Pi} N^{s}\left(L^{\Delta^{n}}\right)\right)
$$

and define Del: $s D G \hat{N} L A \rightarrow \mathbb{S}$ to be the homotopy quotient

$$
\text { Del }:=\left[\underline{\mathrm{MC} \mathrm{Tot}^{\Pi} N^{s}} /^{h} \exp \left(L^{0}\right)\right] ;
$$

i.e.

$$
\operatorname{Del}(L):=\underline{\operatorname{MCTot}}{ }^{\Pi} N^{s}(L) \times^{\exp \left(L^{0}\right)} W \exp \left(L^{0}\right),
$$

where $\exp \left(L^{0}\right)_{n} \subset \operatorname{Gg}\left(L^{\Delta^{n}}\right)$ acts via the gauge action.

Remark 6.26. This is essentially the functor used to construct derived formal stacks in $[$ Hin].

Corollary 6.27. There are canonical isomorphisms

$$
\operatorname{Del}(L) \cong \operatorname{Del}(\exp (D L))
$$

in $\mathbb{S}$, functorial in $L \in s D G \hat{N} L A$.

Proof. By Theorem 6.23, $\underline{\mathrm{MC} \mathrm{Tot}^{\Pi} N^{s}}(L) \cong \underline{\mathrm{MC}}(L)$. This isomorphism is equivariant with respect to the action of the simplicial group

$$
\exp \left(L^{0}\right)_{n}=\exp \left(\left(L^{\Delta^{n}}\right)_{0}^{0} \leqslant \operatorname{Gg}\left(L^{\Delta^{n}}\right),\right.
$$

so it gives the required isomorphism on taking homotopy quotients.

\section{Appendix A. Quasi-monads, quasi-comonads and quasi-distributivity}

To date, the main context in which s.h. T-algebras have been studied is when the monad $T$ comes from an operad. This is partly because these were the only cases for which a satisfactory theory of morphisms was developed in $[\mathbf{C L M}]$. Since this difficulty was resolved for general monads (and even distributive monad-comonad pairs) in Section 3.5, we now look into how related constructions adapt to this generality.

In $[\mathbf{K S}]$, free resolutions of operads were exploited to study deformations of algebras. Since an objectwise weak equivalence $T^{\prime} \rightarrow T$ of arbitrary monads on a simplicial category gives a weak equivalence of the associated simplicial quasi-descent data (from Proposition 2.9), the respective Segal spaces of s.h. T-algebras are weakly equivalent. This means that many of the ideas from $[\mathbf{K S}]$ carry over to arbitrary monads.

However, in some settings, such as $[\mathbf{v d L}]$, operads are too restrictive, and homotopy operads have to be used instead. We now introduce quasi-monads and quasi-comonads, which give a context sufficiently general to be analogous to homotopy operads, while providing a natural generalisation of the constructions of Section 2.

\section{A.1. Quasi-monads and quasi-comonads}

Definition A.1. Define a quasi-monad (resp. a quasi-comonad) on a category $\mathcal{B}$ to be a quasi-monoid (resp. quasi-comonoid), as in Definition 1.4, in the monoidal category $(\operatorname{End}(\mathcal{B}), \circ)$ of endofunctors of $\mathcal{B}$. 
Substituting the monoidal category $(\operatorname{End}(\mathcal{B})$, o $)$ into Lemma 1.5 yields the following two lemmas.

Lemma A.2. A quasi-monad consists of functors $\top_{n}: \mathcal{B} \rightarrow \mathcal{B}$, together with the following data:

$$
\begin{aligned}
& \mu_{i}: \top_{n+1} \Longrightarrow \top_{n} \quad 1 \leqslant i \leqslant n, \\
& \eta_{i}: \top_{n-1} \Longrightarrow \top_{n} \quad 0 \leqslant i<n,
\end{aligned}
$$

an associative coproduct $\xi_{m n}: \top_{m+n} \Longrightarrow \top_{m} \circ \top_{n}$, with coidentity $\xi_{0}: \top_{0} \Longrightarrow \mathrm{id}$, satisfying:

1. $\mu_{i} \mu_{j}=\mu_{j-1} \mu_{i} \quad i<j$.

2. $\eta_{i} \eta_{j}=\eta_{j+1} \eta_{i} \quad i \leqslant j$.

3. $\mu_{i} \eta_{j}= \begin{cases}\eta_{j-1} \mu_{i} & i<j \\ \mathrm{id} & i=j, i=j+1 \\ \eta_{j} \mu_{i-1} & i>j+1\end{cases}$

4. $\left(\mu_{i} \top_{n}\right) \xi_{m+1, n}=\xi_{m n} \mu_{i}$.

5. $\left(\top_{m} \mu_{i}\right) \xi_{m, n+1}=\xi_{m n} \mu_{i+m}$.

6. $\left(\eta_{i} \top_{n}\right) \xi_{m-1, n}=\xi_{m n} \eta_{i}$.

7. $\left(\top_{m} \eta_{i}\right) \xi_{m, n-1}=\xi_{m n} \eta_{i+m}$.

Lemma A.3. A quasi-comonad consists of functors $\perp^{n}: \mathcal{B} \rightarrow \mathcal{B}$, together with the following data:

$$
\begin{gathered}
\Delta^{i}: \perp^{n} \Longrightarrow \perp^{n+1} \quad 1 \leqslant i \leqslant n \\
\varepsilon^{i}: \perp^{n} \Longrightarrow \perp^{n-1} \quad 0 \leqslant i<n,
\end{gathered}
$$

an associative product $\zeta^{m n}: \perp^{m} \circ \perp^{n} \Longrightarrow \perp^{m+n}$, with identity $\zeta^{0}$ : id $\Longrightarrow \perp^{0}$, satisfying:

1. $\Delta^{j} \Delta^{i}=\Delta^{i} \Delta^{j-1} \quad i<j$.

2. $\varepsilon^{j} \varepsilon^{i}=\varepsilon^{i} \varepsilon^{j+1} \quad i \leqslant j$.

3. $\varepsilon^{j} \Delta^{i}= \begin{cases}\Delta^{i} \varepsilon^{j-1} & i<j \\ \mathrm{id} & i=j, i=j+1 \\ \Delta^{i-1} \varepsilon^{j} & i>j+1\end{cases}$

4. $\zeta^{m+1, n}\left(\Delta^{i} \perp^{n}\right)=\Delta^{i} \zeta^{m n}$.

5. $\zeta^{m, n+1}\left(\perp^{m} \Delta^{i}\right)=\Delta^{i+m} \zeta^{m n}$.

6. $\zeta^{m-1, n}\left(\varepsilon^{i} \perp^{n}\right)=\varepsilon^{i} \zeta^{m n}$.

7. $\zeta^{m, n-1}\left(\perp^{m} \varepsilon^{i}\right)=\varepsilon^{i+m} \zeta^{m n}$.

Lemma A.4. A quasi-monad on $\mathcal{B}$ gives rise to a quasi-descent datum enriching $\mathcal{B}$, given by

$$
\mathcal{H o m}\left(B, B^{\prime}\right)^{n}:=\operatorname{Hom}_{\mathcal{B}}\left(\top_{n} B, B^{\prime}\right) \text {. }
$$

In particular, this allows us to define Maurer-Cartan sets. We also have a notion of homotopy monad on a simplicial category (when the $\xi$ are all weak equivalences). Dually, we have the same constructions for quasi-comonads. 


\section{A.2. Distributivity for quasi-monads and quasi-comonads}

We now need to describe a distributivity transformation $\lambda$ for a quasi-monad $T$ and a quasi-comonad $\perp$. We wish to enrich $\mathcal{B}$ to a quasi-descent datum by setting

$$
\mathcal{H o m}_{\mathcal{B}}\left(B, B^{\prime}\right)^{n}:=\operatorname{Hom}\left(\top_{n} B, \perp^{n} B^{\prime}\right),
$$

so we need natural transformations

$$
\lambda_{m}^{n}: \top_{m} \perp^{n} \Longrightarrow \perp^{n} \top_{m} .
$$

with the following diagrams commuting:

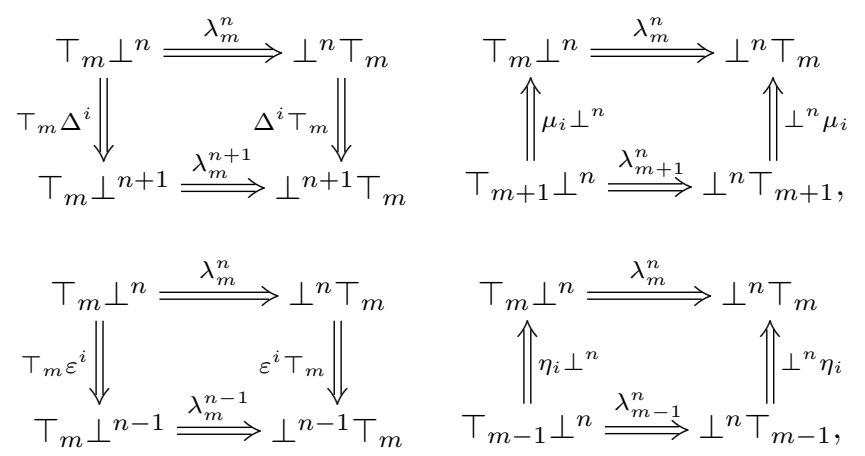

together with unit rules

$$
\left(\xi_{0} \perp^{n}\right)=\left(\perp^{n} \xi_{0}\right) \circ \lambda_{0}^{n} \quad \zeta^{0} \top_{m}=\lambda_{m}^{0} \circ\left(\top_{m} \zeta^{0}\right)
$$

and associativity rules

$$
\begin{gathered}
\left(\lambda_{p}^{n} \top_{q}\right) \circ\left(\top_{p} \lambda_{q}^{n}\right) \circ\left(\xi_{p q} \perp^{n}\right)=\left(\perp^{n} \xi_{p q}\right) \circ \lambda_{p+q}^{n}, \\
\left(\perp^{p} \lambda_{m}^{q}\right) \circ\left(\lambda_{m}^{p} \perp^{q}\right) \circ\left(\top_{m} \zeta^{p q}\right)=\left(\zeta^{p q} \top_{m}\right) \circ \lambda_{m}^{p+q} .
\end{gathered}
$$

Lemma A.5. Given a category $\mathcal{B}$, a quasi-monad $\top$ and a quasi-comonad $\perp$ on $\mathcal{B}$, together with a quasi-distributivity transformation $\lambda$ satisfying the conditions above, there is a quasi-descent datum on $\mathcal{B}$ given by setting

$$
\operatorname{Hom}_{\mathcal{B}}\left(B, B^{\prime}\right)^{n}:=\operatorname{Hom}\left(\top_{n} B, \perp^{n} B^{\prime}\right) .
$$

Proof. We define the structures on $\mathcal{H o m}_{\mathcal{B}}$ by

$$
\begin{aligned}
\partial^{i} x & =\Delta^{i} \circ x \circ \mu_{i}, \\
\sigma^{i} x & =\varepsilon^{i} \circ x \circ \eta_{i}, \\
x * y & =\zeta^{n m} \circ\left(\perp^{n} x\right) \circ \lambda_{m}^{n} \circ\left(\top_{m} y\right) \circ \xi_{m n},
\end{aligned}
$$

for $x \in \mathcal{H o m}_{\mathcal{B}}\left(B^{\prime}, B^{\prime \prime}\right)^{m}$ and $y \in \mathcal{H o m}_{\mathcal{B}}\left(B, B^{\prime}\right)^{n}$, with identity $\zeta^{0} \circ \xi_{0}$.

It follows immediately from Lemmas A.2 and A.3 that $\mathcal{H o m}_{\mathcal{B}}\left(B, B^{\prime}\right) \in \operatorname{Set}^{\Delta_{* *}}$. The first four diagrams above ensure that $*$ defines a map

$$
\mathcal{H o m}_{\mathcal{B}}\left(B^{\prime}, B^{\prime \prime}\right) \otimes \mathcal{H o m}_{\mathcal{B}}\left(B, B^{\prime}\right) \rightarrow \mathcal{H}^{\prime} m_{\mathcal{B}}(B, B ")
$$

in $\operatorname{Set}^{\Delta_{* *}}$. The unit rules then ensure that $\zeta^{0} \circ \xi_{0}$ is the multiplicative identity, and the associativity rules make this product associative. 


\section{Appendix B. $A_{\infty}$-algebras and homotopy operads}

$A_{\infty}$-algebras are designed to model deformation retracts of differential graded associative algebras without unit (DGAAs). They are thus an alternative candidate for the task performed in general by s.h. algebras, and are indeed often known as strong homotopy associative algebras (SHAAs). That both concepts are essentially equivalent seems to be folklore (though, if necessary, it could be inferred from the results of Section 6.2).

Now, a DGAA is just a semigroup in the category of cochain complexes (i.e. it satisfies all the requirements of a monoid, except the unit axiom). A third candidate to model deformation retracts of DGAAs is therefore a homotopy semigroup in cochain complexes (defined analogously to a homotopy monoid). These were studied in [Lei1], where it was conjectured that they give rise to $A_{\infty}$-algebras.

\section{B.1. Homotopy semigroups and semicogroups}

Definition B.1. Define $\Delta_{* *}^{+}$to be the subcategory of $\Delta_{* *}$ on objects $\mathbf{n} \neq \mathbf{0}$ and containing only injective morphisms.

Definition B.2. Define a semigroupal category to be a category $\mathcal{C}$ equipped with a bifunctor $\mathcal{C} \times \mathcal{C} \stackrel{\otimes}{\longrightarrow} \mathcal{C}$ satisfying the axioms of a monoidal category (but without a unit object).

Definition B.3. Given a semigroupal category $\mathcal{C}$ (in particular if $\mathcal{C}$ is monoidal), define a quasi-semigroup $X$ in $\mathcal{C}$ to be a colax semigroupal functor $X:\left(\Delta_{* *}^{+}\right)^{\text {opp }} \rightarrow \mathcal{C}$. This means that we have maps

$$
\xi_{m n}: X_{m+n} \rightarrow X_{m} \otimes X_{n},
$$

satisfying naturality and coherence. If $\mathcal{C}$ is a model category, then we say that $X$ is a homotopy semigroup whenever the maps $\xi_{m n}$ are all weak equivalences.

Lemma B.4. Giving a quasi-semigroup $X$ in $\mathcal{C}$ is equivalent to giving objects $X_{n} \in \mathcal{C}$ for $n \in \mathbb{N}_{1}$, together with morphisms

$$
\partial_{i}: X_{n+1} \rightarrow X_{n} \quad 1 \leqslant i \leqslant n,
$$

and an associative coproduct $\xi_{m n}: X_{m+n} \rightarrow X_{m} \otimes X_{n}$, satisfying:

1. $\partial_{i} \partial_{j}=\partial_{j-1} \partial_{i} \quad i<j$.

2. $\left(\partial_{i} \otimes \mathrm{id}\right) \xi_{m+1, n}=\xi_{m n} \partial_{i}$.

3. $\left(\mathrm{id} \otimes \partial_{i}\right) \xi_{m, n+1}=\xi_{m n} \partial_{i+m}$.

Definition B.5. Given a semigroupal category $\mathcal{C}$, define a quasi-semicogroup $X$ in $\mathcal{C}$ to be a lax semigroupal functor $X: \Delta_{* *}^{+} \rightarrow \mathcal{C}$. This means that we have maps

$$
\zeta^{m n}: X^{m} \otimes X^{n} \rightarrow X^{m+n}
$$

satisfying naturality and coherence. If $\mathcal{C}$ is a model category, we say that $X$ is a homotopy semicogroup whenever the maps $\zeta^{m n}$ are all weak equivalences. 
Lemma B.6. Giving a quasi-semicogroup $X$ in $\mathcal{C}$ is equivalent to giving objects $X^{n} \in \mathcal{C}$ for $n \in \mathbb{N}_{1}$, together with morphisms

$$
\partial^{i}: X^{n} \rightarrow X^{n+1} \quad 1 \leqslant i \leqslant n
$$

an associative product $\zeta^{m n}: X^{m} \otimes X^{n} \rightarrow X^{m+n}$, satisfying:

1. $\partial^{j} \partial^{i}=\partial^{i} \partial^{j-1} \quad i<j$.

2. $\zeta^{m+1, n}\left(\partial^{i} \otimes \mathrm{id}\right)=\partial^{i} \zeta^{m n}$.

3. $\zeta^{m, n+1}\left(\mathrm{id} \otimes \partial^{i}\right)=\partial^{i+m} \zeta^{m n}$.

\section{B.2. Homotopy semigroups in abelian categories}

Definition B.7. Given a quasi-semigroup $V$ in an abelian semigroupal category $\mathcal{V}$, define the chain complex $C(V) \in \mathrm{Ch}(\mathcal{V})$ by

$$
C(V)_{n}:= \begin{cases}V_{n} & n>0 \\ 0 & n \leqslant 0\end{cases}
$$

with differential $d=\sum_{i}(-1)^{i} \partial_{i}$.

Definition B.8. A coalgebra $C$ is said to be conilpotent if the iterated coproduct $\Delta^{n}: C \rightarrow C^{\otimes n}$ is 0 for $n$ sufficiently large. A coalgebra $C$ is said to be ind-conilpotent if it is a filtered colimit of conilpotent coalgebras.

Lemma B.9. $C(V)$ has the natural structure of an ind-conilpotent coassociative coalgebra without counit in $\mathrm{Ch}(\mathcal{V})$.

Proof. We define the coproduct $\Delta: C(V) \rightarrow C(V) \otimes C(V)$ by

$$
\bigoplus_{i+j=n} \xi_{i j}: C(V)_{n} \rightarrow(C(V) \otimes C(V))_{n} .
$$

It is straightforward to verify that this is coassociative and a chain map, so $C(V)$ is a coassociative coalgebra without counit.

Observe that the brutal truncations $\sigma_{\leqslant m} C(V)$ of $C(V)$ form conilpotent subcoalgebras of $C(V)$, since $\left(C(V)^{\otimes n}\right)_{i}=0$ for all $i<n$. Thus $C(V)$ is ind-conilpotent, since $C(V)=\lim _{\lessgtr} \sigma_{\leqslant m} C(V)$.

\section{B.2.1. DG coalgebras}

Now assume that $\mathcal{V}$ is the category of cochain complexes of vector spaces over a field $k$. If $V$ is a quasi-semigroup in $\mathcal{V}$, then Lemma B.9 implies that the cochain complex $\operatorname{Tot} C(V)$ (given by $(\operatorname{Tot} C(V))^{n}:=\bigoplus_{i} C(V)_{i}^{i+n}$ ) is an ind-conilpotent coassociative DG coalgebra without counit over $k$.

Definition B.10. Let $D G \mathcal{C}_{k}$ be the category of all ind-conilpotent coassociative DG coalgebras without counit over $k$. Let $D G \mathcal{A}_{k}$ be the category of associative DG algebras without unit over $k$. 
Definition B.11. Define the cobar functor $\beta^{*}: D G \mathcal{C}_{k} \rightarrow D G \mathcal{A}_{k}$ by letting $\beta^{*} C$ be the free graded associative $k$-algebra $\bigoplus_{n>0} C[-1]^{\otimes n}$ on generators $C[-1]$, with differential defined on generators by $d_{C}+\Delta$, for

$$
\Delta: C[-1] \rightarrow(C \otimes C)[-1]=(C[-1] \otimes C[-1])[1]
$$

the comultiplication.

It has right adjoint $\beta: D G \mathcal{A}_{k} \rightarrow D G \mathcal{C}_{k}$ given by letting $\beta A$ be the cofree graded coassociative ind-conilpotent $k$-algebra $\bigoplus_{n>0} A[1]^{\otimes n}$ on cogenerators $A[1]$, with differential defined on cogenerators by $d_{A}+m: A \oplus(A \otimes A)[1] \rightarrow A[1]$, for $m: A \otimes A \rightarrow A$ the multiplication.

Definition B.12. Define the tangent space $\tan C$ of $C \in D G \mathcal{C}_{k}$ to be

$$
\operatorname{ker}(\Delta: C \rightarrow C \otimes C) .
$$

Proposition B.13. There is a cofibrantly generated model structure on $D G \mathcal{C}_{k}$, for which a morphism $f$ is

1. a cofibration if it is injective;

2. a weak equivalence if either of the following equivalent conditions holds:

(a) $\beta^{*} f$ is a quasi-isomorphism.

(b) $f$ can be expressed as a filtered colimit of quasi-isomorphisms $f_{\alpha}: C_{\alpha} \rightarrow D_{\alpha}$ between finite-dimensional objects of $D G \mathcal{C}_{k}$ (note that this is a stronger than requiring that $f$ be a quasi-isomorphism);

3. a fibration if $f$ is cofree as a morphism of ind-conilpotent coassociative graded coalgebras without counit.

Moreover, for a fibrant object $C$ there is a natural isomorphism

$$
\mathrm{H}^{n}(\tan C) \cong \mathrm{H}^{n+1}\left(\beta^{*} C\right) .
$$

With respect to this model structure, $\beta^{*}$ is a left Quillen equivalence, when $D G \mathcal{A}_{k}$ is given its standard model structure.

Proof. Existence of such a model structure is given in [Pri7, Proposition 4.36] for the analogous case of cocommutative coassociative coalgebras and Lie algebras, but the proof carries over to any Koszul-dual pair of quadratic operads, so it adapts to our context (coassociative coalgebras and associative algebras). The generating cofibrations are injective morphisms $f: C \rightarrow D$ between finite-dimensional objects, satisfying the additional property that the coproduct coker $f \rightarrow D \otimes \operatorname{coker} f$ is zero. Generating trivial cofibrations have the additional property that $\mathrm{H}^{*}(\operatorname{coker} f)=0$.

Characterisation of the weak equivalences follows from [Pri7, Proposition 4.42]. That $\beta^{*}$ is a Quillen equivalence follows from [Pri7, Theorem 4.55].

Remark B.14. Note that fibrant objects of $D G \mathcal{C}_{k}$ are those whose underlying coalgebras are cofree. These are precisely strong homotopy associative algebras (SHAAs), as in [Kon], and weak equivalences between these are tangent quasi-isomorphisms. A choice of cogenerators on an SHAA is precisely an $A_{\infty}$-algebra. This means that every $A_{\infty}$-algebra has a weakly equivalent DG associative algebra. 
Corollary B.15. There is a canonical equivalence class of $A_{\infty}$-algebras associated to any quasi-semigroup $V$ in the category of cochain complexes over $k$.

Proof. The $A_{\infty}$-algebra is just a choice of cogenerators on a fibrant replacement for $\operatorname{Tot} C(V)$.

When $V$ is a homotopy semigroup, we wish to relate this $A_{\infty}$-algebra to $V$.

Proposition B.16. Assume that $C \in D G \mathcal{C}_{k}$ is equipped with an exhaustive increasing filtration $0=F_{0} C \subset F_{1} C \subset \cdots$, comultiplicative in the sense that

$$
\Delta\left(F_{n}\right) \subset \sum_{i+j=n} F_{i} \otimes F_{j} \subset C \otimes C,
$$

and for which the resulting maps $\Delta_{n}: \operatorname{gr}_{n}^{F} C \rightarrow\left(F_{1} C\right)^{\otimes n}$ are quasi-isomorphisms. Then there are canonical isomorphisms $\mathrm{H}^{n+1}\left(\beta^{*} C\right) \cong \mathrm{H}^{n}\left(F_{1} C\right)$.

Proof. The filtration $F_{n} C$ induces a filtration on $\beta^{*} C$ by

$$
F_{p}\left(C[-1]^{\otimes n}\right):=\sum_{p_{1}+\cdots+p_{n}=p}\left(F_{p_{1}} C[-1]\right) \otimes\left(F_{p_{2}} C[-1]\right) \otimes \cdots \otimes\left(F_{p_{n}} C[-1]\right) .
$$

Since this filtration is exhaustive and bounded below, the associated spectral sequence

$$
E_{0}^{p q}(F)=\left(\operatorname{gr}_{-p}^{F} \beta^{*} C\right)^{p+q} \Longrightarrow \mathrm{H}^{p+q}\left(\beta^{*} C\right)
$$

converges.

Now, note that $\operatorname{gr}^{F} \beta^{*} C \cong \beta^{*}\left(\operatorname{gr}^{F} C\right)$, and that the quasi-isomorphisms $\Delta_{n}: \operatorname{gr}_{n}^{F} C \rightarrow$ $\left(F_{1} C\right)^{\otimes n}$ induce a graded quasi-isomorphism

$$
\delta: \operatorname{gr}^{F} C \rightarrow \bigoplus_{n>0}\left(F_{1} C\right)^{\otimes n}
$$

in $D G \mathcal{C}_{k}$.

We now define a filtration $W$ on $\beta^{*} C$ by $W^{m} \beta^{*} C=\bigoplus_{n \geqslant m} C[-1]^{\otimes n}$, noting that $F_{p}\left(\beta^{*} C\right) \cap W^{p+1}\left(\beta^{*} C\right)=0$, since $F_{n-1}\left(C^{\otimes n}\right)=0$. Thus $W$ induces a finite filtration on $\operatorname{gr}_{-p}^{F} \beta^{*} C$, so the associated spectral sequence

$$
E_{0}^{n q}(W)=\left(\operatorname{gr}_{W}^{n} \operatorname{gr}_{-p}^{F} \beta^{*} C\right)^{n+q} \Longrightarrow \mathrm{H}^{p+q}\left(\mathrm{gr}_{-p}^{F} \beta^{*} C\right)
$$

converges. Now, the left-hand side is just $\operatorname{gr}_{-p}^{F}\left(C[-1]^{\otimes n}\right)$, so

$$
E_{1}^{n q}(W) \cong \mathrm{H}^{n+q}\left(\operatorname{gr}_{-p}^{F}\left(C[-1]^{\otimes n}\right)\right) .
$$

Therefore $\delta$ induces an isomorphism of $E_{1}$ spectral sequences, making

$$
\operatorname{gr}_{-p}^{F} \beta^{*}(\delta): \operatorname{gr}_{-p}^{F} \beta^{*} C \rightarrow \operatorname{gr}_{-p}^{F} \beta^{*}\left(\bigoplus_{n>0}\left(F_{1} C\right)^{\otimes n}\right)
$$

a quasi-isomorphism. The right-hand side is just $\operatorname{gr}_{-p}^{F} \beta^{*} \beta\left(F_{1} C[-1]\right)$, where $F_{1} C[-1]$ 
is regarded as an object of $D G \mathcal{A}_{k}$ with zero multiplication. Therefore

$$
E_{1}^{p q}(F)=\mathrm{H}^{p+q}\left(\mathrm{gr}_{-p}^{F} \beta^{*} C\right) \cong \mathrm{H}^{p+q}\left(\operatorname{gr}_{-p}^{F} \beta^{*} \beta\left(F_{1} C[-1]\right)\right) .
$$

Now, since $\beta^{*} \dashv \beta$ are a pair of Quillen equivalences, the map

$$
\beta^{*} \beta\left(F_{1} C[-1]\right) \rightarrow F_{1} C[-1]
$$

is a quasi-isomorphism, so

$$
E_{1}^{p q}(F) \cong \begin{cases}\mathrm{H}^{p+q}\left(F_{1} C[-1]\right) & p=-1, \\ 0 & \text { otherwise }\end{cases}
$$

and therefore the spectral sequence collapses at $E_{1}$, giving

$$
\mathrm{H}^{q-1}\left(\beta^{*} C\right) \cong \mathrm{H}^{q-1}\left(F_{1} C[-1]\right)=\mathrm{H}^{q-2}\left(F_{1} C\right)
$$

as required.

The following result confirms a conjecture from [Lei1, §3.5], although not with the proof envisaged there.

Proposition B.17. If $V$ is a homotopy semigroup, then we may choose a representative $A_{\infty}$-algebra with underlying cochain complex $V_{1}$.

Proof. We adapt the proof of [Pri3, Lemma 1.16]. Take a trivial cofibration Tot $C(V) \rightarrow E$, with $E$ fibrant. The filtration $F_{n} C:=\bigoplus_{i \leqslant n} V_{n}$ of $C(V)$ satisfies the conditions of Proposition B.16, since the maps $V_{n} \rightarrow\left(V_{1}\right)^{\otimes n}$ are quasi-isomorphisms by hypothesis. Thus the map $V_{1} \rightarrow \tan E$ is a quasi-isomorphism. Let the quotient be $Q$, and note that this is a contractible cochain complex.

Let $B$ be the cofree coalgebra on generators $Q$; this is trivially fibrant. Since the morphism $\tan E \rightarrow E$ is a cofibration in $D G \mathcal{C}_{k}$, the composite map $\tan E \rightarrow Q \rightarrow$ $B$ must extend to a morphism $f: E \rightarrow B$ in $D G \mathcal{C}_{k}$. Let $A$ be the equaliser of $f$ and the zero map; this is again a cofree object in $D G \mathcal{C}_{k}$, hence fibrant, and $\tan A=$ $\operatorname{ker}(\tan E \rightarrow Q) \cong V_{1}$.

Therefore $A$ is an SHAA with cogenerating space $V_{1}$, so defines an $A_{\infty}$-structure on $V_{1}$.

\section{B.3. DG co-operads}

We now show that the relation between $A_{\infty}$-algebras and homotopy semigroups of cochain complexes has an analogue for operads. Roughly speaking, we will show that a homotopy monad (in the sense of Appendix A) with suitable operadic structure is related to the homotopy operads of [vdL].

Definition B.18. Given an additive cocomplete monoidal category $\mathcal{C}$, we now define a full subcategory $\mathcal{E}(\mathcal{C})$ of the category $\operatorname{End}(\mathcal{C})$ of endofunctors on $\mathcal{C}$. Objects of $\mathcal{E}$ correspond to collections $\left\{P_{n}\right\}_{n \geqslant 0}$, with $P_{n}$ a $\mathcal{C}$-representation of the symmetric group $S_{n}$, with the associated endofunctor given by

$$
V \mapsto \bigoplus_{n} P_{n} \otimes^{S_{n}} V^{\otimes n}
$$

$\mathcal{E}$ forms a monoidal category under composition of functors, and an additive category under $\oplus$. 
Definition B.19. An operad (resp. pseudo operad) on $\mathcal{C}$ is a monoid (resp. semigroup) in $\mathcal{E}(\mathcal{C})$, and a co-operad (resp. pseudo co-operad) on $\mathcal{C}$ is a comonoid (resp. semicogroup) in $\mathcal{E}(\mathcal{C})$.

Note that since $\mathcal{E}(\mathcal{C})$ is an additive category, there is a natural retraction

$$
(F \circ X) \oplus(F \circ Y) \rightarrow F \circ(X \oplus Y) \rightarrow(F \circ X) \oplus(F \circ Y),
$$

for any $F, X, Y \in \mathcal{E}(\mathcal{C})$. Thus, augmented operads $\top$ on abelian categories $\mathcal{C}$ correspond to pseudo operads $S$ on $\mathcal{C}$, by setting $S:=\operatorname{ker}(\top \rightarrow 1)$ and $\top=S \oplus 1$.

Let $d g$ Vect be the category of chain complexes, and $g$ Vect the category of graded vector spaces, both over a field of characteristic 0 .

Lemma B.20. If $\mathcal{C}=d g$ Vect or $g$ Vect, then the forgetful functor from pseudo operads on $\mathcal{C}$ to $\mathcal{E}(\mathcal{C})$ has a left adjoint, denoted by $T$. Likewise, the forgetful functor from indconilpotent pseudo co-operads on $\mathcal{C}$ to $\mathcal{E}(\mathcal{C})$ has a right adjoint, denoted by $T^{\prime}$.

Proof. These are described in [vdL, §2]. In both cases, the underlying functor is

$$
F \mapsto \bigoplus_{n>0} \overbrace{F \circ F \circ \cdots \circ F}^{n} .
$$

Definition B.21. Recall from [vdL, Definition 3.1] that an operad up to homotopy on $d g$ Vect is defined to be a collection $P \in \mathcal{E}(g$ Vect $)$, together with a square-zero differential $\delta$ on the cofree pseudo co-operad $T^{\prime}(P[1])$.

The notion of operad up to homotopy in [ $\mathbf{v d L}]$ generalises pseudo operads (or, equivalently, augmented operads) rather than operads. This motivates the following comparison, noting that the quasi-monads of Appendix A are a fortiori quasi-semigroups in $\operatorname{End}(\mathcal{C})$, and that pseudo co-operads can be replaced by homotopy operads, similarly to Corollary B.15.

Lemma B.22. Every quasi-semigroup $Q$ in $\mathcal{E}(d g$ Vect) (in the sense of Definition B.3) naturally gives rise to a pseudo co-operad $\beta(Q)$ on $d g$ Vect.

Proof. Given a quasi-semigroup $Q=\left\{Q_{n}\right\}_{n>0}$ in $\mathcal{E}(d g$ Vect), we may set $\beta(Q):=$ $\bigoplus_{n>0} Q_{n}[n]$ in $\mathcal{E}(g$ Vect $)$, with differential $\delta:=d_{Q} \pm \sum_{i}(-1)^{i} \partial_{i}$. Here, $\partial_{i}: Q_{n}[n]_{j} \rightarrow$ $Q_{n+1}[n+1]_{j-1}$ is the structural map $\partial_{i}:\left(Q_{n}\right)_{n+j} \rightarrow\left(Q_{n+1}\right)_{n+j}$ of the quasi-semigroup.

The functor $\beta(Q)$ has the natural structure of a pseudo co-operad, with coproduct $\beta(Q) \rightarrow \beta(Q) \circ \beta(Q)$ given on $Q_{n}[n] \subset \beta(Q)$ by $\sum_{i+j=n} \xi_{i j}: Q_{n}[n] \rightarrow Q_{i}[i] \circ Q_{j}[j]$, making use of the natural retraction $(F \circ X) \oplus(F \circ Y) \rightarrow F \circ(X \oplus Y) \rightarrow(F \circ X) \oplus$ $(F \circ Y)$.

\section{References}

[Ber1] J. E. Bergner, A model category structure on the category of simplicial categories, Trans. Amer. Math. Soc. 359 (2007), no. 5, 2043-2058 (electronic).

[Ber2] J. E. Bergner, Three models for the homotopy theory of homotopy theories, Topology 46 (2007), no. 4, 397-436. 
[BJ] T. P. Bisson and A. Joyal, $Q$-rings and the homology of the symmetric groups, in Operads: Proceedings of Renaissance Conferences (Hartford, CT/Luminy, 1995), Contemp. Math. 202, 235-286, Amer. Math. Soc., Providence, RI, 1997.

[CLM] F. R. Cohen, T. J. Lada and J. P. May, The homology of iterated loop spaces, Lecture Notes in Mathematics 533, Springer-Verlag, New York, 1976.

[DV] M. Dubois-Violette. Dérivations et calcul différentiel non commutatif, C.R. Acad. Sci. Paris Sér. I Math. 307 (1988), no. 8, 403-408.

[GJ] P. G. Goerss and J. F. Jardine, Simplicial homotopy theory, Progress in Mathematics 174, Birkhäuser Verlag, Basel, 1999.

[Hin] V. Hinich, DG coalgebras as formal stacks, J. Pure Appl. Algebra 162 (2001), no. 2-3, 209-250.

[Hir] P. S. Hirschhorn, Model categories and their localizations, Mathematical Surveys and Monographs 99, Amer. Math. Soc., Providence, RI, 2003.

[Hov] M. Hovey, Model categories, Mathematical Surveys and Monographs 63, Amer. Math. Soc., Providence, RI, 1999.

[JT] A. Joyal and M. Tierney, Quasi-categories vs Segal spaces, in Categories in algebra, geometry and mathematical physics, Contemp. Math. 431, 277-326, Amer. Math. Soc., Providence, RI, 2007.

[Kon] M. Kontsevich, Topics in algebra - deformation theory, Lecture Notes, available at http://www.math. brown.edu/ abrmovic/kontsdef .ps, 1994.

[KS] M. Kontsevich and Y. Soibelman, Deformations of algebras over operads and the Deligne conjecture, in Conférence Moshé (Flato 1999, Vol. I, Dijon), Math. Phys. Stud. 21, 255-307, Kluwer Acad. Publ., Dordrecht, 2000.

[Lei1] T. Leinster, Homotopy algebras for operads, arXiv:math/0002180v1 [math.QA], 2000.

[Lei2] T. Leinster, Up-to-homotopy monoids, arXiv:math.QA/9912084v2, 2000.

[Lur] J. Lurie, Derived algebraic geometry, Ph.D. thesis, M.I.T., 2004, http:// hdl.handle.net/1721.1/30144.

[Mac] S. MacLane, Categories for the working mathematician, Graduate Texts in Mathematics 4, Springer-Verlag, New York, 1971.

[Man1] M. Manetti, Deformation theory via differential graded Lie algebras, in Algebraic Geometry Seminars, 1998-1999 (Italian) (Pisa), pp. 21-48, Scuola Norm. Sup., Pisa, 1999.

[Man2] M. Manetti, Extended deformation functors, Int. Math. Res. Not. 14 (2002), 719-756.

[Pri1] J. P. Pridham, Deforming $l$-adic representations of the fundamental group of a smooth variety, J. Algebraic Geom. 15 (2006), no. 3, 415-442.

[Pri2] J. P. Pridham, Deformations of schemes and other bialgebraic structures, Trans. Amer. Math. Soc. 360 (2008), no. 3, 1601-1629.

[Pri3] J. P. Pridham, Pro-algebraic homotopy types, Proc. London Math. Soc. 97 (2008), no. 2, 273-338, 
[Pri4] J. P. Pridham, Derived deformations of Artin stacks, arXiv:0805.3130v2 [math.AG], 2009.

[Pri5] J. P. Pridham. Derived deformations of schemes, arXiv:0908.1963v1 [math.AG], 2009.

[Pri6] J. P. Pridham, Constructing derived moduli stacks, in preparation, 2010.

[Pri7] J. P. Pridham, Unifying derived deformation theories, Adv. Math. 224 (2010), no. 3, 772-826,

[PW] J. Power and H. Watanabe, Combining a monad and a comonad, in Coalgebraic methods in computer science (Amsterdam, 1999), Theoret. Comput. Sci. 280 (2002), no. 1-2, 137-162,

[Qui1] D. Quillen, Rational homotopy theory, Ann. of Math. (2) 90 (1969), no. 2, 205-295.

[Qui2] D. G. Quillen, Homotopical algebra, Lecture Notes in Mathematics 43, Springer-Verlag, New York, 1967.

[Rez] C. Rezk, A model for the homotopy theory of homotopy theory, Trans. Amer. Math. Soc. 353 (2001), no. 3, 973-1007 (electronic).

[vdL] P. van der Laan, Operads up to homotopy and deformations of operad maps, arXiv:math/0208041v1 [math.QA], 2002.

[VO] D. H. Van Osdol, Bicohomology theory, Trans. Amer. Math. Soc. 183 (1973), 449-476.

[Wei] C. A. Weibel, An introduction to homological algebra, Cambridge University Press, Cambridge, 1994.

\section{J. P. Pridham J.P.Pridham@dpmms.cam.ac.uk}

Dept. of Pure Mathematics and Mathematical Statistics, Centre for Mathematical Sciences, University of Cambridge, Wilberforce Road, Cambridge CB3 0WB, U.K. 